\title{
Les formations interdisciplinaires : problèmes, expériences, perspectives
}

\author{
Journées de l'Association \\ Natures Sciences Sociétés Dialogues, \\ Paris, ENS \\ 7 et 8 février 2007
}




\title{
SOMMAIRE
}

\author{
PRESENTATION \\ Marcel JOLLIVET, sociologue, CNRS
}

COMPTE-RENDU DES JOURNEES

Marcel JOLLIVET, sociologue, CNRS

$\begin{array}{lr}\text { INTERVENTIONS CHOISIES } & 15\end{array}$

Disciplines et enseignements pluridisciplinaires $r$ Henri BUC, Professeur honoraire à I'Institut Pasteur

Le master Communication scientifique et technique : contenus, outils, pratiques Jean-Louis MARTINAND, Professeur de didactique des sciences et techniques, École normale supérieure de Cachan

Commentaires sur le compte rendu Jean-Claude MOUNOLOU, Professeur honoraire des universités, biologiste Muriel MAILLEFERT, MCF, université de Lille 3, économiste

TEMOIGNAGE DES JEUNES DIPLOMES

Livio RIBOLI-SASCO, École normale supérieure et Elifsu SABUNCU, Institut Pasteur

UN PANEL DE FORMATIONS INTERDISCIPLINAIRES

1. Sciences de I'univers, environnement, écologie (Luc ABBADIE)

2. Logique, philosophie, histoire, sociologie des sciences (LoPHiSS Paris-Sorbonne)

(Daniel ANDLER)

3. Développement et aménagement intégré des territoires (DAIT) (Patrick BLANDIN)

4. Développement durable, management environnemental et géomatique (Catherine CARRE)

5. MASS - Modélisation et gestion sociale des risques (Cécilia CLAEYS-MEKDADE)

6. Environnement, société : dynamiques et interfaces (Daniel DELAHAYE)

7. Cogmaster (Emmanuel DUPOUX)

8. Vie, Terre, Environnement, Santé, Société (VTESS) (Patrick GIRAUDOUX)

9. Espaces, sociétés rurales et logiques économiques (ESSOR) (Anne-Marie GRANIE)

10. Ingénierie et management de l'environnement et du développement durable (IMEDD) (Bertrand GUILLAUME)

11. Économie et gestion de l'environnement et du développement durable (Muriel MAILLEFERT) 57

12. Archéologie et histoire (Dominique MARGUERIE)

13. Didactique des sciences et techniques (Jean-Louis MARTINAND)

14. Écologie humaine : enjeux environnementaux des activités de production et de consommation (Francis RIBEYRE) 
15. Certificat international d'écologie humaine (CIEH) (Francis RIBEYRE)

16. Environnement : milieux, techniques, sociétés (EMTS) (Marie ROUE)

17. Modèles et systèmes en biologie : approches interdisciplinaires du vivant (AIV) (François TADDEİ, Livio RIBOLI-SASCO)

18. Une maîtrise en sciences de I'environnement à I'université du Québec, Montréal (Laurent LEPAGE)

1. Argumentaire et programme des Journées

2. Textes sur la formation dans la revue Natures Sciences Sociétés 


\section{Présentation}

Marcel JOLLIVET, sociologue, CNRS

L'idée d'une formation impliquant l'apprentissage conjoint de plusieurs disciplines ne date pas d'aujourd'hui ${ }^{1}$. Elle est monnaie courante dans les écoles d'ingénieurs (sans parler de la médecine). Elle peut même être consubstantielle à certaines disciplines universitaires anciennes (comme la géographie ou l'archéologie, par exemple).

Toutefois, les expériences de formation combinant plusieurs disciplines connaissent aujourd'hui un regain d'intérêt. Elles se multiplient dans les universités. Elles s'étendent à de nouveaux domaines (notamment aux thèmes en rapport avec la question de l'environnement et, plus récemment, du développement durable). Les spectres disciplinaires qu'elles recouvrent tendent à s'élargir (en particulier aux sciences sociales). L'idée d'une formation à caractère interdisciplinaire fait même l'objet d'un débat récurrent à propos des enseignements primaire et secondaire.

Or, un quadruple constat peut être fait en la matière.

Le premier part d'une évaluation de la production scientifique à caractère interdisciplinaire. De ce point de vue, la leçon à tirer de plus de dix années d'existence de la revue Natures Sciences Sociétés est sans équivoque : la formation à l'interdisciplinarité est une condition essentielle - et, dans l'état actuel des choses, un goulet d'étranglement - pour le développement d'une production scientifique réellement interdisciplinaire (au moins dans les domaines couverts par la revue). Tant qu'elle n'aura pas été l'objet des réflexions qu'elle exige, la pratique de la recherche interdisciplinaire dans ces domaines stagnera au point de risquer de compromettre sa crédibilité scientifique. De ce point de vue, un préalable à lever se situe au niveau de la formation.

Le deuxième est celui que l'on peut tirer d'un tour d'horizon des formations universitaires du niveau du master : même rapide et superficiel, un tel tour d'horizon montre que nombreuses sont celles qui se réclament dans leurs intitulés de l'interdisciplinarité. On peut donc parler d'un réel mouvement en ce sens.

Le troisième prolonge le second : c'est la diversité et la dispersion des initiatives. Les contextes institutionnels dans lesquels elles se déploient, les thématiques sur lesquelles elles se construisent, les objectifs qu'elles poursuivent sont très divers. Chacune a, de ce fait, des caractéristiques qui lui sont propres. Cette diversité est une richesse. Encore faut-il que les conditions soient réunies pour l'exploiter. Or, les expériences faites sont très dispersées, elles n'ont aucune visibilité collective. Loin de donner lieu à une réflexion partagée, elles tendent à se clore sur elles-mêmes. Cela vient de ce que les mettre en place et les faire vivre dans un contexte qui ne leur facilite guère l'existence exige un fort investissement en énergie et en temps, lequel s'ajoute à celui que requièrent par ailleurs les activités d'enseignement plus classiques. L'investissement nécessaire est d'autant plus lourd qu'elles supposent des innovations qui ont souvent, du fait même de leur caractère novateur, du mal à se construire, tant sur le plan de leur contenu (en termes de connaissances à réunir) que sur celui de leurs démarches de formation (la structure de leur enseignement et leur pédagogie) et sur celui de leur organisation institutionnelle.

Le dernier est la conclusion à laquelle conduit une lecture attentive des réflexions que suscite l'interdisciplinarité. Le fait frappant est leur convergence. En effet, ce sont toujours les

\footnotetext{
${ }^{1}$ Ce supplément au n²/2008 de Natures Sciences Sociétés rend compte des Journées 2007 de l'association « Natures Sciences Sociétés Dialogues » qui se sont déroulées sous ce titre les 7 et 8 février 2007 dans les locaux de l'École normale supérieure, rue d'Ulm à Paris.
} 
mêmes questions qui sont soulevées lorsque l'on veut la mettre en pratique de façon réelle et rigoureuse. Et ceci quel que soit le domaine concerné. Ainsi, contrairement à ce qu'en pensent ceux qui les assument, pris qu'ils sont dans les difficultés de son montage, on peut dire que les problèmes que pose chaque expérience particulière ne résultent pas simplement des contingences liées à la situation locale à laquelle ils ont à faire face. Au-delà ou derrière celles-ci, il y a bien un socle commun de questions de fond. On est bien face à une problématique scientifique de portée générale.

Or, celle-ci n'est nulle part traitée en tant que telle. Elle n'est même pas identifiée en tant que question scientifique. C'est là une entrave au développement d'une interdisciplinarité de qualité. C'est la raison pour laquelle l'association Natures Sciences Sociétés Dialogues a décidé d'organiser les Journées qui donnent lieu à ce supplément de la revue Natures Sciences Sociétés. Identifier ensemble les problèmes communs, partager les interrogations, mais aussi les pistes tentées par les uns et les autres pour y répondre sont devenus des nécessités pour passer à une autre étape. L'expérience acquise permet désormais qu'on tente d'en partager la richesse. Cette réflexion devrait permettre de mieux faire face aux difficultés que les initiatives rencontrent, la plupart du temps, pour trouver place dans les structures de formation existantes. Elle devrait aussi contribuer, sur un plan général, à réfléchir sur les bases épistémologiques des démarches interdisciplinaires. D'où les orientations proposées à la réflexion des participants.

L'idée générale est que les enseignants qui se lancent dans la conception et la mise en œuvre d'une formation interdisciplinaire ne le font pas sans de fortes raisons compte tenu de la charge de travail que cela représente ${ }^{2}$. Celles-ci peuvent être d'ordre divers : finalités de la formation (notamment pour les formations professionnelles), finalités institutionnelles, intérêt intellectuel, orientation scientifique, etc. Cela suppose l'explicitation et l'élaboration d'un projet de formation original, conçu en fonction de sa raison d'être, et qui doit donc répondre à ses exigences. Ce projet est le résultat d'une réflexion nécessairement collective. C'est de cette dernière, et de ses péripéties éventuelles, que doit partir toute analyse de l'expérience en cause. Certaines formations interdisciplinaires peuvent être largement le produit des circonstances et procéder d'un pragmatisme de bon aloi. Il n'en demeure pas moins que, si elles sont tentées, c'est qu'elles correspondent à un projet.

Ce projet s'incarne dans la forme pratique que prend la formation dans les masters qui sont mis en place. Un point à souligner ici est que les choix faits pour cette mise en pratique impliquent, qu'elle ait été l'objet d'une réflexion ou non, une conception de la façon d'associer des disciplines différentes dans un cursus unique. Ces choix sont certes largement fonction des finalités poursuivies et des conjonctures locales, mais ils renvoient aussi à une posture intellectuelle. Une attention toute particulière doit donc être portée à la formule pédagogique adoptée. Les disciplines associées, les méthodologies privilégiées, les modalités de la formation et de l'évaluation retenues etc. sont l'expression du projet de formation envisagé et de la conception de l'interdisciplinarité qui en est le soubassement. C'est en eux que se jouent les enjeux intellectuels de l'interdisciplinarité et que résident les difficultés que rencontre sa mise en œuvre sur le plan scientifique.

De ce point de vue, on peut envisager deux positions extrêmes. Le souci peut simplement être de doter les étudiants d'une culture scientifique reposant sur plusieurs disciplines ; ceci peut passer par un cursus qui se contente de juxtaposer des enseignements disciplinaires donnés dans des formes tout à fait habituelles. À l'extrême opposé, le cursus proposé peut être délibérément construit autour d'une démarche visant à réfléchir - et à faire réfléchir - sur le découpage disciplinaire qui est au cœur du projet de formation et à trouver les voies d'un dialogue entre les disciplines concernées; ceci suppose des modalités de

\footnotetext{
${ }^{2}$ Les lignes qui suivent s'inspirent du texte de l'argumentaire des Journées ( $c f$. Annexe).
} 
formation originales. Dans le premier cas, il pourrait sembler plus approprié de parler de " pluridisciplinarité », plutôt que d' "interdisciplinarité ». Et dans le second cas, on pourrait parler simultanément de «formation interdisciplinaire» et de «formation à l'interdisciplinarité ».

Mais les distinctions à faire sont sans doute plus subtiles. Une polarisation forte des disciplines convoquées autour d'objets, de thèmes et de questions de recherche largement partagés peut produire une interdisciplinarité de fait, sans qu'il soit nécessaire de se donner des moyens de formation particuliers pour la mettre en pratique: elle est alors comme immergée dans chacune des composantes de la formation. Cette construction d'une interdisciplinarité que l'on pourrait qualifier d'implicite est encore renforcée si la polarisation en question est par ailleurs associée à une longue expérience d'enseignement en commun. Ceci n'est sans doute possible qu'entre des disciplines proches sur le plan épistémologique. Dans d'autres cas, la formation sur et par l'interdisciplinarité impliquera une relation plus réflexive entre les disciplines et les enseignants, et donc une véritable initiation à l'épistémologie.

Pour comprendre ce qui se passe au niveau des échanges intellectuels, il peut être utile de reprendre ici la distinction entre « interdisciplinarité de proximité » (c'est-à-dire entre des disciplines d'ores et déjà susceptibles de partager un langage et des démarches de recherche communs) et " interdisciplinarité élargie » (c'est-à-dire entre des disciplines entre lesquelles ce travail de construction d'un langage et de démarches de recherche communs reste à faire). Entrent aussi en ligne de compte les exigences, en termes de croisement des disciplines, de la problématique qui sous-tend la formation. Elles peuvent être plus ou moins fortes. Il est également nécessaire de prendre en compte le poids des contraintes institutionnelles locales et de mettre en évidence les compromis éventuels auxquels elles ont conduit.

Le principe était d'offrir un temps et un espace d'information réciproque et de débats autour des réalisations présentées et le programme était conçu autour d'une double idée ${ }^{3}: 1$ ) prendre appui sur des interdisciplinarités exemplaires, soit nanties d'une expérience ancienne, soit plus récentes mais très actives, pouvant servir de modèle de référence ; 2) donner à voir toute une gamme, la plus diverse et la plus ouverte possible, d'initiatives universitaires novatrices, constituant comme un banc d'essai pour des formations interdisciplinaires prenant en charge des thèmes nouveaux. L'avenir de l'interdisciplinarité passant par une meilleure formation des jeunes chercheurs, une place était donnée à l'expression des étudiants ayant bénéficié des formations présentées.

Trois raisons ont conduit à consacrer un supplément de la revue à ces Journées : la richesse de la matière réunie, son originalité et la conclusion à laquelle elles ont conduit. Il est clair en effet que le travail de réflexion entrepris n'en est qu'à ses débuts. Nombreuses sont les initiatives qui mériteraient tout autant d'attention que les dix-huit qui figurent ici. Les Journées ont davantage permis de commencer une enquête et d'identifier les pistes de réflexion à suivre que d'approfondir les questions soulevées par la formation interdisciplinaire. Ce supplément se veut donc un outil pour poursuivre cet approfondissement en lui fournissant un point de départ.

Ceci explique son contenu : un compte rendu général des débats; des commentaires de participants ; le témoignage d'un groupe d'étudiants et, pour finir, un rappel des présentations des expériences. Ces présentations sont construites sur la base d'une grille commune dont l'objectif central est la façon dont l'interdisciplinarité est mise en œuvre au sein des masters. C'est aussi l'axe majeur du compte rendu et aussi bien la problématique même des Journées ${ }^{4}$.

\footnotetext{
${ }^{3} C f$. en annexe le programme des Journées.

${ }^{4}$ Ce supplément de la revue Natures Sciences Sociétés a été réalisé par Marcel Jollivet et Marie-Alix Carlander.
} 


\section{Compte-rendu des Journées}

Marcel JOLLIVET, sociologue, CNRS

Le fait que l'acquisition des compétences exigées par un profil de formation donné puisse supposer un recours à différents domaines de la connaissance est admis comme allant de soi. Cette exigence est à la base de tout enseignement professionnel. Il en est ainsi en particulier, au niveau de l'enseignement supérieur, de l'enseignement donné dans les écoles d'ingénieurs (et, notamment, dans les Grandes Écoles). De ce point de vue, l'interdisciplinarité - ou, du moins, le mariage de disciplines dans l'enseignement - est une pratique banale. Et, donc, une banalité pratique : allant de soi, elle n'appelle aucune réflexion particulière et, de fait, n'en suscite aucune. Il est probable d'ailleurs qu'il s'agisse davantage de formations pluridisciplinaires que véritablement interdisciplinaires, les différents enseignements étant plus juxtaposés que véritablement intégrés les uns aux autres. C'est aux intéressés qu'il revient, dans leur pratique professionnelle, de procéder aux synthèses et aux compromis cognitifs qu'exigent les tâches qu'ils ont à accomplir.

Il faut toutefois noter que les sciences dites «pour l'ingénieur» ont, par définition une forte composante véritablement interdisciplinaire, ce qui justifierait que l'on parle à leur propos de «sciences de » et non pas de «sciences pour », le «pour» niant précisément le travail d'intégration interdisciplinaire qui est à la base du travail propre de l'ingénieur. On peut citer comme exemples la mécanique, ou l'agronomie, ou la médecine, ou l'architecture etc. ou, pour prendre un exemple plus récent, l'ingénierie écologique. Le refus de prendre acte $\mathrm{du}$ caractère scientifique de ce travail d'intégration interdisciplinaire se traduit par le flottement de la terminologie. En effet, comme ils ont une finalité pratique et ont en conséquence un caractère composite, les champs de connaissance en question sont plus volontiers qualifiés d' «arts » que de disciplines. Comme si le travail de recomposition des connaissances qu'exige la création appliquée, "technique », ne pouvait pas être source de connaissance en soi, et source de connaissance originale du fait même qu'il procède d'une démarche visant à rapprocher ce qui, d'une certaine manière arbitrairement, avait été séparé !

L'intérêt nouveau porté aujourd'hui à l'interdisciplinarité vient précisément de la prise de conscience du fait que le chercheur se trouve, dans son travail de recherche, face à la même complexité que celle à laquelle l'ingénieur est affronté dans son action; et qu'il a donc à s'interroger sur sa façon propre de l'intégrer dans sa propre démarche. Ceci résulte sans doute de deux évolutions convergentes : c'est, en premier lieu, l'avancée des connaissances ellemême qui se traduit par leur spécialisation et leur dispersion croissantes, tout en mettant paradoxalement toujours plus en évidence la complexité des phénomènes; il devient donc de plus en plus difficile de ne pas la prendre en considération. C'est, par ailleurs - même si ce sont là des considérations qui demeurent pour l'instant encore largement méconnues, si ce n'est la plupart du temps récusées par les chercheurs - la proximité croissante entre les connaissances scientifiques et leurs applications, elles-mêmes de plus en plus complexes; ceci rapproche le chercheur de la position de l'ingénieur et lui confère une responsabilité qui s'apparente à celle de ce dernier, comme en témoigne la montée en puissance des préoccupations éthiques dans les activités de recherche. Il est donc devenu indispensable de repenser l'articulation entre les connaissances spécialisées, sous disciplinaires même plus que disciplinaires, et de recomposer des schémas de compréhension généraux, interdisciplinaires, qui permettent de leur redonner la place et le sens tout relatifs qu'elles ont, dans le contexte de la complexité qui est à prendre en considération compte tenu de l'objectif poursuivi. 
L'objectif du colloque était de partir de présentations d'expériences de formation interdisciplinaires pour examiner la façon dont elles prenaient en charge ces interrogations ${ }^{5}$. Pour mener cette réflexion, les témoignages réunis ont été de quatre types ${ }^{6}$ :

- le premier répond au souci de prendre appui sur l'expérience de formations interdisciplinaires anciennes et donc bien rodées, reconnues sur le plan universitaire et institutionnalisées de longue date ; l'exemple de l'archéologie s'impose à l'esprit ; il a paru d'autant plus justifié de la choisir qu'elle illustre une interdisciplinarité très complète, car associant aussi bien des sciences de la nature que des sciences de la société ;

- deux concernent au contraire des domaines de recherche plus ou moins nouveaux, en plein développement, à interdisciplinarité très large également, puisqu'à cheval sur les sciences de la vie et les sciences humaines et sociales (les sciences cognitives et l'écologie humaine) ;

- deux autres encore rendent compte des démarches de formation interdisciplinaire dans les Grandes Écoles : une école d'ingénieurs (l'École nationale du génie rural et des eaux et forêts) et l'École normale supérieure ;

- treize enfin relatent des expériences de masters universitaires ; choix délibéré, la plupart concernent des thèmes en rapport avec l'environnement; leur intérêt est d'illustrer toute une gamme de situations, avec des interdisciplinarités plus ou moins larges et des finalités différentes; il s'agissait, sans aucune prétention à une quelconque représentativité, de se donner une idée qualitative des pratiques actuelles d'enseignement universitaire dans le domaine de l'environnement.

Les observations majeures que l'on peut tirer d'un tour d'horizon de ces dix-huit témoignages peuvent être regroupés autour de trois grandes rubriques : une caractérisation des masters par leurs objectifs, leurs démarches d'ensemble et leurs pratiques pédagogiques ; un état des questions que soulève ce type de formation; une caractérisation générale de l'interdisciplinarité telle qu'elle ressort de ces expériences.

\section{Caractérisation des masters}

Les raisons pour lesquelles l'interdisciplinarité est recherchée sont variables. Dans le cas des sciences cognitives, c'est la constitution d'un champ de recherche qui est en jeu. Dans cette même perspective, l'objectif peut carrément être la constitution d'une discipline : c'est déjà fait pour l'archéologie, qui est reconnue en tant que telle; c'est ce qui est tenté pour l'écologie humaine.

Mais dans la plupart des cas, l'ambition est plus limitée : c'est le thème qui figure dans l'intitulé du master qui commande son organisation. Trois cas de figure peuvent alors se présenter. Il peut s'agir :

- de stimuler une discipline à travers la confrontation interdisciplinaire (ex : la biologie dans « Approches interdisciplinaires du vivant ») ;

- de construire un champ de recherche en interdisciplinarité (ex : « Environnement : milieux, techniques, sociétés $»)$;

- de donner une formation professionnelle supposant l'interdisciplinarité (ex : « Développement durable, management environnemental et géomatique »).

Dans tous les cas, l'objectif de former en vue de débouchés est bien sûr présent, mais avec des visées diverses. Il est clairement orienté vers la recherche dans le premier cas de figure, plutôt orienté vers la recherche dans le deuxième, mais sans exclure d'autres possibilités; et clairement orienté vers des emplois publics ou privés hors $\mathrm{du}$ monde

\footnotetext{
${ }^{5} C f$. en annexe l'argumentaire du colloque.

${ }^{6} \mathrm{Cf}$. en annexe le programme du colloque.
} 
universitaire dans le troisième. Les masters du premier et du deuxième cas de figure ont comme objectif premier d'utiliser la formation par la recherche pour créer un corps de chercheurs capable de prendre en main de façon interdisciplinaire le champ de recherche couvert par le master. Mais, ceux du deuxième cas de figure sont conçus pour donner en même temps aux étudiants une compétence professionnelle susceptible de déboucher sur un emploi non universitaire ; on peut voir dans ce caractère hybride le souci d'avoir des chances de recruter des étudiants. Ceux du troisième cas de figure jouent résolument la carte de la professionnalisation par l'université. On notera que pas moins de cinq des masters sont classés masters purement professionnels. Ce qui n'empêche pas qu'ils puissent former, comme ceux du deuxième cas, de futurs enseignants-chercheurs ou chercheurs. Leurs contenus sont cependant plus techniques. Il apparaît finalement que les masters qui jouent résolument le jeu d'une formation à une recherche interdisciplinaire en soi sont des exceptions; la plupart, au contraire, s'appuient sur une - encore bien embryonnaire naissance d'un marché de l'emploi (en l'occurrence dans le domaine de l'environnement), supposant des compétences interdisciplinaires, pour former d'éventuels jeunes chercheurs ou enseignants-chercheurs ayant un profil interdisciplinaire. Ceux qui en prennent l'initiative soulignent qu'ils se heurtent à la rude concurrence des Grandes Écoles.

Les démarches adoptées pour la formation sont d'une grande diversité. Les formules utilisées vont du cours magistral classique aux travaux personnalisés en passant par des séminaires, l'observation sur terrain, le stage et le voyage d'études. Tous les masters combinent plusieurs de ces formules. Les cours sont de deux sortes: des cours de tronc commun (la plupart des masters en ont un) et des cours d'options (pratiquement tous les masters en ont). Tous les masters donnent une place au travail personnel, généralement validé par un mémoire. Mais cette place est extrêmement variable d'un master à l'autre. Elle est nettement plus importante dans les formations des Grandes Écoles; elle y prend même nettement le pas sur les enseignements magistraux. Dans les masters recherche universitaires, le mémoire est une préfiguration d'une thèse. Dans les masters professionnels, il est davantage un exercice mettant en valeur une compétence acquise. Dans le cadre de ce travail personnel, une importance plus ou moins grande est donnée à un travail collectif entre les étudiants. C'est là un point majeur dans l'organisation pédagogique des masters, car l'échange direct entre des étudiants de profils scientifiques différents est un excellent moyen de promouvoir une véritable interdisciplinarité.

La logique d'ensemble des démarches de formation s'inscrit dans quatre profils: apporter des connaissances nouvelles sur une thématique donnée à une population d'étudiants venant d'horizons très différents, mais intéressés par la même thématique; donner des connaissances dans un ensemble de disciplines considérées comme complémentaires pour le domaine couvert par le master; chercher à produire une greffe entre disciplines, susceptible de déboucher sur une compétence transversale; donner un savoir-faire intégrant des compétences d'ordre divers. Chaque master combine plusieurs de ces quatre ambitions. Mais chacun d'eux les hiérarchise différemment. Les choix faits (ou les arbitrages faits compte tenu $\mathrm{du}$ jeu contradictoire des potentialités et des contraintes locales) s'expriment dans le poids respectif des enseignements magistraux et des travaux personnels, dans le poids respectif du tronc commun et des options, dans le nombre des disciplines associées, dans leur plus ou moins grande proximité sur le plan scientifique. Plus le nombre des disciplines couvertes est grand, plus les enseignements magistraux prennent de l'importance, plus le tronc commun est lourd. On tend alors vers une pluridisciplinarité de base et une multiplication des options, et donc vers la spécialisation des mémoires. Au contraire, cibler la formation sur une fécondation réciproque de disciplines contraint à en réduire le nombre pour pouvoir approfondir les questions que pose leur croisement et de réduire d'autant la place des enseignements, à accorder une part plus importante au travail personnel, au travail en petits 
collectifs... Enfin, vouloir donner un savoir-faire intégrant des connaissances et des outils venant d'horizons variés se traduit par une place plus grande donnée à une mise en pratique.

\section{Les points-clés d'une formation interdisciplinaire}

Deux raisons majeures peuvent expliquer les initiatives prises pour créer des formations interdisciplinaires : 1) la conviction scientifique que l'interdisciplinarité est une voie de recherche en soi à explorer et que c'est de la formation de jeunes chercheurs sachant la pratiquer que dépendra la place qu'elle pourra prendre dans la recherche universitaire (on est là proche parfois du militantisme) ; 2) le sentiment que les universités doivent s'ouvrir à la formation professionnelle et que cela passe par des formations nécessairement interdisciplinaires (là, c'est le principe de réalité qui domine). Ces deux raisons ne sont pas nécessairement exclusives l'une de l'autre, mais elles peuvent avoir pesé d'un poids différent dans la création de tel ou tel master. Une des conditions de la survie de ces formations est d'ailleurs de jouer sur les deux tableaux à la fois, ce qui ne facilite pas la tâche de leurs responsables.

Les initiatives impliquent souvent des enseignants-chercheurs qui ont déjà une expérience de l'interdisciplinarité ${ }^{7}$ et qui sont donc convaincus de son intérêt scientifique. Le sentiment qui domine est qu'il faudrait pouvoir la "penser » avant de l'enseigner et que le temps manque pour le faire. De ce fait, un certain pragmatisme prévaut qui pousse à prouver le mouvement en marchant. Et à apprendre en faisant. La modestie est donc de rigueur. Elle n'empêche pas la prise de risque, notamment auprès des étudiants. D'où l'intérêt de la façon dont ces derniers évaluent la formation qu'ils ont reçue ${ }^{8}$.

Les formations interdisciplinaires ont plusieurs problèmes délicats à résoudre, ceux ayant trait :

- à la place et au rôle à donner au tronc commun, ainsi qu'à l'approche pédagogique qu'il faut en avoir ;

- à l'approche pédagogique des enseignements spécialisés ;

- à l'hétérogénéité des profils des étudiants ;

- à la recherche d'une intégration des connaissances ;

- au rapport à l'action ;

- à l'évaluation des étudiants ;

- à la question des débouchés.

Les questions ainsi soulevées sont liées aux profils des étudiants admis à suivre l'enseignement et aux objectifs poursuivis par la formation. Il arrive que les étudiants bénéficient d'une formation initiale elle-même déjà interdisciplinaire qui les prépare plus ou moins à l'interdisciplinarité qu'ils retrouvent dans le master. Mais c'est l'exception. La règle générale en effet est l'ouverture la plus grande possible du recrutement, celui-ci se faisant en fonction du thème abordé et indépendamment de la discipline de formation initiale. De ce fait, si certains des étudiants ont déjà une formation dans certaines au moins des disciplines qui vont être enseignées dans le master, d'autres - et parfois la majorité - viennent d'horizons totalement différents. Dans tous les masters en cause ici, ces horizons vont des sciences physiques et biologiques aux sciences humaines et sociales. Certains masters incluent une perspective de formation continue en s'ouvrant à des personnes ayant déjà une expérience professionnelle; cela crée une forme de symbiose sans nul doute favorable à l'interdisciplinarité entre étudiants.

\footnotetext{
${ }^{7}$ Pour beaucoup dans le domaine de l'environnement, mais cela vaut aussi pour les autres masters répertoriés ici (en sciences cognitives, en archéologie, en écologie humaine, en didactique des sciences).

${ }^{8} \mathrm{Cf}$. ci-après les commentaires d'étudiants issus de ces formations.
} 
Dans certains cas, la multiplicité des disciplines prises en compte dans le master ne pose aucun problème pédagogique sérieux, dans la mesure où ces disciplines sont très proches les unes des autres et participent peu ou prou de la même culture scientifique ; on a alors affaire à une interdisciplinarité - et même le plus souvent, à une simple pluridisciplinarité - de proximité. Il en va de même lorsqu'il s'agit simplement d'approfondir une formation initiale déjà interdisciplinaire. Il n'y a guère plus de problèmes non plus quand il s'agit simplement d'ajouter des connaissances renvoyant à une nouvelle discipline à celles qui sont déjà acquises, par exemple des connaissances en économie ou en droit pour un étudiant venant des sciences de le Terre ou de l'écologie. On est là encore face à une pure et simple pluridisciplinarité et il s'agit alors tout au plus de pédagogie : il revient en effet à l'enseignant de susciter l'intérêt d'étudiants pour une matière nouvelle, ce qui suppose de sa part une prise en considération de qui ils sont et un souci de se greffer sur leurs centres d'intérêt. Cet effort d'adaptation s'impose surtout lorsque cette nouvelle discipline s'inscrit dans un univers de connaissance et une culture scientifique différents de ceux auxquels ils sont habitués (par exemple ceux des sciences humaines et sociales pour des étudiants de sciences de la Terre ou de biologie). Quand l'enseignant qui la dispense a lui-même une culture scientifique interdisciplinaire, il peut donner à son intervention une véritable dimension d'introduction à une réelle interdisciplinarité. Mais ceci apparaît très exceptionnel au vu des expériences examinées ici, dont un bon nombre s'inscrit en fait dans une démarche de pluridisciplinarité, et même de pluridisciplinarité proche.

Par contre, la situation se complique quand l'objectif est d'amener des étudiants de formations différentes à entrer dans une démarche interdisciplinaire commune et, qui plus est, dans une démarche impulsée par une discipline qui leur est étrangère. Il faut alors en passer par tout un travail de transformation des postures d'analyse et de mise en perspective des cultures scientifiques des uns et des autres. Le tronc commun a comme objectif d'en fournir les fondements. Mais d'autres méthodes sont aussi utilisées pour le réussir, tels les exercices de confrontations croisées, les expériences de terrain en groupe, la constitution de petits collectifs de travail fondés sur la convivialité et la durée, le partage de méthodes fédératrices : la modélisation, l'approche systémique, l'ingénierie vue comme une ouverture vers cette dernière, l'instrumentation (par exemple, les SIG), les mémoires co-dirigés. La pédagogie devient une maïeutique. Cela va jusqu'au recours à la philosophie, à l'épistémologie.

De difficulté à surmonter, l'hétérogénéité des profils des étudiants peut alors devenir une richesse, la diversité des cultures scientifiques fournissant la matière du dialogue. Le fait qu'elle aille de pair avec des rattachements institutionnels différents (universités, Grandes Écoles, laboratoires de recherche) renvoyant à des cultures scientifiques différentes et que les enseignements se dispersent dans l'ensemble des lieux qui incarnent cette diversité, ouvrent la curiosité en même temps que l'horizon et accroissent le désir de dialogue en même temps qu'il le facilite. Plus le master va dans cette direction, plus est sollicitée la capacité des étudiants à s'adapter et plus se pose la question de leur aptitude à le faire. Une exigence d'excellence peut finir par s'imposer.

Sans être poussée à un tel extrême, cette capacité d'adaptation des étudiants est un des traits généraux des formations interdisciplinaires. On peut même se demander si un des intérêts essentiels d'une formation interdisciplinaire n'est pas de la stimuler. Plus l'éventail des disciplines composant le tronc commun est large, plus les disciplines enseignées dans le master sont nouvelles, plus elles sont loin, dans le spectre scientifique, de celles de la formation initiale, plus l'effort d'adaptation à faire est important. Plus également la nécessité s'impose à l'étudiant de faire ses choix dans l'offre qui lui est proposée. D'où l'importance à la fois des options qui lui sont ouvertes et de la place qui est donnée dans le master à son travail personnel. 
De ce point de vue, les masters peuvent apparaître plus ou moins fermés ou, au contraire, ouverts. Certains offrent un protocole de formation aux exigences strictes et très ciblées, et visent un prototype relativement précis de profil. D'autres au contraire, sortes d' « auberges espagnoles », laissent une grande marge de détermination à chacun des étudiants : les options offrent un cadre de référence pour ce faire. Elles sont généralement corrélées avec le mémoire personnel. Leurs intitulés expriment des compromis prenant en compte à la fois les formations initiales majeures des étudiants et les objectifs de formation du master. Ils permettent donc à l'étudiant de se recaler par rapport à sa formation initiale. C'est là que se mesure l'effet réel de l'enseignement donné dans le master et notamment le bénéfice que l'étudiant a pu tirer de l'interdisciplinarité (ou même, plus modestement, de la pluridisciplinarité) dont cet enseignement lui a donné l'opportunité de profiter. Le risque évidemment est que le contenu du mémoire ne s'écarte guère de la discipline de départ, d'où l'intérêt, pour contrebalancer ce risque, de la double direction des mémoires.

C'est là qu'entre en ligne de compte la question de l'évaluation. En dépit de son importance, elle n'a été qu'effleurée au cours du colloque. Il faudrait entrer dans le détail des règles de notation des masters pour pouvoir apprécier de façon fine le poids respectif qu'ils donnent aux différents acquis. Il ressort néanmoins de ce qui a été dit que, considérés sous l'angle strict de l'interdisciplinarité, les résultats sont plutôt minces : les mémoires notamment restent généralement placés sous le signe des disciplines. Est-ce parce qu'ils sont souvent une première étape d'une future thèse ? On se heurte là aux cloisons de l'évaluation des thèses universitaires. Cette contrainte pèse moins sur les mémoires qui sont une fin en soi, notamment dans les masters professionnels. Qu'en est-il en particulier des mémoires qui ont été l'objet d'une co-direction?

Bien que rentrant dans l'ordre disciplinaire, les mémoires devant déboucher sur des thèses - et les thèses qui s'ensuivent -, peuvent se ressentir du passage par l'interdisciplinarité. Ceci se traduit par un «calage» de leurs problématiques différent de ce qu'il serait dans le cas d'une démarche proprement disciplinaire, car elles sont davantage situées dans une démarche d'ensemble autour de la question abordée. Sur le plan proprement universitaire, un des intérêts - a minima - des confrontations interdisciplinaires que mettraient en évidence les masters interdisciplinaires serait donc au moins le recadrage auxquels elles obligent les disciplines en leur faisant prendre conscience de leurs limites. Ce n'est pas anodin. Mais on est loin de l'ambition de créer une génération de jeunes chercheurs à profils interdisciplinaires larges (associant, notamment, selon des modalités diverses, sciences de la nature et sciences humaines et sociales). Cette ambition tournerait-elle court face aux résistances des structures universitaires ? Le fait est que le jeu apparait nettement plus ouvert du côté des Grandes Écoles, mais aussi des masters universitaires professionnels, ou même mixtes. Y a-t-il là un double vivier possible pour la recherche?

\section{Caractéristiques générales de l'interdisciplinarité qui ressortent de ces expériences de formation}

$\mathrm{Au}$ simple vu du descriptif de leur programme d'enseignements et des méthodes pédagogiques utilisées, les masters peuvent être classés sur une échelle allant de la plus sommaire pluridisciplinarité à une interdisciplinarité approfondie, en passant par des états intermédiaires. Une analyse fine des contenus des enseignements et des pratiques pédagogiques serait nécessaire pour pouvoir en dire plus sur ce point, évidemment essentiel. Ni les présentations, ni les débats n'ont permis d'avancer dans cette analyse. Tout au plus peut-on dire que, si l'on définit comme véritablement interdisciplinaires les masters dans lesquels il existe des exercices de réflexivité croisée entre disciplines, rares sont ceux qui 
répondent à ce critère. Ceci n'est pas une critique, car une pluridisciplinarité bien conçue représente déjà une avancée ${ }^{9}$; c'est un constat qui peut servir de base à la réflexion.

En fait, le trait qui frappe le plus à l'issue de ce tour d'horizon, c'est l'extrême diversité des formules pédagogiques expérimentées (sauf en ce qui concerne le tronc commun, qui se retrouve dans la plupart des masters). On est tout à l'opposé des enseignements classiques, structurés autour des fondamentaux généraux d'une discipline, que l'on retrouve à l'identique, à quelques variantes près, dans toutes les universités. La physionomie de chacun des masters s'explique à l'évidence par le contexte universitaire dans lequel il s'inscrit, par l'histoire de ce contexte, par le contexte régional dans lequel l'université elle-même se situe, par la discipline de rattachement - mais aussi par la personnalité - des enseignants qui en prennent l'initiative et de ceux qui lui donnent corps. Chacun de ces masters a ses objectifs, sa thématique, sa " philosophie » et son histoire propres. Il n'y a pas de modèle ; on est dans le domaine du bricolage. Avec tout ce que ce mot implique de compromis, par rapport à l'objectif poursuivi, imposés par les contraintes locales de tous ordres. Mais aussi avec l'exigence de créativité que cela suppose pour réussir à mettre sur pied une formule crédible en tirant le meilleur parti possible des atouts dont on dispose. Le désir d'innover est premier, mais le pragmatisme est la règle ; il est la condition de l'efficacité.

Ce pragmatisme ne concerne pas seulement les modalités de l'enseignement, il s'impose aussi pour son contenu. Tout d'abord parce que celui-ci est conçu en fonction de l'objectif poursuivi (en tenant compte des compétences et des moyens disponibles, comme on vient de le voir). Mais aussi parce que le domaine thématique de référence du plus grand nombre des masters examinés (des questions en rapport avec l'environnement, la santé, l'aménagement, et tout ceci dans une perspective de développement durable) est vaste et sollicite de nombreuses disciplines. Aucun master ne peut prétendre le couvrir à soi seul. Et aucun ne le prétend. Le découpage alors opéré procède largement de la discipline qui est à l'initiative du master et donc, du département universitaire auquel il est rattaché. On a en somme affaire à ce que l'on pourrait appeler une « interdisciplinarité centrée », c'est-à-dire centrée sur une discipline majeure qui sert de point d'appui pour des élargissements disciplinaires considérés à la fois comme nécessaires et comme suffisants compte tenu de l'objectif visé. L'intérêt de l'archéologie est d'offrir un exemple où, au contraire, un espace interdisciplinaire constitutif d'une discipline a fini par se stabiliser. Mais il montre que, même dans ce cas, des variantes demeurent d'un master à l'autre. L'exemple des sciences cognitives - et cela vaut, à un moindre titre toutefois, pour l'écologie humaine - illustre un autre cas de figure caractérisé par le foisonnement, la mobilité, la vitalité, l'instabilité créatrice d'une interdisciplinarité en constant mouvement de renouvellement. Dans ces deux cas, la référence à une appellation unitaire fait plus office d'un appel à un travail en continu de croisements de regards disciplinaires autour d'objets et, surtout, de questionnements communs, qu'il n'exprime le souci d'un démarquage disciplinaire. Ce foisonnement, cette mobilité, cette vitalité, cette fluidité, on les retrouve dans les masters portant sur l'environnement et le développement durable si on les considère dans leur ensemble. Mais en fait, ils ne forment pas un ensemble, chacun d'eux n'existant que par et pour lui-même. D'où l'absence de dynamique créative collective, ce qui bride la dynamique créative au niveau de chaque master en particulier. Le pragmatisme est gage de souplesse. Il permet de se couler dans des niches de formation qui se situent sur les marges, dans les interstices, des grands blocs institués, de les occuper et de leur donner corps. Mais on n'est pas là à l'échelle du continent, c'est plutôt l'image de l'archipel qui vient à l'esprit. Avec les limites que cela signifie du point de vue de la création collective.

\footnotetext{
${ }^{9}$ « Nous apprenons aux étudiants à faire ce que nous ne savons pas faire » a dit une intervenante.
} 
Ce caractère fragmentaire des formations universitaires interdisciplinaires ne doit pas faire oublier qu'elles procèdent toutes d'une même nécessité: mettre la formation à la recherche et la formation par la recherche en phase avec l'évolution des questions que suscite l'avancée des connaissances et avec la façon dont les sociétés s'en emparent pour penser les problèmes que leur propre développement soulève. De ce point de vue, celles qui ont servi de matière à la réflexion de ces journées sont exemplaires dans la mesure où elles reflètent un mouvement en profondeur au sein tant des sciences que des sociétés. Ce mouvement est indissociable de l'interdisciplinarité. C'est ce qu'elles traduisent clairement en se plaçant sous le signe de cette dernière. En utilisant les opportunités qui se présentent de commencer à l'impulser sur un point précis, ce sont autant de « prises » qu'elles lui assurent dans le champ de production et de transmission des connaissances, fusse en cantonnant leur ambition dans les limites d'une pluridisciplinarité raisonnable.

Faut-il dépasser ce stade pour aller plus avant dans la voie de l'interdisciplinarité. Les masters les plus engagés dans cette direction incitent à répondre positivement dans la mesure où, précisément, ils éprouvent le besoin de s'y engager pour atteindre leur propre objectif ; dans la mesure aussi où, ce faisant, ils font la démonstration de la nécessité d'une clarification des conditions du travail interdisciplinaire passant par un réexamen des fondements des démarches des disciplines. L'argument le plus général pour aller dans ce sens est de dire que, si l'interdisciplinarité est une nécessité, alors elle doit être construite rigoureusement en tant que méthode scientifique puisqu'elle en est une et qu'elle prétend, en tant que telle, avoir ses propres canons. Lesquels constituent par définition les critères de son évaluation, évaluation sans laquelle il n'y a pas science. Répondre à cette exigence implique d'utiliser la formation interdisciplinaire ou même simplement pluridisciplinaire (en termes de contenus), pour accéder à la formation à l'interdisciplinarité (en termes de méthode).

Comme le montre ce compte-rendu, les débats ont simplement permis de commencer à dresser un état des lieux, sans doute fort incomplet. Ils n'ont pas permis d'aller jusqu'à la formulation de ces questions de fond. Mais ce sont bien elles qui sont au cœur de l'échange d'expériences qui a nourri le colloque. D'où l'intérêt des présentations sur lesquelles se sont fondés ces échanges et qui elles offrent un matériau pour aller plus loin dans la réflexion. 


\section{Interventions choisies}

\section{Disciplines et enseignements pluridisciplinaires}

Henri BUC, Professeur honoraire à l'Institut Pasteur

Comme l'ont bien montré les Journées organisées par Natures Sciences et Sociétés en février 2007, il est opportun d'entreprendre maintenant une réflexion sur les spécificités des formations pluridisciplinaires. Il paraît cependant difficile de le faire sans avoir d'abord pris la mesure de ce que veulent et de ce que peuvent déjà assurer les formations dispensées dans un cadre strictement disciplinaire. Les réponses données à des questions très naïves dépendent de ce recadrage. Citons par exemple deux interrogations : l'enseignement prodigué dans le cadre des disciplines doit-il nécessairement précéder les enseignements de type pluri- ou multidisciplinaire? Les pédagogues qui ont accumulé des expériences dans ces nouvelles formations sont-ils à même de proposer des inflexions dans les méthodes et dans le contenu des programmes propres à chaque discipline?

\section{Quelques remarques sur la notion de discipline}

Remarquons tout d'abord que le cadre disciplinaire est lui-même assez flou. On s'accorde en effet difficilement sur la définition du concept de discipline et sur la cohérence de ce concept, lorsque l'on passe du domaine de la recherche à celui de l'enseignement. En reprenant une analyse de Jean Gayon ${ }^{10}$, je voudrais souligner que deux lignes de force coexistent dans la définition de cette notion : c'est d'abord depuis l'Antiquité un ensemble de savoirs, transmis de génération en génération par des experts reconnus. Mais c'est aussi, en une acception plus moderne, un domaine de recherche, ou plus pragmatiquement, un régime de technologies coordonnées, orientées vers la résolution de problèmes.

On voit bien que, selon la première ligne de force, l'enseignement des savoirs est littéralement constitutif de la discipline. Dans un monde où la connaissance évolue lentement, le passage de cette maîtrise d'une génération à la suivante s'opère selon des procédures éprouvées. La constitution et la survie du domaine concerné dépendent de l'efficacité de ce processus, même si, bien entendu, toute modification significative du contenu de la connaissance dans le domaine considéré doit se retrouver dans l'enseignement correspondant. La discipline constitue bien un champ, au sens de Bourdieu ${ }^{11}$. En découle un grand nombre de caractéristiques institutionnelles, sociétés, chaires, journaux spécialisés, sections dans les Établissements publics à caractère scientifique et technique (EPST) $)^{12}$.

Dans la définition hybride de la discipline donnée plus haut, le second terme vient atténuer le caractère statique et hiérarchisé du premier : tout domaine de la connaissance est

10 J. Gayon. Par exemple, History and Epistemology of Molecular Biology and beyond. Problems and Perspectives, Preprint 310, 2005, 249-252, Max Planck Institute for the History of Science.

${ }^{11}$ P. Bourdieu, Les usages sociaux de la science, Conférence-débat, 1997, Éditions Inra, rue de l'Université, 75338 Paris Cedex 07.

${ }^{12}$ La prévalence d'un enseignement par disciplines confère en retour un prestige social exagéré aux cadres disciplinaires retenus. L'évaluation des élèves est aisée selon cette division. Marc est bon en maths et mauvais en latin. Il est alors tentant de considérer que l'on pourrait déterminer les potentialités créatrices de Marc à partir de ces évaluations discrètes qui joueraient, en quelque sorte en ce domaine, le rôle de couleurs primaires dans la palette d'un tableau. 
en perpétuelle évolution et sa construction s'effectue aujourd'hui majoritairement entre pairs et non via le couple maître-disciple. Mais, à y regarder de plus près, un tel régime n'est plus de nos jours l'apanage des seuls processus disciplinaires. Certes, il s'est constitué d'abord au cœur de disciplines précises, mais il en déborde aujourd'hui nettement le cadre. Il s'établit naturellement chaque fois que s'accélère la vitesse d'acquisition et de transmission de nos connaissances.

En fait, le savoir disciplinaire est confronté à deux types d'évolution, aux rythmes souvent très différents: d'une part, l'évolution relativement lente, propre au champ disciplinaire ; d'autre part, l'évolution beaucoup plus chaotique des relations entre la création et la dispensation d'un savoir disciplinaire donné. C'est dans cette différence de tempo que s'insère naturellement le recours à l'interdisciplinarité. Elle est évidemment de mise en recherche. Car, s'il est un fait caractéristique de la modernité, c'est bien que la croissance protéiforme des savoirs se moque des frontières des disciplines. Elle ne cesse d'en créer de nouvelles, d'en modifier le contenu, d'en bousculer les limites. Mais, d'un autre côté, nous ne pouvons pas faire comme si ce divorce se résolvait sans questionnement dans le cadre de l'enseignement. Nous ne pouvons pas nous résoudre à une forme d'enseignement qui ne serait que la dispensation de savoirs émiettés, à travers des formations de toutes sortes. Car, dans une discipline donnée, l'enseignant doit aussi réintégrer tout ou partie de ces avancées de la recherche dans le savoir qu'il dispense comme dans la pédagogie qu'il pratique.

Cet autre mode d'interaction entre recherche et enseignement, ce remodelage des disciplines existe depuis toujours. Si nous remontons très haut dans l'histoire des Sciences, nous constatons que les disciplines se sont constituées selon des a priori qui ne sont plus du tout les nôtres. La chimie et la physique surgissent à l'origine de questionnements très différents, pour faire simple la transmutation de la matière dans un cas, le mouvement des astres dans l'autre. Des siècles s'écouleront avant que leurs problématiques ne convergent. De son côté, la biologie a mis des siècles pour en finir avec le vitalisme, un concept crucial pour son émergence en tant que discipline. Voici que maintenant, comme la chimie il y a quelques décennies, elle commence à perdre son statut de science naturelle. On voit en effet émerger aujourd'hui des micro-organismes entièrement synthétisés au laboratoire et qui pourront fonctionner selon des schémas métaboliques inédits!

La visée et le contenu de chaque discipline ne cessent donc de se modifier. Chacune conserve de fait une trace en quelque sorte solidifiée de son origine et de l'évolution des connaissances qu'elle entend regrouper. Fait tout aussi remarquable, les grandes disciplines survivent à ce remue-ménage. Elles continuent à correspondre à des modes de saisie bien spécifiques du réel : on parlera du regard caractéristique porté par un mathématicien ou par un physicien sur un problème complexe. Un processus continu de réordonnancement des connaissances, de leur réinsertion dans un corpus de disciplines préexistantes et séparées est donc constamment à l'œuvre. Ceci commence par le renouvellement des paradigmes dans la recherche, se continue par l'élaboration de nouvelles grandes synthèses universitaires et se termine par une nouvelle façon d'enseigner au cœur des disciplines : ce que nous devons enseigner aujourd'hui, ce n'est pas avant tout ce qui nous a nourris, mais ce qui constituera, pour la génération qui vient, le meilleur outil conceptuel.

\section{Implications pédagogiques}

Tout pédagogue doit donc faire face à un sérieux paradoxe : à mesure que la connaissance progresse, les frontières entre disciplines s'estompent; et pourtant toute l'organisation de nos savoirs, depuis que nous avons quitté le jardin d'enfants, continue à se faire dans le cadre de quelques disciplines majeures. Une pratique de la science, une façon d'en communiquer les résultats, une façon de l'enseigner ont été historiquement éprouvées dans ce cadre-là. Elles ont laissé dans notre pédagogie une trace indélébile. Le pédagogue sépare, isole, et en un sens 
reprend la démarche historique de conquête des savoirs. Il le fait généralement à bon droit : dans une discipline donnée, les questions posées sont claires; elles apparaissent cruciales à tout un chacun ; les méthodes d'enseignement y sont éprouvées.

Dans le monde changeant de la modernité, la tâche du pédagogue est alors triple : assurer un enseignement rigoureux de sa discipline; s'assurer que l'élève a non seulement assimilé ce nouveau socle de connaissances mais qu'il est aussi capable de le rendre opérationnel ; éprouver enfin avec lui comment ces savoirs partiels ne trouvent leur pleine efficacité qu'en se conjuguant avec d'autres savoirs.

Il nous faudra donc réfléchir dans le futur à la façon dont ces trois objectifs pourraient se répartir au fil du temps entre enseignement disciplinaire, pluridisciplinaire et multidisciplinaire.

Dans un régime classique, l'acquisition du « socle commun de connaissances » se fait dans le cadre disciplinaire. Ce n'est qu'ensuite que l'élève apprend à conjuguer ses divers acquis. Notons d'abord qu'en pratique les deux derniers types d'enseignement sont souvent appelés à la rescousse simplement parce que l'enseignement prodigué dans le cadre disciplinaire s'est concentré sur son premier objectif au détriment des deux autres. La constitution d'un socle de connaissances est en effet le processus le plus simple à évaluer des trois, mais, répétons-le, séparé des deux autres, il fige l'esprit de l'élève dans un savoir de type médiéval, il brise le questionnement créatif, il compromet même in fine ce processus de ré ordonnancement des savoirs que toute université doit s'honorer de prendre en charge. Notons ensuite qu'il va être nécessaire d'envisager la complétion graduelle de ces objectifs dans un temps long, celui de la scolarisation. Ceci implique probablement la création de nombreuses boucles de rétroaction entre les différents types d'enseignements envisagés, et non l'abandon progressif de l'enseignement des disciplines majeures au profil d'un enseignement pluridisciplinaire au cours de la scolarité.

Lors des journées de février 2007, de nombreuses expériences novatrices ont été présentées et discutées. Marcel Jollivet les a résumées dans un document récent. Reprenant l'exemple traité par François Taddei ${ }^{13}$, celui de la transformation de la biologie, je voudrais pour ma part examiner les défis spécifiques qui se profilent lorsqu'une discipline subit de rapides et profonds remaniements. En effet, lorsque nous parlons de transmission des savoirs, nous raisonnons sur un temps long, celui qui est requis pour qu'un ensemble de disciples remplace la génération des maîtres qui les ont formés, soit une quinzaine d'années environ. Que s'est-il passé lors des transformations récentes de la biologie qui ont vu d'abord le triomphe de la biologie moléculaire dans les années 1960, et plus récemment la constitution d'un nouveau type de savoirs, la biologie des systèmes? Durant un intervalle de moins de quinze ans, et par deux fois, le contenu des savoirs a littéralement explosé. Dans les deux cas, la synergie des compétences entre physiciens, chimistes et biologistes opère à plein sur le terrain, mais la remise en ordre des concepts de base du domaine qui devrait en résulter tarde à s'effectuer. Elle n'est pour un temps maîtrisée, ni par les acteurs, ni a fortiori par les universitaires. Dans un tel contexte, il n'existe plus de procédure éprouvée pour la transmission de ces nouveaux acquis. De fait, nous avons vu la Biologie moléculaire se constituer lentement en un champ disciplinaire spécifique. En France, cette étape demanda quinze ans environ, soit le temps requis pour former directement, à partir des chercheurs concernés, une première génération de disciples. Puis, ce nouveau champ a été à son tour victime de son succès. Avant la fin $\mathrm{du} \mathrm{XX}^{\mathrm{e}}$ siècle en effet, il éclate à son tour lorsque se constitue, sous une forme tout aussi chaotique, un nouveau savoir sur le vivant impliquant

\footnotetext{
${ }^{13}$ F. Taddei, Approche interdisciplinaire du vivant (dans ce volume).
} 
d'autres mathématiciens, d'autres physiciens et d'autres chimistes ${ }^{14}$. Lorsque naissent et meurent à ce rythme les sous-disciplines, la transmission de savoirs pertinents pour le champ concerné implique des aménagements radicalement différents de ceux qui peuvent être préconisés dans le cas d'une évolution plus tranquille. Mais la crise temporaire sera d'autant plus difficile à dénouer que les savoirs dispensés antérieurement l'auront été sous une forme trop scolastique et trop confinée.

Que disent au fond les chercheurs, dans leur grande majorité, lorsqu'on sollicite leur avis dans le champ de la pédagogie ? D'abord, que nous savons encore bien peu de choses (même si le contenu de ces savoirs est déjà bien vaste pour être commodément maintenu dans le cadre des programmes existants), que ce savoir est souvent approximatif et qu'il est toujours soumis au crible de la réfutation, cher à Popper. Plus nous nous éloignons des sciences dures, et plus le mode de description et de raisonnement que nous utilisons doit luimême être soumis à réfutation critique ${ }^{15}$. C'est lorsque nous sommes humbles et lucides que notre créativité s'aiguise, et son efficacité sera d'autant plus forte que nous saurons marier sur des interrogations précises des savoirs divers. Enfin, cette créativité participe à son tour, à tous les échelons de la transmission des savoirs, au renouvellement des disciplines. Je pense que c'est en suivant cette démarche-là que nous pourrons significativement accélérer les avancées du savoir.

\section{Une expérience particulière d'interdisciplinarité}

Je pense personnellement que ces changements très profonds dans l'acquisition des connaissances impliquent de fait l'abandon d'une pédagogie plus classique, centrée sur la dispensation du savoir par le maître. Il me semble même qu'il faudra renoncer à faire succéder dans le temps des enseignements dispensés par disciplines et des expériences à forte connotation pluri- ou interdisciplinaires. Ceci ne se fera vraisemblablement pas sans mal. J'ai participé, de 1945 à 1950, à une expérience hardie de pédagogie novatrice. À la Libération ressurgirent en effet de nombreux projets, abandonnés dans les cartons de la III $^{\mathrm{e}}$ République. Ainsi, un projet pédagogique proposé par le ministère Jean Zay en 1939 est-il relancé par les travaux d'une commission de réforme pédagogique, nommée à la fin de 1944 et présidée par le physicien Paul Langevin et le médecin Henri Wallon. Dès 1945,188 classes de sixième dites nouvelles, soumises à des principes pédagogiques très précis, sont créées dans différents lycées de France: à l'initiative de son Proviseur, le lycée de Montluçon se lance dans l'expérience. Rappelons d'abord la visée de cette pédagogie. Un ancien professeur de sciences de ce lycée, Monsieur Galéazzini, en a bien résumé l'esprit :

Les cours ex cathedra, dont la qualité n'était nullement mise en cause, avaient pour conséquence de rebuter certains élèves. Le but évident de cette nouvelle pédagogie était d'éviter le désintérêt, d'éveiller la curiosité, de donner le goût de la recherche et de l'étude, d'apprendre à réfléchir, de passer de l'à peu près à la rigueur... Certains professeurs avaient été tentés par des méthodes leur laissant beaucoup de liberté dans leurs initiatives. Il ne fallait plus asséner des faits à un auditoire plus ou moins motivé, mais il fallait laisser les élèves découvrir, participer, conclure, avec l'aide du maître.

\footnotetext{
${ }^{14}$ En termes d'organisation de la Science, il ne s'agit pas d'une mince affaire. Elle conditionne par exemple le dessin de nouveaux campus, où l'optimalisation de ce type d'interactivité pour une nouvelle génération de scientifiques conduit à la réalisation de complexes architecturaux totalement originaux.

${ }^{15}$ Quelques exemples : la constitution des neurosciences requiert la synergie et la hiérarchisation de disciplines connexes dans une combinatoire encore mal maîtrisée aujourd'hui (voir l'exposé de D. Andler au colloque NSS précité à paraître dans la revue NSS). Dans un appendice à son ouvrage, Comment on écrit l'Histoire, Paul Veyne, rendant hommage à Foucault, montre que: "l'intérêt de l'histoire n'est pas dans l'élaboration d'invariants, mais dans l'utilisation de ceux-ci pour dissoudre des rationalismes (trompeurs) sans cesse renaissants ».
} 
Dans un souci de réalisme, les professeurs en charge de cette expérience locale avaient cependant considérablement atténué certaines consignes radicales prodiguées par les promoteurs de l'expérience au niveau national. Bien des années plus tard, quelques-uns d'entre nous ont voulu savoir, au travers d'une enquête envoyée aux anciens élèves qui avaient participé à cette expérience locale, ce qu'ils en avaient retenu et comment ils la jugeaient ${ }^{16}$. Un travail voisin a été effectué par les élèves filles du lycée Honoré de Balzac de Tours, qui ont suivi, de 1948 à 1953, un cursus analogue ${ }^{17}$.

Les réponses à notre questionnaire, aussi bien que les témoignages récoltés à Tours, montrent le réel succès rencontré par ces initiatives auprès des participants. Il ne s'agit pas seulement du souvenir heureux qu'elles ont laissé à ces anciens élèves, mais aussi des atouts spécifiques qu'elles leur ont procurés pour la réussite de leurs études ultérieures ou de leurs carrières professionnelles. Ont été ainsi plébiscités l'introduction du travail manuel dans la scolarité, l'étude du milieu naturel et humain, le travail sur des thématiques impliquant plusieurs professeurs et les interactions fortes engendrées par le travail en équipes. Donnonsen quelques illustrations : le passage du style roman au style gothique, étudié en cinquième en histoire, fut l'occasion de commencer à comprendre, avec le professeur de sciences, comment les vides avaient pu gagner sur les pleins dans ces édifices, par une étude de la composition des forces s'exerçant dans ces architectures. Les dissertations étaient travaillées jusqu'au point où elles recevaient un «bon à tirer » du professeur de français, et cette mise au propre engendrait un travail de copiste et d'illustrateur mené avec la coopération du professeur de dessin. Voyages organisés ou activités théâtrales participaient du même esprit. Par le travail en équipes, nous étions conduits à nous intéresser non seulement à nos propres progrès, mais aussi à la progression de notre équipe et de toute la classe. Un sens concret de la responsabilité s'est ainsi développé. La plus grande liberté d'action laissée aux enfants a été en général évaluée positivement, quelque soixante ans plus tard : «Plus que liberté, il faudrait dire adhésion à un ordre consenti. Ceci nécessitait en fait beaucoup de vigilance, note un ancien élève. Un autre ajoute: En fait, nous intériorisions des contraintes nécessaires et reconnues, car nous avions à atteindre des buts bien définis, et nous étions soumis à une permanente exigence de qualité. » Seule réticence manifestée à ce sujet : un travail effectué en équipe masque parfois des lacunes spécifiques de l'élève. Il aurait exigé en fait un contrôle continu des connaissances ; un tutoring individuel aurait été requis pour atténuer les inégalités liées à la différence de statut social des élèves au départ de l'expérience. De tous ces témoignages et échanges, se dégage donc une appréciation positive de l'expérience pluridisciplinaire à laquelle nous fûmes soumis à Tours comme à Montluçon. Elle nous a ouvert les yeux sur le monde. Elle nous a responsabilisés. À travers ces projets, nous avons saisi la complémentarité des disciplines scolaires, ce qui constitua pour beaucoup une excellente préparation à la vie professionnelle future.

Comment se fait-il donc, qu'au plan national comme au plan local, cette expérience d'enseignement pluridisciplinaire ait été rapidement abandonnée ? Pourquoi a-t-elle été quasiment oubliée, et jamais reprise dans un esprit analogue ? Pourquoi sommes-nous aujourd'hui si loin de l'optimisme qui prévalait au lendemain de la Libération?

Nous nous sommes à nouveau interrogés sur ces points. Au plan national, un rapport assez réservé fut rédigé par Maurice Lacroix dès 1949. Le cursus des classes nouvelles allait de la sixième à la seconde. En 1949, aucune classe n'avait encore terminé ce cursus, si bien que cette évaluation était un peu prématurée. Le rapport reconnaissait de nombreux points

\footnotetext{
${ }^{16}$ H. Buc, Les classes Nouvelles au Lycée de Montluçon. (2005) Bulletin des Anciens Élèves de ce Lycée. Texte disponible sur demande ; C. Chauvet, L'expérience des classes nouvelles à la Libération, à travers l'exemple de Montluçon (1945-1952). Maîtrise d'Histoire contemporaine. Université Blaise Pascal, Clermont 2 (2004).

${ }^{17}$ Classes Nouvelles et Gai Savoir au féminin. Ouvrage collectif, L'Harmattan (2004).
} 
positifs à l'expérience, mais soulignait les dangers de l'esprit de système, la trop grande part de travaux effectués en équipe, les dangers de l'utilisation polyvalente des professeurs, Mais, dans les deux expériences citées, les excès en la matière avaient été rapidement corrigés. Le test crucial allait en fait être mené sur le terrain, lors de l'intégration à partir de 1950 de ces élèves dans le cursus classique. Dans les deux cas analysés, cette intégration se fit sans mal, les élèves concernés prenant souvent la tête de leur nouvelle classe. Mais les réserves allaient s'accumuler, pour des raisons plus triviales. L'expérience était en fait coûteuse pour l'époque, car elle requérait des classes à effectifs restreints; elle exigeait aussi des professeurs une constante motivation. Elle rencontra surtout l'hostilité déterminée de la société des agrégés. On était en effet très loin des options conservatrices de cette institution. En fait, toute pédagogie qui s'intéresse avant tout au développement de la créativité de l'enfant ou de l'étudiant fait perdre un certain nombre de repères, considérés comme indispensables dans un enseignement plus conservateur. "Laisser les élèves découvrir, participer, conclure, avec l'aide du maître », comme il a été défini plus haut, appelle en fait la constitution d'un système d'interactions beaucoup plus exigeant entre l'équipe professorale et les élèves, et non pas plus laxiste, comme l'ont propagé dans une caricature de cette expérience les tenants du système antérieur : professeurs et élèves ont plus de travail. Les évaluations deviennent plus délicates, car l'innovation est le fait d'une collectivité et non plus d'un individu; elle conduit rarement aux résultats que le maître attendait. Celui-ci perd donc certains repères qui lui étaient fort utiles dans le cadre de la pédagogie classique. Est-ce cher payé, au regard des progrès que j'ai décrits un peu plus haut? Attendons-nous pourtant, dans le contexte difficile qui est fait aujourd'hui à toute pédagogie active, à rencontrer dans le futur les mêmes types de réactions négatives. Préparons-nous à y répondre en faisant évaluer de façon objective toutes ces expériences et en en consignant scrupuleusement la teneur.

La transmission intelligente de savoirs, dans notre monde moderne, appelle un renouvellement constant des corpus transmis. On ne saurait opposer dans un mauvais procès, la constitution d'un socle commun de connaissances à la nécessaire recomposition des savoirs, d'une génération à la suivante. À nous d'inventer les modalités les plus efficaces pour assurer cette complémentarité !

\section{Le master Communication scientifique et technique : contenus, outils, pratiques}

Jean-Louis MARTINAND, Professeur de didactique des sciences et techniques, École normale supérieure

de Cachan

Je suis intéressé par le thème des journées et de la table ronde pour de multiples raisons : passé de la physique aux sciences de l'éducation, j'ai eu l'occasion d'expertiser divers masters ou laboratoires dont une des caractéristiques majeures est la pluridisciplinarité. Comme directeur d'un laboratoire de didactique des sciences et techniques, puis comme responsable du Centre d'Alembert (Centre interdisciplinaire d'étude de l'évolution des idées, des sciences et des techniques, université Paris-Sud), j'ai consacré une part de mon temps à la réflexion que nécessitent de telles organisations.

Cependant, je souhaite ici focaliser l'attention sur quelques aspects du master «Communication scientifique et technique : contenus, outils, pratiques », que j'ai monté à l'ENS de Cachan. Recrutant en M 2 après un M 1 des domaines Sciences, Technologies, Santé, il comporte à la fois un parcours professionnel qui vise à valoriser en France et à l'étranger des étudiants qui ont fait une bonne préparation d'agrégation en leur donnant des 
capacités de construction ou d'instrumentation de formations ou de dispositifs de diffusion, et un parcours recherche Didactique des sciences et techniques. Ce second parcours prend en réalité la suite d'un Diplôme d'études approfondies (DEA) dont la première apparition date de 1975 ; il comporte quatre options : didactique des sciences expérimentales et des disciplines techniques; médiatique des sciences et techniques avec le Palais de la Découverte; technologie de l'information pour l'enseignement et la diffusion; histoire de l'enseignement et de la diffusion des sciences et des techniques. Dans cette option recherche, qui est la seule dont je parlerai, la majorité des étudiants est composée, à côté de quelques normaliens qui ne veulent pas faire de recherche "classique », d'enseignants et de formateurs en activité, de France et de pays de la francophonie.

À première vue, un tel master doit à l'évidence être interdisciplinaire, et même de multiples manières :

- par son champ d'intérêt, les sciences, les techniques, leurs enseignements et leur diffusion ; - par les disciplines d'origine des étudiants, scientifiques ou technologiques et parfois médicales ;

- par les compétences visées pour les étudiants, recherche, conception de contenus et de dispositifs en éducation générale, formations spécialisées, ou actions culturelles ;

- par les contenus de formation enfin, qui mobilisent les méthodes, les instruments et certains concepts des sciences humaines et sociales ou de l'ingénierie culturelle, tout en gardant la maîtrise des problématiques.

Le master doit encore être qualifié d'interdisciplinaire par l'ambition de traiter comme des problèmes internes les rapports entre les champs scientifiques, technologiques, professionnels et culturels, et par l'amalgame dans une équipe enseignante de chercheurs d'origines diverses (informatique, physique, chimie, biologie, psychologie, sociologie, histoire des sciences), même si une partie importante est maintenant en sciences de l'éducation.

Cependant, il faut noter les modalités et les raisons diverses pour un tel master d'être interdisciplinaire. Et surtout, il faut poser la question : au-delà des évidences, l'intention générale, la problématique fondatrice, est-elle vraiment interdisciplinaire, ou au contraire foncièrement disciplinaire? Autrement dit, s'agit-il d'un montage qui intègre diverses disciplines pour un projet inaccessible à chacune isolément, ou ne s'agit-il pas d'un projet de fondation d'une autre discipline, fondation qui ne peut évidemment advenir de rien ni se poursuivre sans emprunts, mais qui vise à promouvoir et conforter l'autonomie de problématiques spécifiques. Je peux certifier que tel était bien le projet des «didacticiens » vers 1975 : et ils ont utilisé, à cet effet, ces outils majeurs de configuration et de renouvellement d'une discipline académique qu'étaient à l'époque les DEA.

Avec le recul, on peut dire que les pratiques des équipes de recherche montrent trois grandes conceptions ou trois grands types de recherche didactique :

- la production de faits validés; c'est le type majoritaire, selon une conception «scientifique» au sens français classique. Cette conception est souvent accompagnée d'illusions sur la possibilité d' " applications ", d'assez nombreuses impasses sur les pièges habituels des recherches en sciences humaines et sociales, et de croyances assez fortes dans la valeur des résultats produits. On peut penser qu'il n'y aurait pas vraiment besoin de recherches didactiques du premier type si les sciences humaines et sociales voulaient bien s'intéresser aux questions d'éducation et formation spécifiques des contenus, au-delà de premiers apprentissages ;

- la mise au point de propositions évaluées de contenus et dispositifs éducatifs ; c'est un type minoritaire, mais renouvelé en permanence, pour répondre à des besoins nouveaux. On pourrait penser qu'il suffit pour cela de la créativité individuelle des enseignants. En réalité, 
l'invention, l'exploration et la mise à l'épreuve collectives et armées des possibles, s'avèrent nécessaires dans les contextes divers, changeants et contradictoires de notre époque ;

- le renouvellement du questionnement sur les contenus et les dispositifs éducatifs, leurs conséquences et leurs conditions, leurs implications sur les processus et leurs acteurs; c'est ici sans doute qu'est la contribution majeure, et donc l'enjeu décisif de la recherche didactique, celui qui ouvre la voie aux deux types de contributions précédentes; or la condition de possibilité de questionnements renouvelés, c'est la mise au point de concepts nouveaux. C'est en ce sens que la maîtrise des problématiques est une affaire décisive, pour la didactique comme pour toute discipline.

Or, l'expérience des recherches en éducation qui veulent prendre en compte la spécificité des contenus, ou, dit plus fortement, qui veulent assumer une responsabilité concernant les contenus d'enseignement, suggère qu'il est très facile de perdre la maitrise des problématiques ou d'abdiquer devant les responsabilités de l'invention. Ainsi, les chimistes, alors même qu'ils étaient beaucoup plus nombreux que les physiciens ou les biologistes à chercher à innover dans leur enseignement à tous niveaux et pas seulement en France, ont longtemps cru qu'il suffisait de monter des coopérations entre chimistes et spécialistes de l'éducation, par exemple sur l'évaluation : la didactique de la chimie a pris ainsi du retard, en hésitant à poser des questions spécifiques de la chimie elle-même.

Une autre manière très instructive de perdre la main est de penser avec ce produit de l' « entendement professoral » qu'est le «triangle didactique » (qualifié aussi par d'autres de « triangle pédagogique »), formé de l'enseignant, de l'apprenant, et du « savoir ». Beaucoup n'ont pas hésité à en inférer que la connaissance de l'apprenant relevait de la psychologie, celle de l'enseignant de la pédagogie, et celle du savoir de l'épistémologie : n'est-ce pas, par sous-traitance et naturalisation des « objets » de recherche, dire sans s'en apercevoir que la didactique est totalement inutile ou, en tout cas, qu'elle n'a pas d'initiative problématique propre?

J'entends l'objection: encore une discipline de plus! Pas si simple. D'abord, il ne semble pas que les didactiques ainsi caractérisées, par l'exercice d'une « responsabilité sur les contenus », puissent être considérées comme des disciplines "autoreproductrices », sous peine de devenir autistes et absolument stériles. Ensuite, il semble que les exigences comparatistes et transdisciplinaires soient aujourd'hui de plus en plus ressenties par les chercheurs et les innovateurs. De ce point de vue, les didactiques de disciplines sont peut-être une forme de transition.

Reste ce challenge majeur de penser toutes les formes possibles des actions éducatives et formatrices, leurs conditions, leurs processus et leurs évolutions. Ces actions ne sont jamais sans contenus, même lorsque qu'elles ne sont pas dans la forme de disciplines d'enseignement supérieur ou d'enseignement secondaire, comme les matières de l'école primaire, ou 1' " éducation à l'environnement"; mais elles soulèvent alors cet enjeu fondamental de pouvoir penser des actions a-disciplinaires du côté des élèves, et peut-être transdisciplinaires du côté des enseignants. En même temps, il ne faut pas oublier que les disciplines ont été construites pour former, pour « discipliner », et que, si elles ne relèvent pas d'une " essence éternelle », elles ont de très bonnes raisons d'être.

En réalité, le paysage pour les didactiques est sans doute encore plus brouillé et changeant : quelles sections diverses pour les thèses ou les habilitations, quelles trajectoires croisées vers quels faisceaux de compétences pour les personnes? Quels environnements et quels milieux pour travailler?

Pour revenir au master à la base de ces quelques réflexions, j'ai tenté de suggérer que le triplet «disciplinaire/pluridisciplinaire / interdisciplinaire » est nécessaire mais insuffisant : pour les enjeux, les problèmes et les décisions auxquels les didacticiens ont à faire face, il faut manifestement aussi le couple « a-disciplinaire / transdisciplinaire ». 


\title{
Commentaires sur le compte rendu
}

\author{
Jean-Claude MOUNOLOU, Professeur honoraire des universités, biologiste
}

Il y a trop longtemps que j'ai quitté la fac pour être vraiment pertinent. Voici donc des commentaires de second niveau :

- l'interdisciplinarité est parfois une question de convergence circonstancielle: soit les disciplines sont mûres en même temps et les affinités entre chercheurs font le reste, soit, parce qu'elles ne vont pas au même rythme dans leurs progrès, elles ne se rencontrent pas... Autre effet de circonstance : celui où une question interdisciplinaire est formulée, mais où les techniques pour en traiter ne sont pas prêtes. Tout cela peut compliquer l'enseignement ;

- l'interdisciplinarité ne me paraît enseignable que lorsqu'elle est reconnue et demandée par les enseignants au sens large et par la société. Sinon, c'est une affaire de rencontre d'individus, heureuse, mais difficile à faire accepter...

- l'interdisciplinarité guidée par l'aval et le pragmatisme. Oui, bien sûr, parce que l'emploi des étudiants est en jeu. C'est nécessaire mais cela peut s'essouffler et tourner à la pluridisciplinarité (les écoles d'ingénieurs qui ont une pratique ancienne le vivent, sans vouloir le reconnaitre souvent). Au plan intellectuel, cela demande de revenir souvent sur les accréditations des masters, mais c'est dur pour les enseignants qui risquent de se cabrer ou de rester derrière...

- enfin l'interdisciplinarité est à mon sens un élan temporaire et quand elle s'installe dans la routine ce n'est plus de l'interdisciplinarité ! Pour qu'elle soit intellectuellement vivante, il est sage de la questionner et la recommencer (souvent, mais à quel rythme ? le même pour tous ou non?).

Muriel MAILLEFERT, MCF, université de Lille 3, économiste

Les réflexions de Marcel Jollivet montrent bien la difficulté de passer d'une interdisciplinarité de proximité à une interdisciplinarité plus éloignée qui a comme barrière principale l'édification d'un langage commun et d'un socle commun de connaissances à partir desquelles chaque discipline peut communiquer avec les autres et se déployer dans son espace propre.

Néanmoins, il n'est pas anodin de remarquer que les exemples d'interdisciplinarité se trouvent le plus souvent dans les disciplines appliquées (ici les sciences de l'ingénieur) pour lesquelles la barrière de la connaissance pure est moins élevée. Néanmoins, l'interdisciplinarité est souvent, comme le souligne l'auteur, une simple juxtaposition, parfois hiérarchisée, de disciplines.

Il faut également souligner que, du point de vue de l'enseignement universitaire et de la recherche, on trouve des formations qui enseignent et défendent, et ce depuis assez longtemps, la pratique de l'interdisciplinarité. C'est le cas de la nébuleuse complexe des sciences sociales (économie-sociologie-histoire) - qui renaît dans certaines universités sous le vocable un peu surprenant d'humanités - et dont sont issus plusieurs générations d'enseignants et d'enseignants-chercheurs. Leur pratique de la pluridisciplinarité est une seconde nature, même si elle n'a pas été réfléchie ni théorisée : l'acculturation s'est faite très tôt. Néanmoins, il est clair que leur visibilité et leur reconnaissance est très faible dans le milieu universitaire qui préfère, y compris dans les universités de lettres et sciences humaines, la mono spécialisation. 


\title{
Témoignage des jeunes diplômés
}

\section{Initier aux approches interdisciplinaires dès le master : l'exemple du master 2 "Approches Interdisciplinaires du Vivant»}

\author{
Livio RIBOLI-SASCO, École normale supérieure et Elifsu SABUNCU, Institut Pasteur
}

L'interdisciplinarité prônée depuis déjà quelques années commence à faire son chemin, tant dans la pratique de recherche que dans la formation des jeunes, de l'école primaire aux études doctorales. Comme pour toutes les modes qui se propagent rapidement, la terminologie « interdisciplinaire» recoupe une diversité de pratiques d'enseignement et de recherche. Quelle que soit la définition que l'on en retienne, pour des apprentis chercheurs, une pratique de l'interdisciplinarité de qualité requiert une formation adéquate. On ne s'improvise pas interdisciplinaire. On peut considérer que, dans un contexte de compétition internationale accrue, une offre de formation à l'interdisciplinarité pourrait insuffler un surplus de dynamique à la recherche. Loin d'être une solution unique récusant les autres formations, celle-ci doit se concevoir en complément de l'offre existante.

Comme nous nous proposons de le montrer ici, la formation offerte par le master Approches Interdisciplinaires du Vivant (AIV), que nous avons suivie il y quatre ans en tant qu'étudiants et avec laquelle nous avons conservé des liens très forts, est une tentative de réponse à ces besoins, fondée sur un programme pédagogique rigoureux et intensif dont l'objectif est de stimuler la créativité par l'interdisciplinarité. Il est certes encore un peu tôt aujourd'hui pour évaluer globalement le succès d'une telle entreprise (elle a débuté en $2004)^{18}$. En ce qui nous concerne, alors que nous sommes actuellement en thèse et en postdoctorat, nous pouvons dire que cette formation a constitué pour nous un tournant dans nos parcours scientifiques.

\section{Quelles réponses apporter aux difficultés rencontrées dans les formations pour et par la recherche?}

Inventer aujourd'hui des formations interdisciplinaires en France doit se faire avant tout en prenant en compte l'état actuel des formations scientifiques et des difficultés que rencontre le monde de la recherche français. De ce point de vue, il nous semble nécessaire d'attirer l'attention sur deux points majeurs : 1. l'indispensable renforcement de la pratique de la recherche dans la formation universitaire ; 2 . le développement hautement souhaitable dès ce stade de la possibilité de s'ouvrir à l'interdisciplinarité.

1. En ce qui concerne la pratique de recherche, deux de ses aspects nous semblent insuffisamment pris en compte : la formation méthodologique et la formation à la gestion d'équipe et de projet.

- la formation méthodologique : le nivellement par le bas des moyens financiers et humains d'une bonne partie des formations universitaires françaises retarde l'apprentissage de la pratique de recherche ${ }^{19}$. Les étudiants entrant en master ne sont pas toujours entraînés à

\footnotetext{
${ }^{18}$ Il serait intéressant que des chercheurs étudiassent sur le long terme le parcours des étudiants qui auront été ainsi formés afin de passer d'un discours qualitatif à une solide approche statistique.

${ }^{19} 6820$ euros dépensés en moyenne par étudiant universitaire en 2003, à comparer aux 9750 euros dépensés en lycée. Source : ministère de l'Éducation nationale, de l'Enseignement supérieur et de la recherche. $18^{\mathrm{e}}$ place sur 23 dans l'OCDE en ce qui concerne le taux d'encadrement, 1 enseignant pour 18,1 étudiants. En additionnant les dépenses de R\&D et dans l'enseignement supérieur la France ne se place qu'au $10^{\mathrm{e}}$ rang dans l'OCDE avec $3,58 \%$ du PIB, contre plus de $5 \%$ pour la Suède, la Corée, les USA par exemple. Cours des Comptes, efficience
} 
analyser et critiquer un article publié dans une revue scientifique en anglais. Au moment où un étudiant doit rédiger son projet de thèse (fin du master 2), il est indispensable qu'il ait pu explorer de façon critique l'état des recherches dans le domaine recouvrant les questions qu'il souhaite aborder. Il est donc essentiel qu'il soit en mesure dès l'entrée en M 1 d'avoir un regard critique sur le travail de ses futurs collègues. Cette attitude d'analyse qui est pourtant à la base de l'activité de recherche est bien trop absente des formations dites "scientifiques" de l'école primaire à la licence. Les formations de niveau master, véritable apprentissage de la recherche, conditionnent la qualité des thèses ${ }^{20}$;

- formation à la gestion d'équipe et de projet : la recherche est un métier d'équipe, les savoirs se propagent rapidement, les idées émergent plus souvent autour d'un café que devant un écran d'ordinateur. Or les étudiants en fin de master sont peu préparés à intégrer une équipe de recherche; les formations à la gestion d'équipe, de projet ou de budget sont souvent absentes. Elles sont même dévalorisées aux yeux de certains car elles sont considérées comme « bassement» du domaine de l'économie ou du management. Pourquoi s'étonner ensuite du taux important d'abandon en cours de thèse $\mathrm{e}^{21}$, bien souvent lié à des problèmes relationnels avec les directeurs de thèses et les équipes? Pourquoi s'étonner de la difficulté de certains de ces étudiants à proposer en fin de thèse des sujets de recherche qui pourraient être financés ? La recherche doit rester un défi collectif, et cette démarche nécessite un réel apprentissage.

2. Renforcer la possibilité d'accéder à l'interdisciplinarité dès la formation universitaire passe par l'offre d'une ouverture sur les disciplines et l'apprentissage d'une autonomie pour en franchir les barrières. Il est fondamental de redonner une marge d'autonomie à l'étudiant. S'il est bien encadré, celui-ci est en mesure, en master, de s'aventurer dans les savoirs et les recherches, en se laissant mouvoir par ses seules passions et sa curiosité. Les étudiants se doivent certes d'acquérir des techniques pointues de recherche, mais ils se limitent trop souvent à travailler dans les seuls champs en permettant l'usage. Ils devraient plutôt se placer dans une optique de formation continue et d'acquisition de techniques en vue de répondre à une question posée. Nous considérons aussi qu'il n'est pas utile de simplement «élargir » le champ des possibles, en offrant par exemple plus de choix dans les options. Il faut bel et bien trouver de nouvelles formes de liberté à donner à l'étudiant et lui assurer une formation solide pour assumer pleinement cette autonomie. L'étudiant qui s'ouvre à l'interdisciplinarité doit acquérir des compétences indispensables à un travail fertile et ambitieux, et tout particulièrement : une souplesse de langage, car même si toutes les disciplines sont scientifiques, chaque science a ses subtilités de vocabulaire; la capacité à remettre en question sa propre démarche de recherche et à l'enrichir grâce aux interactions avec les différentes équipes de recherche, les encadrants et les autres étudiants; des compétences relationnelles, de la mobilité. Il doit aussi développer ses capacités à acquérir rapidement de nouveaux outils de recherche, avoir le courage de se laisser intéresser par des thématiques loin de ses domaines de prédilection, pour y trouver, parfois et par surprise, des résonances avec ses propres travaux.

Cette quête d'interdisciplinarité va aussi de pair avec une plus grande liberté dans le choix des thèmes de recherche. Répondre aux questions des jeunes et de la société : pourquoi

et efficacité des universités : observation récentes et nouvelles approches, communication à la Commission des finances, de l'économie générale et du plan de l'Assemblée nationale, décembre 2005.

${ }^{20}$ À cet égard la France a accumulé un grave retard sur la plupart des pays de l'OCDE. Ses thèses sont peu reconnues, elles débouchent sur des métiers peu rémunérés, elles n'entraînent que peu d'innovation technologique. En ce domaine, les difficultés et leurs causes sont nombreuses. Nous estimons toutefois qu'il ne faut pas négliger l'apport que pourraient avoir des thèses de qualité, imaginées au cours de master poussant à l'excellence et au renouvellement scientifique

${ }^{21} 40 \%$ des abandons en cours de thèse (jusqu'à $30 \%$ dans les disciplines de sciences dures et $65 \%$ en SHS) se font pour exercer un métier, la thèse ne semble donc pas valorisable professionnellement. Les déterminants de l'abandon en thèse : étude à partir de l'enquête Génération 98 du Céreq, P. Moguérou et al., février 2003. 
pas ? Le groupe embryonnaire à l'origine du master AIV était formé de personnes qui s'étaient tournées vers les sciences par engagement envers la société, ce qui peut sembler surprenant au premier abord. Les sciences et la recherche présentent aux yeux des jeunes deux points forts : la rigueur d'une démarche, l'innovation par l'exploration. Dans une société confrontée à des difficultés majeures comme celle du changement climatique, et qui se pose des questions sur son avenir, la recherche apparaît à certains comme une voix d'excellence pour apporter des solutions justes et argumentées à ces problèmes, qui les touchent en tant que citoyens. Par rapport à la politique, les sciences présentent à leurs yeux les avantages de la rigueur. Or, les cursus disciplinaires ne permettent pas d'aborder de grandes questions de société qui, par nature, se révèlent interdisciplinaires et qui restent pour autant pertinentes sur le plan scientifique.

En 2003, ce qui n'était alors qu'un groupe d'amis, familier des rencontres interdisciplinaires de Berder $^{22}$, composé de doctorants, étudiants de l'École normale supérieure en magistère, et de chercheurs passionnés par les recherches les plus récentes en biologie évolutive se retrouvait régulièrement pour discuter des dernières avancées et publications dans ce domaine. En critiquant des articles récemment publiés dans les revues les plus prestigieuses, en osant écrire à leurs auteurs pour questionner et proposer des développements aux recherches menées, les étudiants en master qui prenaient part aux travaux pendant leur temps libre ont acquis un savoir-faire scientifique complémentaire de leur formation disciplinaire. De cette expérience positive naquit l'idée que ce type de travail pourrait constituer une véritable formation.

Le petit club de discussion qui s'était entre temps élargi et discutait d'articles dans des domaines de plus en plus variés des sciences du Vivant a imaginé un véritable programme pédagogique en vue d'un master. Dans ce master, ils ont souhaité conserver l'ambiance de travail qu'ils avaient créée et mettre en œuvre les méthodes d'analyses et de questionnement qui avaient été développées de manière informelle. Le passage au LMD offrait l'occasion de restructurer et repenser les schémas pédagogiques. Ces étudiants et chercheurs ont alors proposé de créer un master 2 «Approches Interdisciplinaires du Vivant ».

\section{Le master 2 " Approches Interdisciplinaires du Vivant" (AIV)}

Le master AIV réunit une vingtaine d'étudiants qui ont en commun un intérêt pour le Vivant, mais qui ont des profils variés. Ils viennent des disciplines suivantes: biologie, mathématiques, physique, chimie, informatique et médecine. Le recrutement classique se fait après une première année de master. Des docteurs, post-doctorants, chercheurs et ingénieurs déjà titulaires d'un poste peuvent également profiter pleinement de cette formation à un moment où ils envisagent de changer de domaine de spécialisation ou s'ils souhaitent travailler à l'interface entre plusieurs disciplines. Le master accueille également des étudiants étrangers. Toutes les discussions lors des présentations, séminaires, clubs (voir ci-après) ont ainsi lieu en anglais, ce qui permet une meilleure interaction entre les étudiants des diverses nationalités, mais constitue également une préparation à la recherche scientifique dans un contexte international.

La grande originalité du master réside dans le déroulement de la formation qui y est donnée. Le parcours se déroule sur onze mois, de septembre à juillet. Il comprend: 1. un stage en résidence d'écriture de projet ; 2. trois stages de trois mois dans des laboratoires et la

\footnotetext{
${ }^{22}$ L' «École de Berder», du nom de la petite île du Morbihan où se déroule les rencontres, est une école de chercheurs, interdisciplinaire, d'échanges et de formation en biologie.

Cf. http://www.lptmc.jussieu.fr/users/lesne/berder2008.html. Exemples de quelques thèmes traités lors des précédentes éditions : Forme et information; Interactions et émergences; Le temps en biologie ; Information et optimisation; Biologie intégrative : L'organisation du vivant; Hasard, fluctuations et variabilité. En 2008, l'école se tiendra du 31 mars au 5 avril avec pour thème « Robustesse et adaptation en biologie ».
} 
soutenance des mémoires de stage; 3. des travaux bibliographiques; 4. l'organisation de groupes de discussion ; 5. la participation à l'École de Berder.

1. L'année débute, pour l'ensemble des étudiants de la promotion, les encadrants et plusieurs autres intervenants, par une semaine en résidence ${ }^{23}$. L'objectif est la sensibilisation à la démarche de recherche interdisciplinaire. Les activités de cette semaine s'articulent autour de plusieurs tâches : l'élaboration d'un projet de recherche par groupes de quatre étudiants ayant des formations disciplinaires différentes, des séances communes proposées par les encadrants dont le but est de stimuler un élargissement des champs de questionnement scientifique, et des discussions lors desquelles les chercheurs présents partagent leur expérience de recherche avec les étudiants.

La formation à l'écriture de projet se déroule sur plusieurs jours : les étudiants doivent se mettre d'accord sur une question qui met en œuvre des approches disciplinaires variées. Aucune contrainte n'est imposée quant aux thématiques abordées, aux moyens nécessaires ou à l'existence de travaux publiés traitant de la question. Les étudiants doivent faire preuve de curiosité et d'inventivité (pour trouver le sujet), de rigueur et de clairvoyance (pour la planification du protocole expérimental), d'habileté dans l'écriture, mais aussi de talents de communication et d'écoute (pour se comprendre par delà les formations initiales et malgré des différences de vocabulaire ou de méthodologie).

Les exercices proposés par les encadrants pendant des séances communes ont pour objectif d'aider les étudiants à ouvrir les yeux sur ce qui est parfois invisible à l'œil nonexpert ou... trop habitué. Diverses images, des figures d'articles scientifiques, des illusions d'optique, sont utilisées pour exercer le regard et montrer l'importance et la complémentarité des détails (zoom in, analogue d'une certaine façon à la spécialisation disciplinaire) et de la vue d'ensemble (zoom out, analogue à des approches transversales, intégratives entre disciplines) pour saisir les différents aspects d'un problème complexe.

Chaque soir, un chercheur travaillant à l'interface de plusieurs disciplines présente son parcours et ses recherches et discute avec les étudiants. Par son aspect informel, cette semaine de rentrée offre l'occasion aux étudiants de faire connaissance les uns avec les autres et avec les encadrants et chercheurs présents, d'établir des binômes de travail pour l'année, et d'apprendre à se connaître dans une ambiance détendue. Les étudiants sont également amenés à prendre conscience des différences de raisonnement associées aux différentes disciplines et aux difficultés de compréhension que cela entraîne, leur montrant ainsi des directions à approfondir lors de l'année à venir, pendant les stages, exercices et diverses activités.

2. Les étudiants choisissent trois laboratoires dans des disciplines différentes ou à l'interface entre deux disciplines, pour y mener un travail de recherche pendant chaque trimestre. Il s'agit pour eux de s'initier à la pratique du travail de recherche aux interfaces. Ils sont amenés à y utiliser les techniques les plus récentes et à questionner les nouvelles connaissances, les nouvelles avancées dans un domaine donné. Un au moins de ces stages doit être de caractère théorique et un autre de caractère expérimental. Ces stages peuvent avoir des sujets très différents, permettant ainsi à l'étudiant d'identifier un domaine qu'il souhaite approfondir pendant son doctorat. Ils peuvent aussi s'articuler autour d'une problématique commune, offrant ainsi trois approches complémentaires de la question. Ils sont l'occasion pour l'étudiant de s'immerger dans un domaine qui lui est inconnu, et de collaborer avec des personnes qui réfléchissent différemment, suivant leur discipline d'origine ou leur formation théorique ou expérimentale. Par ailleurs, il est important pour l'étudiant de bien choisir le sujet de sa thèse tout comme le laboratoire, et ces stages lui permettent de mieux se rendre compte des conditions dans lesquelles il devra travailler pendant les trois années de sa thèse. Chaque stage donne lieu à une présentation devant l'ensemble de la promotion, en anglais, ce

\footnotetext{
${ }^{23}$ Celle-ci se déroule dans la Station d'écologie expérimentale de l’École normale supérieure à Foljuif.
} 
qui constitue un réel exercice de communication des travaux de recherche à la communauté scientifique. Un étudiant qui souhaite approfondir un domaine particulier peut remplacer un de ces stages par des cours intensifs (par exemple un «cours Pasteur» ou un module à l'Université).

3. Chaque trimestre, les étudiants approfondissent une problématique actuelle de la recherche, en binôme interdisciplinaire, par des exercices bibliographiques et l'élaboration de projets. Il s'agit pour l'étudiant de s'exercer à l'analyse, à la critique et à la synthèse de travaux scientifiques, en vue de l'écriture d'un projet de recherche. L'exercice du premier trimestre consiste en la présentation critique d'un article récent paru dans un journal à peerreviewing. L'article doit être replacé dans son contexte; sa problématique et les enjeux qu'elle comporte doivent être mis en évidence; les résultats publiés doivent être finement analysés, en faisant preuve d'esprit critique quant à l'interprétation que les auteurs de l'article en donnent. Cet exercice a amené à plusieurs reprises des étudiants à prendre contact avec les auteurs des articles quand les explications fournies dans ces derniers ne leur paraissaient pas satisfaisantes. Lors du deuxième trimestre, de nouveaux binômes sont constitués et les étudiants travaillent sur une question scientifique controversée. Il s'agit cette fois d'un exercice de synthèse, où les étudiants doivent présenter l'état des recherches dans le domaine, identifier les différentes directions du débat, et dessiner les perspectives de développement des recherches. À cette analyse, s'ajoute la construction d'un projet de recherche qui permettrait de faire progresser la controverse. Enfin, au troisième trimestre, les étudiants doivent étudier et présenter un ouvrage scientifique de référence (au sens large du terme), et proposer un sujet de recherche qui en soit librement inspiré. Ce projet doit être rédigé sur le modèle d'une demande de financement. Il est ensuite soumis à la critique d'un binôme d'étudiants, dont les commentaires doivent être pris en compte par les auteurs du projet avant sa présentation.

Les présentations de ces travaux prennent la forme de discussions, lors desquelles les étudiants doivent convaincre leurs pairs de la pertinence de leurs questionnements et des moyens qu'ils proposent pour les résoudre. Trouver un vocabulaire commun entre chercheurs de disciplines initiales différentes n'est pas aisé et s'apprend d'autant mieux qu'on y est initié tôt. Les étudiants apprennent à dialoguer avec des collègues n'ayant pas eu la même formation qu'eux et acquièrent ainsi des connaissances, des concepts, des outils avec lesquels ils n'étaient pas familiers. La transmission de savoirs elle-même constitue un exercice très formateur ${ }^{24}$.

4. Au cours de l'année, les étudiants se retrouvent dans des «clubs», des groupes de discussion, qu'ils mettent en place autour de leurs intérêts communs. Le but est de développer leur esprit d'initiative, leur autonomie et leur curiosité. Le contenu des rencontres hebdomadaires, les articles à discuter ainsi que les chercheurs invités sont tous choisis par les étudiants. Le plus souvent, un étudiant ouvre la séance par la présentation du sujet en insistant sur les éventuelles controverses qui l'animent. Dans certains cas, c'est à un chercheur qu'est laissé le soin de cette introduction. Dans tous les cas, le débat est ensuite ouvert, avec la participation des étudiants et des chercheurs, à partir des questions soulevées par la présentation et par les articles lus par chacun. Les titres de quelques-uns des clubs des années précédentes donnent une idée de la diversité des sujets abordés, et de l'intérêt de mobiliser des approches interdisciplinaires : Merveilles de la Nature, Évolution, Maths-Bio, Bio-médecine, Protéines, Biophysique, Neurosciences, Biologie Synthétique, Flux et transferts, Morphogénèse. Les clubs sont également ouverts aux personnes extérieures au master. C'est ainsi que des personnes d'horizons très variés ont régulièrement participé aux clubs, apportant

\footnotetext{
${ }^{24}$ Sans surprise d'ailleurs, nombre d'entre eux s'investissent dans des actions de vulgarisation des sciences, telle la Fête de la science ou le festival Paris Montagne à l'ENS au mois de juillet.
} 
un réel élargissement aux discussions scientifiques et un regard critique supplémentaire sur les projets formulés par les étudiants.

5. Au cours de l'année, les étudiants prennent part à divers colloques, selon leurs centres d'intérêts personnels (par exemple aux Troisièmes entretiens de Bures, «Innovation dans les systèmes biologiques, mécanismes, évolution et conséquences", en décembre 2005 à l'Institut des hautes études scientifiques, ou encore au Séminaire de philosophie de la biologie, en 2006 à l'Institut d'histoire et de philosophie des sciences et techniques). Ils ont en outre l'opportunité de participer à l'École Interdisciplinaire de Berder, habituellement réservée aux chercheurs. Les étudiants ont l'occasion d'y échanger avec des chercheurs qui ont réussi à aller au-delà des barrières entre les disciplines et de participer à des conférences et des débats pointus. Les soirées sont également l'occasion de discussions plus informelles, prenant le prétexte d'un film, ou poursuivant les conférences de la journée. Le fait de passer une semaine sur une île avec 50 à 70 chercheurs, avec un rythme intense de conférences et des activités collectives (balades botaniques, ornithologiques, etc.) permet également aux étudiants de construire un réseau de connaissances dépassant celui des maîtres de stage ou encadrants de master, d'initier des collaborations, de discuter de projets. D'une façon générale, ces colloques et rencontres favorisent l'intégration des étudiants dans le milieu des chercheurs.

\section{Et après le master ?}

Un début d'évaluation de ce master peut être réalisé à partir du débouché des étudiants des deux premières promotions : plus de $90 \%$ d'entre eux sont actuellement en thèse. Certains choisissent d'effectuer leur thèse dans un laboratoire dont la discipline principale n'est pas leur discipline d'origine. Dans ce cas, l'étudiant est en mesure d'apporter des compétences propres à sa discipline d'origine à l'équipe du laboratoire d'accueil, et ayant acquis la capacité à collaborer avec des chercheurs d'une discipline différente, il élabore ainsi un projet original à l'interface entre plusieurs disciplines. Dans d'autres cas, l'étudiant reste dans sa discipline d'origine, mais est à même de mettre en place des collaborations avec des chercheurs d'autres disciplines pour renforcer la pertinence de son projet. Certains décident de réaliser leur thèse à l'étranger. L'intervention de plusieurs conférenciers étrangers favorise cette ouverture vers l'international, en donnant un aperçu de la recherche telle qu'elle se pratique à l'étranger, et en étant l'occasion de rencontres et de discussions enrichissantes.

Pendant cette année de master, les interactions des étudiants avec des personnes d'horizons très différents, ayant des centres d'intérêt très variés, favorise la découverte de nombreux domaines, et les étudiants s'orientent parfois dans des directions auxquelles ils n'auraient pas pensé auparavant. Ce master, par son ouverture, incite aussi les étudiants à s'investir dans des projets visant à développer les liens entre le monde de la recherche et la société.

Le dynamisme de ce groupe d'étudiants et de chercheurs a mené à l'élaboration de structures permettant de maintenir cette interaction scientifique fructueuse au-delà du master.

Dès 2004, les étudiants ont créé une association (Adefis) ${ }^{25}$ dont l'objet est la promotion et le soutien financier des approches interdisciplinaires lors des formations universitaires. En 2007, des étudiants ont participé au concours international de biologie synthétique iGEM $^{26}$ et ont remporté une médaille d'or devant des équipe d'université prestigieuses comme Harvard. En 2008, l'Adefis organise deux semaines de rencontres avec des élèves en master et doctorat chinois à l'Université de Pékin. L'Adefis avait également été une des force de proposition

\footnotetext{
${ }^{25}$ Association d'expérimentation en formation interdisciplinaire et scientifique, siège à l'ENS, 45 rue d'Ulm, 75005, Paris.

${ }^{26}$ International Genetically Engineered Machines.
} 
pour la mise en place d'une École doctorale expérimentale proposant ces nouvelles approches pédagogiques au niveau de la thèse. Cette école qui a ouvert en septembre 2007 sous le nom d'École doctorale européenne "Frontières du Vivant» regroupe des étudiants d'origine disciplinaire variée autour de thématiques nécessitant une combinaison de questionnements et de compétences et répond aux difficultés que certains étudiants issus de formation telle qu'AIV ont rencontrées pendant leur thèse.

\section{Conclusion : vers une indiscipline}

Le philosophe Jacques Rancière préconise «l'ignorance » du maître face à ces élèves. C'est ainsi que tous deux peuvent construire ensemble un savoir. Le maître est là pour guider dans la démarche, accompagner l'élève dans sa prise de risque face à l'inconnu qui est à maîtriser. L'interdisciplinarité que nous avons vécue et que nous proposons repose à l'extrême sur cette forme de rapport. C'est une aventure partagée entre étudiants de fin de master en quête de sujet de recherche et chercheurs toujours stimulés par l'exploration des nouvelles frontières de la connaissance. Ils avancent ensemble dans un dialogue interdisciplinaire où chacun incite et éclaire l'autre par ses compétences et ses savoirs. Au maître, l'avantage de l'expérience, de la pratique qu'il se doit de partager. La convivialité, le plaisir et l'amitié deviennent des valeurs de travail. Comme certains l'ont suggéré, cette forme d'interdisciplinarité tend à l'indisciplinarité, à une rébellion salutaire contre certains dogmes du fonctionnement universitaire, pour tirer parti au mieux des savoirs, de leur mutualisation, des campus et des interactions qu'ils offrent. Espérons que nos dirigeants politiques et universitaires sauront mener une stricte évaluation des formations pour voir l'intérêt éventuel de tels changements de paradigmes didactiques sinon scientifiques, pour les sciences et pour la société dans son ensemble.

\section{Remerciements}

Les auteurs tiennent à remercier chaleureusement Claire Ribrault et Amandine Véber, étudiantes d'AIV (promotions 2005-2006 et 2006-2007) pour leur précieuse aide dans la rédaction de cet article. Que soient également salués ici, avec toute la reconnaissance des étudiants du master AIV, les encadrants, intervenants et maîtres de stages qui permettent la réalisation de cette aventure au quotidien, et tout particulièrement Ariel Lindner et François Taddéi qui y ont investi beaucoup de leur énergie au cours de ces dernières années, en plus de leur activité de recherche. 


\section{Un panel de formations interdisciplinaires}

La majorité des exemples de formations interdisciplinaires qui sont cités ci-après sont ceux qui ont été présentés lors des Journées. Quelques-uns ont été ajoutés parce que les responsables s'étaient déclarés intéressés, mais étaient indisponibles et ne pouvaient donc pas participer aux Journées. L'intérêt de ces présentations détaillées est triple : bâties autour des questions générales qui organisent le compte rendu des Journées, elles leur donnent un contenu précis ; elles témoignent de la curiosité intellectuelle, de l'inventivité et du dynamisme, voire de l'enthousiasme, de collectifs d'enseignants et d'étudiants soucieux d'innover pédagogiquement pour répondre à de nouveaux besoins de connaissances : de ce point de vue, elles illustrent le rôle primordial du bricolage (ce « travail intellectuel non soumis à des règles théoriques » dont parle Levi-Strauss) dans l'innovation tant en matière d'enseignement que de recherche; et enfin, procédant d'un regroupement qui dépasse les particularismes des contenus thématiques, disciplinaires et institutionnels, elles constituent l'embryon d'un fonds d'archives - unique en son genre - fondé sur l'ambition commune de l'interdisciplinarité et lui donnant donc corps en tant que telle.

Ces expériences portent sur des masters. Ceci traduit l'idée - qui mérite d'être débattue selon laquelle accéder à une formation interdisciplinaire suppose une solide formation préalable dans une discipline et une certaine maturité intellectuelle. C'est là sans aucun doute une vision de la construction des connaissances qui est un héritage d'une certaine tradition universitaire. Mais c'est aussi, dans l'état actuel des choses, la seule réponse possible aux contraintes incontournables héritées de cette tradition. Une autre démarche, fondée sur une posture intellectuelle procédant d'une prise en charge initiale de la complexité des objets, des phénomènes et des questions, mériterait d'être explorée. Mais c'est là un autre chantier.

\section{Sciences de l'univers, environnement, écologie (Luc ABBADIE) ${ }^{27}$}

\section{Intitulé de la formation \\ Intitulé de la (des) spécialité(s) ou option(s)}

Niveau (master 1, 2)

Master Pro ou recherche

Responsable(s)

Date de création

Institution de rattachement

\author{
Master Sciences et technologies \\ Mention : Sciences de l'univers, environnement, écologie
}

Master 1 et 2

Il existe des parcours « recherche» et des parcours « recherche et professionnalisation »

Responsables : Alain TABBAGH et Luc ABBADIE
Campus Jussieu
Tour 56/66, $2^{\mathrm{e}}$ étage,
pièce 204, boîte courrier 210
4 place Jussieu
75252 - Paris Cedex 05

Tel : 0144275971

Mail : master.sduee@upmc.fr

Octobre 2004

Université Pierre et Marie Curie

\footnotetext{
${ }^{27}$ Les noms cités sont ceux des responsables qui ont validé la présentation de la formation interdisciplinaire correspondante.
} 
Institutions associées

Disciplines enseignées

\author{
Objectif(s) \\ de l'enseignement \\ (compétences \\ interdisciplinaires \\ recherchées)
}

\author{
Descriptif \\ de l'enseignement \\ (cours, TP, terrain, etc.)
}

Comment est organisée l'interdisciplinarité dans les enseignements?

\author{
Nombre moyen d'étudiants \\ (par année)
}

Paris 7, Paris 11, Université Versailles-St Quentin-en-Yvelines, Université Paris Est-Marne-la-Vallée, Museum national d'histoire naturel, École normale supérieure, INA-PG, École polytechnique, École nationale supérieure des télécommunications, École nationale supérieure des techniques avancées, Conservatoire national des arts et métiers, Observatoire de Paris

Climatologie, océanographie biologique, océanographie physique, sciences de la terre, hydrosciences, géotechnique, écologie, sciences de la biodiversité, organisés en sept spécialités.

La connaissance des milieux met en jeu des méthodes d'observation, d'analyse et de modélisation à des échelles spatio-temporelles variées. L'évaluation de la perturbation des milieux et de leurs conséquences repose sur l'observation et la modélisation de l'ensemble des aspects physiques, chimiques et biologiques du fonctionnement des systèmes environnementaux. Les métiers de l'environnement évoluent aujourd'hui vers une pluridisciplinarité accrue. L'objectif de la mention est de former des spécialistes capables d'analyser et de modéliser des situations complexes, à partir d'un bagage culturel suffisamment large pour une approche pluridisciplinaire effective et d'une maîtrise de savoirs et de savoirfaire plus spécialisés.

La mention couvre donc un spectre scientifique très large. Elle permet d'accéder à la complexité des champs de connaissances et à la diversité des débouchés tant dans le domaine de la recherche qu'au sein de l'entreprise. Les formations proposées sont directement associées à celles développées dans d'autres établissements franciliens.

Cours, travaux dirigés, stages de terrain, stages en laboratoire, stages en entreprises

Par nature l'orientation générale de la mention n'est pas disciplinaire, mais elle s'organise autour d'une perspective générale de connaissance des milieux dont dépend la (sur)vie des sociétés humaines. Une attention particulière a donc été apportée à l'organisation d'un tronc commun dans la perspective : - d'initier les étudiants aux grandes problématiques que posent les milieux naturels, leur évolutions passées, leurs futurs prévisibles ; - d'initier les étudiants à la démarche scientifique, de l'observation à la modélisation, leur donner les outils généraux en statistique, analyse et traitement des données ;

- de leur faire analyser par quelle(s) démarche(s) les sociétés humaines associent connaissances scientifiques et contraintes économiques et sociales dans des textes réglementaires qui bornent leurs actions et leur montrer quelles en sont les conséquences au niveau d'une entreprise.

260 étudiants par promotion 

Origines disciplinaires
des étudiants

Existe-t-il des échanges entre étudiants à l'occasion de travaux collectifs ?

Débouchés envisagés et/ou effectifs

Nombre d'enseignants

Statuts des enseignants (universitaire, chercheur, ingénieur, etc.)

Difficultés rencontrées

Propositions, pistes pour des améliorations

Sources
Étudiants titulaires d'une licence du domaine Sciences et Technologies en sciences de la terre, sciences de la vie, sciences physiques, chimie, chimie physique, mécanique et mathématiques appliquées.

La mention est également ouverte aux élèves des Grandes Écoles et des écoles d'ingénieurs possédant des compétences reconnues dans les domaines scientifiques et technologiques mentionnés.

Deux unités d'enseignement sont suivies par l'ensemble des étudiants toutes spécialités confondues : une UE sur les problèmes d'environnement, une UE de statistiques.

La mention Sciences de l'Univers-Environnement-Écologie (SDUEE) ouvre aux écoles doctorales et aux métiers de la recherche et de l'enseignement supérieur dans les disciplines de l'environnement que sont l'astronomie, l'océanographie physique, l'océanographie biologique, les géosciences, la climatologie, les sciences de la biosphère et des écosystèmes, l'écologie fonctionnelle et l'écologie de la biodiversité.

Insertion professionnelle : elle offre aussi des débouchés en matière d'instrumentation en environnement, de gestion de bases de données, de télédétection, de systèmes d'information géographique, de gestion des milieux marins et terrestres, d'aménagement du territoire, de réhabilitation des sites dégradés, de gestion et de valorisation de la biodiversité.

Environ 200

Universitaires, chercheurs

Certains enseignements interdisciplinaires ont du mal à recruter des étudiants pour des raisons d'accessibilité (les horaires sont incompatibles d'une spécialité à l'autre).

Mise en place de parcours hors spécialité, ou plus exactement à l'interface entre spécialités.

http://lmd.upmc.fr/baf.aspx?id=MSUE\&objet=f\&lang=fr

Et site de la mention : http://www.master.sduee.upmc.fr

\section{Logique, philosophie, histoire, sociologie des sciences (LoPHiSS Paris-Sorbonne) (Daniel ANDLER)}

Intitulé de la formation

Intitulé de la (des)

spécialité(s) ou option(s)

Niveau (master 1, 2)

Master Pro ou recherche
Mention : Philosophie et sociologie

Spécialité : LoPHiSS Paris-Sorbonne

(Logique, Philosophie, Histoire, Sociologie des sciences)

Deux parcours :

A : Philosophie et histoire des sciences ;

B : Philosophie de la connaissance et du langage

Masters 1 et 2

Master recherche 
Responsable(s)

Date de création

Historique

Institutions de rattachement

Institutions associées

Laboratoire(s) d'accueil

Disciplines enseignées
Responsable de la mention : Alain RENAUT

Responsable de la spécialité : Daniel ANDLER : professeur de philosophie des sciences et théorie de la connaissance (daniel.andler@ens.fr)

$1^{\text {er }}$ octobre 2005

Il existait à Paris I un DEA de philosophie et histoire des sciences et une maîtrise de logique, appuyés sur l'IHPST, tous deux interdisciplinaires et accueillant des étudiants de formation scientifique à côté des étudiants de philosophie. Il existait également à Paris 7 un DEA d'histoire des sciences, appuyé sur le REHSEIS, accueillant surtout des étudiants de formation scientifique (mathématiciens d'abord, puis physiciens et enfin biologistes). Paris IV offrait des compétences en épistémologie de tradition française, en philosophie analytique (philosophie des sciences, théorie de la connaissance, philosophie de l'esprit et du langage) et en philosophie des sciences sociales. Enfin, l'École normale supérieure a souhaité développer ces disciplines et fédérer les ressources parisiennes dans le domaine. D'où l'idée d'un master cohabilité par les quatre établissements.

Université Paris-Sorbonne (Paris IV), UFR de philosophie et de sociologie

Cohabilitation avec les universités Paris 1, Paris 7 et l'École normale supérieure (Ulm)

Les principaux à Paris IV :

- l'équipe d'accueil Rationalités contemporaines (EA 3559);

- le Centre d'études sociologiques de la Sorbonne.

Les principaux à l'extérieur de Paris IV :

- l'Institut d'histoire et de philosophie des sciences et des techniques (IHPST) (UMR 8590, CNRS - Univ. Paris 1, ENS) : 13, rue du Four, 75006 Paris ;

- l'Institut Jean-Nicod, UMR 8129 CNRS - EHESS, ENS. 1bis rue de Lowendal, 75007 Paris (en cours d'installation à l'ENS, 29, rue d'Ulm, 75005 Paris) ;

- le Centre de recherche en épistémologie et histoire des sciences exactes (REHSEIS, UMR 7596, CNRS-Université Paris 7);

- le Centre d'histoire des sciences et des philosophies arabes et médiévales (UMR 7062, CNRS - Univ. Paris 7, EPHE).

1. Histoire / sociologie des sciences ;

2. Philosophie des sciences lato sensu (incluant : logique philosophique, philosophie du langage, théorie de la connaissance);

3. Dominante disciplinaire (dans le cas d'un étudiant de philosophie : philosophie) ;

4. Formation scientifique complémentaire ;

5. Une unité dite d'initiation à la recherche / langue vivante.

Les « mastériens » inscrits à Paris IV auront ainsi accès, d'une part, à une très large gamme de cours de Paris 1 , Paris 7 , et l'ENS (ainsi qu'à certains cours du master de sciences cognitives ENS/EHESS/Paris 5), d'autre part à tous les enseignements de philosophie de Paris IV, dont évidemment ceux de philosophie des sciences (lato sensu), d'histoire des sciences, et enfin de sociologie. 


\author{
Objectif(s) de \\ l'enseignement, \\ compétences \\ interdisciplinaires \\ recherchées) \\ Descriptif de l'enseignement \\ (cours, TP, terrain, etc.)
}

\section{Comment est organisée l'interdisciplinarité dans les enseignements ?}

\author{
Nombre moyen d'étudiants \\ (par année)
}

\section{Origines disciplinaires des étudiants}

\section{Comment est prise en charge l'hétérogénéité des étudiants?}

Fournir les compléments nécessaires dans les disciplines nouvelles tout en renforçant considérablement les acquis dans la discipline d'origine.

M 1 : Les deux semestres, qui sont indépendants, se composent de 5 Unités d'enseignement (UE) chacun :

- UE1 Histoire ou sociologie des sciences

- UE2 Philosophie des sciences ou théorie de la connaissance

-UE3 Philosophie

- UE4 Formation complémentaire (sciences ou autre ; pour les agrégatifs, cours spécifiques à la préparation ou utiles pour le concours)

- UE5 Anglais langue de travail universitaire ou travail encadré de recherche encadré.

M 2 :

Le mémoire qui compte pour moitié dans l'appréciation de l'élève doit être conçu, tant par le choix du sujet que par la réalisation intellectuelle et matérielle, comme un véritable ouvrage de recherche, éventuellement publiable. Il est rédigé sous la responsabilité d'un professeur de Paris IV, qui appartient ou non à la spécialité LoPHiSS, et qui doit avoir donné son accord au projet. La seconde moitié se répartit entre 18 « unités de crédit européennes » à valider dans les séminaires de M 2 de la spécialité. Chaque semestre comprend 3 UE chacun, la troisième consistant dans le mémoire.

En M 1 et en M 2, l'élève est rattaché à un tuteur avec lequel, en début d'année, il établit son cursus personnel.

Les étudiants ont accès aux meilleures équipes de recherche, tant dans les spécialités philosophiques du secteur que dans des domaines interdisciplinaires en plein développement, en particulier les sciences cognitives (ils ont accès à certains enseignements du master de sciences cognitives ENS-EHESS-Paris V), les sciences sociales, l'environnement, la santé.

Sur les quatre établissements : M $1: 70$ étudiants M 2: 50 étudiants

Philosophie, mais aussi histoire, sciences sociales, sciences politiques, sciences de la nature, sciences biomédicales, juridiques et économiques, ou encore Grandes Écoles scientifiques ou commerciales.

Il est demandé de ne pas pratiquer la philosophie des sciences sans se familiariser avec une discipline scientifique particulière, mais cette familiarisation peut se faire pendant les années de master, et se poursuivre au cours du doctorat.

La pluridisciplinarité est organisée en M 1 en sorte que l'étudiant désireux de poursuivre en M 2 dans une autre spécialité ne souffrira pas d'un manque de préparation dans sa discipline principale. En effet, il aura accompli la moitié de sa scolarité dans cette discipline (pour la plupart des étudiants de Paris IV, la philosophie), ce qui représente, en enveloppe horaire, à peu près la totalité de l'horaire dans un M 1 monodisciplinaire. 
Existe-t-il des échanges entre étudiants à l'occasion de travaux collectifs ?

Débouchés envisagés et/ou effectifs

\section{Nombre d'enseignants}

\author{
Statuts des enseignants \\ (universitaire, chercheur, \\ ingénieur? etc.)
}

$Y$ a-t-il concertation entre enseignants? Sous quelle forme?

\section{Difficultés rencontrées}

Propositions, pistes pour des améliorations

Sources
Moins qu'il le faudrait. Cela dépend de la méthode de travail des professeurs animant un séminaire de M 2.

Les étudiants inscrits en seconde année du LoPHiSS peuvent s'inscrire aux préparations aux concours de recrutement, intégrer soit la recherche universitaire, soit toute une palette de professions à l'interface de la philosophie et des sciences et de la technologie et dans lesquelles leurs compétences philosophiques appliquées à un objet lié aux sciences seront de plus en plus appréciées.

Environ neuf à Paris IV. Plus d'une trentaine dans les trois autres établissements.

Universitaires, chercheurs

La spécialité fait partie de la formation cohabilitée LoPHiSS, coordonnée par les professeurs Jean Gayon (Paris 1) et Jean-Jacques Szczeciniarz (Paris 7). Les décisions pédagogiques communes sont prises par un conseil scientifique composé des coordinateurs et des professeurs ou directeurs de recherche. Mais l'essentiel de la coordination pédagogique se fait pour l'instant au sein de chaque établissement.

La mutualisation des enseignements, et la circulation des étudiants entre les différents établissements, sont limités par les règles et traditions propres à chacun. Il y a peu de réflexion commune, en profondeur, sur les conditions de l'interdisciplinarité, peu de mémoires ou stages co-dirigés, pas de séminaire ou de cours coenseigné (mais des cours « mosaïque » avec plusieurs intervenants).

Développer les activités communes (cours co-enseignés, mémoires co-dirigés, journées d'étude, défense publique générale des mémoires). L'internationalisation (accords avec centres étrangers) sera également un facteur positif.

http://www.paris-sorbonne.fr/fr/spip.php?article2962

\section{Développement et aménagement intégré des territoires (DAIT) (Patrick BLANDIN)}

Intitulé de la formation

Intitulé de la (des) spécialité(s) ou option(s)

Niveau (master 1, 2)

Master Pro ou recherche
- Évolution, patrimoine naturel et sociétés $(\mathrm{MNHN})$ pour le $1^{\text {er }}$ semestre

- Biologie, géologie, agroressources et environnement (Montpellier 2) pour le $2^{\mathrm{e}}$ semestre

Spécialité : Développement et aménagement intégré des territoires (DAIT)

Master 2

Master Pro 


\section{Responsable(s)}

Date de création

Historique

\author{
Institution(s) de \\ rattachement
}

Institutions associées

Laboratoire(s) d'accueil

\section{Disciplines enseignées}

Professeur Patrick BLANDIN, Muséum, UMR 5145 CNRS-Muséum, Département Hommes, Natures, Sociétés CP 135 57, rue Cuvier, 75005 Paris blandin@mnhn.fr Dr Arnaud MARTIN, MC Montpellier II Directeur du master BGAE Université Montpellier II, CEFE/CNRS 1919 route de Mende 34293 Montpellier Cedex 5 arnaud.martin@cefe.cnrs.fr

\section{4}

Formation créée en 1969, à l'initiative de la Commission française pour l'Unesco, comme cours post-universitaire destiné à des professionnels de pays en développement, avec la participation notamment des universités de Montpellier et de Toulouse, de l'École normale supérieure (Ulm) et de l'Institut national agronomique. La formation a été transformée en DESS au milieu des années 1980 (co-habilitation INA-PG/Paul Sabatier/Montpellier). Elle constitue le pilier de la première Chaire Unesco française, créée en 1994.

Muséum national d'histoire naturelle Université Montpellier 2

AgroParisTech (INA-PG)

- Chaire Unesco du développement durable, développement et aménagement intégré des territoires (coordination : Commission française pour l'Unesco ; responsable : Professeur. J.P. Prod'homme, AgroParisTech)

- Muséum national d'histoire naturelle : UMR 5145, UMR 5173

- Centre d'écologie fonctionnelle et évolutive, CNRS, Montpellier

- Sciences socio-économiques et développement

- Ethique environnementale et Droit de l'Environnement

- Anthropologie : savoirs locaux et biodiversité

- Sciences des territoires et des ressources naturelles (écologie des territoires - paysages, hydrosystèmes - géo-agronomie, biodiversité et biologie de la conservation)

- Systèmes d'Information Géographique

- Gestion de projets

La spécialité vise à former des professionnels de l'aménagement travaillant dans la perspective du développement durable, capables d'en aborder les aspects sociaux, économiques et écologiques de façon intégrée. Associant les sciences socio-économiques et les sciences des territoires et des ressources naturelles, les enseignements développent une approche interdisciplinaire des problèmes de développement. 


\section{Descriptif de l'enseignement}

(cours, TP, terrain, etc.)

\section{Comment est organisée l'interdisciplinarité dans les enseignements ?}

\section{Nombre moyen d'étudiants (par année)}

\author{
Origines disciplinaires \\ des étudiants
}

\section{Comment est prise en charge l'hétérogénéité des étudiants?}

\section{Existe-t-il des échanges entre étudiants à l'occasion de travaux collectifs ?}

\section{Débouchés envisagés et/ou effectifs}

1. Voyage d'études;

2. Formation théorique : sciences socio-économiques et développement, sciences des territoires et des ressources naturelles avec présentation théorique et pratique des systèmes d'information géographique (SIG), anthropologie, écologie, gestion des ressources naturelles ;

3. Formation pratique : gestion de projet et mise en situation, approfondissement sur les SIG et les bases de données environnementales, étude d'un problème d'aménagement d'un territoire en équipe pluridisciplinaire, proposée par une collectivité locale en concertation avec l'équipe pédagogique. Rapport d'étude réalisé collectivement.

Au plan méthodologique, l'accent est mis sur les techniques de travail en équipe de projet associant des compétences complémentaires. À cette fin, le stage professionnel est collectif, l'ensemble de la promotion s'investissant dans l'étude d'un problème concret d'aménagement. De façon générale, les enseignements sont organisés de façon à favoriser les échanges d'expériences entre étudiants venant d'horizons variés.

\section{4-18 dont 2/3 d'étrangers}

Le parcours proposé s'adresse plus particulièrement, à des étudiants étrangers, de préférence ayant déjà eu une première expérience de terrain et à des professionnels désireux d'approfondir leurs connaissances. Les étudiants français doivent de préférence avoir eu une expérience en pays en développement.

Les étudiants ont des formations initiales variées : biologie-écologie, géographie, sciences socio-économiques, écoles d'ingénieurs (sciences agronomiques, forestières).

Le voyage d'étude, en début de parcours, favorise la formation du groupe et les échanges informels, tout en donnant l'occasion d'un premier travail collectif (rapport de voyage). Les enseignements font largement appel à la pédagogie participative qui permet à chaque étudiant d'apporter et partager ses connaissances et expériences. Un séminaire est organisé où chacun présente un thème de son choix, en lien avec ses expériences de terrain. L'ensemble de la démarche pédagogique vise à valoriser la complémentarité des compétences et la reconnaissance mutuelle des spécificités, tout en faisant acquérir un socle commun de connaissances de référence.

Comme indiqué ci-dessus, ces échanges sont favorisés le plus possible.

Les étudiants étrangers, souvent déjà engagés dans la vie professionnelle, reprennent leurs fonctions ou évoluent vers de nouvelles responsabilités, en particulier dans les administrations et organismes chargés de l'aménagement et du développement. En Europe, les principaux métiers sont ceux de conseiller en développement local, de cadre technique en environnement, d'assistant à Maître d'ouvrage en environnement. Collectivités territoriales et bureaux d'études sont les principaux employeurs. 
Nombre d'enseignants

Statuts des enseignants (universitaire, chercheur, ingénieur, etc.)

$Y$ a-t-il concertation entre enseignants?

Sous quelle forme?

\section{Difficultés}

rencontrées

Propositions, pistes pour des améliorations

Sources
Un noyau d'une vingtaine d'enseignants et un nombre d'intervenants ponctuels variable.

Universitaires, chercheurs (CNRS, Inra, IRD ...), professionnels (AFD, Conseil supérieur de la pèche, bureau d'études, consultants...)

Une équipe pluridisciplinaire de pilotage pour la coordination générale (3-4 réunions annuelles), concertation « permanente » par mail.

Coordinations spécifiques par Unités d'enseignement.

Difficultés « administratives » pour la venue des étudiants étrangers (calendrier pour l'attribution de bourses, lenteurs pour l'obtention des visas, difficultés particulières pour les étudiants de plus de 26 ans), d'où des retards dans les arrivées, pouvant compliquer la mise en route de la dynamique de groupe.

Développer le partage d'une même " culture pédagogique » entre des intervenants nombreux, venant d'horizons divers

http://www.mnhn.fr/

http://w3b.info-ufr.univ-montp2.fr/siufr/offre

\section{Développement durable, management environnemental et géomatique (Catherine CARRE)}

Intitulé de la formation

Intitulé de la (des)

spécialité(s) ou option(s)

Niveau (master 1, 2)

Master Pro

ou recherche

Responsable(s)

Date de création

Historique

Institution(s)

de rattachement

Institutions associées

Laboratoire(s) d'accueil

Disciplines enseignées
Sciences humaines et sociales

Développement durable, management environnemental et géomatique

Master 2

Master professionnel en environnement, orientation maîtrise d'ouvrage

Catherine CARRE, MC à l'université de Paris 1, UFR de géographie, carre@univ-paris1.fr,

Mireille CHIROLEU-ASSOULINE, Professeur UFR d'économie Paris 1, Maryse DEGUERGUE, Professeur UFR de droit Paris 1

2005

Mise en place cadre réforme LMD

- École nationale des sciences géographiques (ENSG);

- Univ. Paris 1 : départements de géographie, de droit et d'économie

ENSPV (École nationale supérieure du paysage de Versailles)

Cerdeau, Ladyss, Prodig, Centre d'économie de la Sorbonne, Matis, Cogit

Droit, économie, géographie, paysage, SIG 


\section{Descriptif \\ de l'enseignement \\ (cours, TP, terrain, etc.)}

\section{Comment est organisée l'interdisciplinarité dans les enseignements ?}

Nombre moyen d'étudiants (par année)

\author{
Origines disciplinaires \\ des étudiants
}

\section{Comment est prise en charge l'hétérogénéité des étudiants?}

Existe-t-il des échanges entre étudiants à l'occasion de travaux collectifs ?

\section{Débouchés envisagés et/ou effectifs}

\begin{tabular}{|c|c|c|c|}
\hline $\begin{array}{c}\text { ENSG (École } \\
\text { nationale } \\
\text { des sciences } \\
\text { géographiques) }\end{array}$ & $\begin{array}{l}\text { Paris } 1 \\
\text { UFR de } \\
\text { droit }\end{array}$ & $\begin{array}{c}\text { Paris } 1 \\
\text { UFR d'économie }\end{array}$ & $\begin{array}{l}\text { Paris 1, UFR } \\
\text { de géographie } \\
\text { \& ENSPV }\end{array}$ \\
\hline $\begin{array}{l}\text { SIG Concepts et } \\
\text { applications } \\
\text { Conception } \\
\text { d'une base de } \\
\text { données } \\
\text { géographiques } \\
\text { Théorie des } \\
\text { graphes }\end{array}$ & $\begin{array}{l}\text { Droit des } \\
\text { collectivités } \\
\text { territoriales } \\
\text { Droit } \\
\text { spécifique } \\
\text { air, eau, } \\
\text { déchet, bruit } \\
\text { European } \\
\text { regulation } \\
\text { and } \\
\text { environment }\end{array}$ & $\begin{array}{c}\text { Stratégies } \\
\text { environnementales } \\
\text { des entreprises } \\
\text { Management } \\
\text { environnemental } \\
\text { Économie des } \\
\text { déchets et de l'eau } \\
\text { Économie des } \\
\text { ressources } \\
\text { naturelles }\end{array}$ & $\begin{array}{c}\text { Analyse et } \\
\text { gestion des } \\
\text { paysages } \\
\text { Les outils de } \\
\text { l'environnement } \\
\text { Les territoires } \\
\text { du risque } \\
\text { Mise en œuvre } \\
\text { d'une gestion } \\
\text { raisonnée des } \\
\text { territoires }\end{array}$ \\
\hline \multicolumn{4}{|c|}{$\begin{array}{l}\text { Séminaire commun Droit/Économie/Géogra } \\
\text { Préparation aux concours de la fonction territ } \\
\text { Atelier : réponse à une commande } \\
\text { Forum « Métiers de l'environnement » } \\
\text { Stage en entreprises ou collectivités }\end{array}$} \\
\hline
\end{tabular}

Une formation pluridisciplinaire et transversale Enseignements théoriques, méthodologiques et appliqués Stage en entreprises ou en collectivités territoriales

- Mise en situation des étudiants : stages, ateliers répondant à des commandes publiques ou privées ;

- Organisation d'un forum « Métiers de l'environnement» par les étudiants.

22 en 2005-2006, 23 en 2006-2007

Le master est une formation recrutant sur concours ouverte aux étudiants détenteurs d'une maîtrise ainsi qu'à ceux diplômés d'écoles d'ingénieurs. En 2006 : économie, gestion, géographie, géomatique, droit, économie internationale et de développement, IEP, management environnemental, biologie, management commercial.

Habitudes de formations pluridisciplinaires : master pro en aménagement, licence environnement, bi-deug géo-droit, géo-éco, géo-histoire...

- Atelier professionnel avec réponse à une vraie commande intégrant des étudiants des trois origines (droit, éco, géo) ;

- Organisation d'un Forum ;

- Travaux dans le cadre des modules (étude d'impact, analyse coûtbénéfice, gestion des risques).

- Ingénieurs subdivisionnaires de la fonction publique territoriale (après concours, préparation assurée dans le cadre du master) - Maîtrise d'ouvrage (Paysage, Géo-environnement, développement des territoires)

- Chargé de mission, écoconseiller auprès des collectivités territoriales

- Écoconseiller en entreprise

- Concours fonction publique territoriale 
Nombre d'enseignants

Statuts des enseignants (universitaire, chercheur, ingénieur, etc.)

Y a-t-il concertation entre enseignants? Sous quelle forme?

\section{Difficultés rencontrées}

Propositions, pistes pour des améliorations
17 enseignants universitaires +17 professionnels

Professionnels à $50 \%$ et enseignants spécialistes de l'environnement.

Réunion annuelle, à l'occasion évaluation formation par étudiants et réunion des enseignants dans les modules (un professeur responsable par module).

- L'interdisciplinarité dans les cours est plus une juxtaposition qu'une véritable interdisciplinarité sauf un séminaire commun ; mais là encore l'interdisciplinarité est résolue par des thèmes communs traités successivement par les trois disciplines

- Trouver chaque année des commandes réelles pour les étudiants

- Expérience récente

- Retard français par rapport aux pays du Nord où le cloisonnement ingénieur/sc. sociales est moins fort

- Perspectives d'un marché français de l'emploi très incertaines : pas de poste spécifique en DD

- Recrutement d'un PAST spécialisé en maîtrise d'ouvrage (porte de décloisonnement professionnelle), mutualisation des cours de sécurité en entreprise avec le master GGRC (gestion globale des risques) de Paris 1

- Renforcement du groupe de travail préparatoire pour le séminaire commun et les modules

\section{MASS - Modélisation et gestion sociale des risques (Cécilia CLAEYS-MEKDADE)}

\author{
Intitulé de la formation en \\ 2007
}

Parcours

Intitulé de la (des)

spécialité(s)

ou option(s)

Niveau (master 1, 2)

Master Pro ou recherche

Responsable(s) (titre(s), coordonnées)
MASS - Modélisation et gestion sociale des risques

MASS (Mathématiques appliquées et sciences sociales)

Représentations, modélisations et gestion sociale de risque

Master 1 et 2

Master Pro

Un master recherche est en cours de préparation, avec une ouverture vers la démographie et la géographie

Paul ALLARD, historien, professeur, département des Sciences humaines, université de la Méditerranée

Marie-Christine ROUBAUD, mathématicienne, UFR de mathématiques, université de Provence

Cécilia CLAEYS-MEKDADE, sociologue,

maître de conférences, université de la Méditerranée

Christine CAMPIONI, informaticienne, maître de conférences, université de Provence 
Date de création

Historique

Institutions de rattachement

Institutions associées

Laboratoire(s) d'accueil

Disciplines enseignées

Objectif(s) de

l'enseignement

(compétences

interdisciplinaires

recherchées)

Descriptif de l'enseignement (cours, TP, terrain, etc.)

Comment est organisée l'interdisciplinarité dans les enseignements ?

Nombre moyen d'étudiants (par année)
2005, poursuite au niveau $\mathrm{Bac}+5$ de l'ancienne maitrise MASS

Ce master prolonge une expérience originale de pluridisciplinarité menée au sein de la faculté des sciences de Luminy dans laquelle avait été créé il y a une trentaine d'années un département de sciences humaines et sociales. Longtemps pluridisciplinaire (cohabitation entre disciplines) la filière MASS est progressivement devenue interdisciplinaire (interpénétration scientifiques et pédagogiques), évolution notamment favorisée par l'implication d'une nouvelle génération d'enseignants-chercheurs issus de filières pluridisciplinaires.

Universités d'Aix-Marseille I (université de Provence)

et II (université de la Méditerranée)

Partenaires professionnels et spécialistes de la gestion du risque (collectivités territoriales, BRGM, INRST)

UMR Espace - DESMID, IML, LATP, LIF

-à l'UFR de l'université de Provence : Mathématiques appliquées ; Informatique

- à l'université de la Méditerranée : Sciences humaines et sociale ; Droit et Économie

Former des ingénieurs en sciences humaines et sociales capables de recueillir, traiter et analyser des données afin d'assurer la gestion sociale du risque dans des entreprises ou des collectivités territoriales

- $1^{\mathrm{re}}$ année : Enseignements de base en informatique, statistique et méthodologie de l'enquête (au $1^{\mathrm{er}}$ semestre), enseignements optionnels (à caractère méthodologique et thématique) (au $2^{\mathrm{e}}$ semestre), plus un mémoire de «mise en œuvre d'une recherche pluridisciplinaire $\gg$

- $2^{\mathrm{e}}$ année : Représentation et gestion sociales des risques, techniques de mise en lisibilité de données complexes, éléments de droit et d'économie pour la gestion du risque (Ier semestre), enseignements optionnels portant soit sur des techniques de traitement des données, soit sur des aspects économiques et juridiques de la gestion du risque, plus une préparation au monde professionnel à travers une formation à la « gestion et la réalisation d'un projet pluridisciplinaire »

Les thèmes des enseignements sont en grande majorité de nature méthodologique et technique. Ils sont très spécialisés et très précisément ciblés sur la thématique du master. Leur interdisciplinarité est donc dans leurs convergences analytiques. Par ailleurs, un des enseignements de base de la première année porte sur l'interdisciplinarité entre mathématiques et sciences sociales. Enfin, chacune des deux années comporte un exercice de formation à la conduite d'un travail pluridisciplinaire. Les mémoires interdisciplinaires sont encadrés par deux enseignants : un de mathématiques ou d'informatique et un de sciences sociales.

Entre 15 et 20 


\section{Origines disciplinaires des étudiants}

\section{Comment est prise en charge l'hétérogénéité des étudiants?}

\author{
Existe-t-il des échanges \\ entre étudiants à l'occasion \\ de travaux collectifs ?
}

\author{
Débouchés \\ envisagés et/ou effectifs
}

Nombre d'enseignants

Statuts des enseignants (universitaire, chercheur, ingénieur...)

$Y$ a-t-il concertation entre enseignants? Sous quelle forme?

\section{Difficultés rencontrées}

Propositions, pistes pour des améliorations
La plupart des étudiants proviennent d'une licence qui associe déjà sciences sociales et humaines et mathématiques : filière MASS avec trois parcours :

- mathématiques appliquées et sciences de l'homme et de la société, - mathématiques appliquées et économie,

- mathématiques fondamentales et sciences de l'homme et de la société), ces étudiants accèdent de droit au master MASS. L'acceptation d'étudiants d'autres origines disciplinaires se fait sur dossier, il s'agit principalement d'étudiants issus des filières mathématiques et informatique.

Bien que la mention MASS soit une dénomination nationale, le contenu des filières MASS varie significativement d'une université à l'autre, notamment en ce qui concerne les sciences humaines. Une mise à niveau de deux semaines est à ce titre proposée à l'ensemble des étudiants.

Les mémoires interdisciplinaires de M 1 et les projets pluridisciplinaires de M 2 sont le plus souvent réalisés en binômes. Les enseignements méthodologiques accordent une place importante au contrôle continu où le travail d'équipe est favorisé.

La gestion sociale du risque dans les entreprises et les collectivités territoriales. Une forte demande, mais encore peu formalisée, existe.

Une vingtaine, intervenant dans des proportions variées.

Universitaires, professionnels de la gestion du risque

Plusieurs enseignants partagent déjà la même culture scientifique, avec des spécialités différentes (histoire, mathématiques, informatique, sociologie,). Pluridisciplinaire, l'équipe pédagogique travaille en interdisciplinarité pour former des étudiants interdisciplinaires.

- Les préventions qui demeurent au sein de l'université contre la remise en cause des découpages disciplinaires au niveau des écoles doctorales et l'orthodoxie épistémologique qui va avec.

- Le blocage au niveau des débouchés académiques pour les étudiants dû au fonctionnement très disciplinaire des commissions de spécialistes.

- La concurrence pour les débouchés extra-universitaires avec les ingénieurs des Grandes Écoles.

Malgré les demandes existantes, l'ouverture vers la formation continue se révèle à ce jour difficile, faute de moyens logistiques et d'appui administratif. 


\section{Environnement, société : dynamiques et interfaces (Daniel DELAHAYE)}

\author{
Intitulé de la formation \\ Intitulé de la (des) \\ spécialité(s) ou option(s)
}

Niveau (master 1, 2)

Master Pro ou recherche

Responsable(s)

Date de création

Historique

Institution de rattachement

Institutions associées

Laboratoire(s) d'accueil

Disciplines enseignées

Objectif(s) de

l'enseignement

(compétences

interdisciplinaires

recherchées)

Descriptif de l'enseignement (cours, TP, terrain, etc.)
Master de Géographie

Environnement, société : dynamiques et interfaces

Master 2

Recherche

Daniel DELAHAYE

Courriel : daniel.delahaye@unicaen.fr

Laboratoire GEOPHEN

Esplanade de la paix, B.P. 5186, 14032 Caen Cedex

Tél. : 0231566594

Courriel : geographie.masters@unicaen.fr

Septembre 2004

La master a remplacé le DEA « environnement et sociétés »

Université de Caen Basse-Normandie, UFR de Géographie

MRSH (Maison de la Recherche en Sciences Humaines) de Caen.

CRESO (Centre de Recherche sur les Espaces et les Sociétés)

GEOPHEN (GEOgraphie PHysique et ENvironnement)

Discipline principale : Géographie

Discipline secondaires : histoire, sociologie, anthropologie

Étude des interrelations entre les sociétés et les espaces, avec une attention particulière pour les processus dynamiques aux interfaces et leur complexité, entre autres, dans une démarche d'analyse et de modélisation. Il s'agit ensuite de développer des orientations spécifiques sur les dynamiques environnementales et paysagères aux différentes échelles de temps, ou encore sur les mutations sociales et les interrelations entre les espaces urbains et ruraux.

L'enseignement, pour un total de 250 heures, se décompose en deux parties :

Premier semestre (170 h) :

Un tronc commun dispensé sur 2 ou 3 jours par semaine à raison de 8 semaines d'octobre à décembre :

- Dynamiques et interfaces : Concepts et méthodes de recherche ;

- Processus d'interface nature/société : Environnement et Risques ;

- Continuum urbain-rural : dynamiques et transformations de la structure sociale et fonctionnelle de l'espace ;

- Analyse et modélisation des systèmes complexes.

+ offre de conférences, de séminaires et de formations de l'École

doctorale.

+ offre de séminaires des pôles pluridisciplinaires de la MRSH

(Pôles Sociétés et espaces ruraux, données sociales, risques)

Second semestre $(80 \mathrm{~h})$ :

Les formations spécialisées dispensées aux $3^{\mathrm{e}}$ et $4^{\mathrm{e}}$ semestres sous la responsabilité de chacune des équipes de recherche d'accueil.

Celles-ci se divisent de la façon suivante :

- d'une part, des enseignements spécialisés en géographie physique et environnement et en géographie sociale, séminaires 
Comment est organisée l'interdisciplinarité dans les enseignements ?

Nombre moyen d'étudiants (par année)

Origines disciplinaires des étudiants

\author{
Comment est prise \\ en charge l'hétérogénéité \\ des étudiants?
}

Existe-t-il des échanges entre étudiants à l'occasion de travaux collectifs?

Débouchés envisagés et/ou effectifs

Nombre d'enseignants

Statuts des enseignants (universitaire, chercheur, ingénieur, etc.)

Y a-t-il concertation entre enseignants? Sous quelle forme?

Difficultés rencontrées

Propositions, pistes pour des améliorations

Source de laboratoires, ainsi que des travaux en groupe d'intégration à la recherche ;

- d'autre part, le projet personnel encadré par un enseignantchercheur HDR, sanctionné par un mémoire.

Participation au séminaires des pôles interdisciplinaires de la MRSH.

12

- Lauréats d'une première année de master avec la mention Bien ou Très Bien.

- Étudiants titulaires de diplômes de disciplines voisines (sciences humaines et sociales, sciences de la vie et de la terre, économie,...)

- Étudiants étrangers titulaires de diplômes équivalents

- La mise à niveau théorique se fait au premier semestre dans le cadre du module « Dynamiques et interfaces : Concepts et méthodes de recherche »

- La mise à niveau méthodologique et technique se fait dans le cadre des stages en laboratoire.

Pas formalisés dans le cadre de la maquette

Réalisation d'une thèse sur financement du ministère, de la région, du CNRS et sur contrat de recherche

24

Enseignants-chercheurs dont 11 habilités

La concertation se fait au sein des grands modules sous la forme de réunions de préparation des enseignements.

Les difficultés sont communes à tous les masters recherche :

- les master pro drainent la majorité des étudiants et souvent les meilleurs ;

- les masters recherche ne doivent pas être soumis aux mêmes règles $(\mathrm{H} / \mathrm{E})$ que les autres formations car il n'est pas cohérent de lancer 15 à 20 étudiants par année en thèse.

Dans la nouvelle maquette, nous envisageons un regroupement des filières pro et recherche. Cela va permettre de cibler l'offre de thèse sur quelques étudiants en fonction des financements tout en respectant les contraintes d'effectifs pour l'ouverture d'une formation.

Site Internet : www.unicaen.fr/ufr/geographie 


\section{Cogmaster (Emmanuel DUPOUX)}

\section{Intitulé de la formation}

\section{Responsable(s)}

Date de création

Institution de rattachement

Institutions associées

Laboratoire(s) d'accueil

Disciplines enseignées
Cogmaster

Emmanuel DUPOUX

dupoux@1scp.ehess.fr

Tél. : 0149542276

Tél. : 0144322351 (ENS)

http://lumiere.ens.fr/ cogmaster/www/

Secrétariat du master de sciences cognitives : EHESS

Alexia Chatirichvili - Bureau 245

54 boulevard Raspail

75006 Paris

Tél. : 0149542276

mastere@cogmaster.net

http://www.cogmaster.net

Direction des études : Franck Ramus

Tél. : 0144322356

etudes@cogmaster.net

Expérience d'enseignement interdisciplinaire de quinze ans

En co-habilitation EHESS, l'ENS et l'Université Paris Descartes

- mention «Sciences cognitives» du master recherche « Sciences sociales » (SS) à l'EHESS

- mention «Sciences cognitives » du master recherche « Sciences, technologie, santé » (STS) à l'ENS

- mention recherche «Sciences cognitives » du master «Sciences de la vie et de la santé » (SVS) à Paris 5

Il est en partenariat avec l'ENS-Cachan, l'École Polytechnique, l'ESPCI, l'Université Paris IV, l'Université Paris VI, l'Université Paris VIII et le RISC.

40 équipes d'accueil en région parisienne CNRS, INSERM, CEA

En première année de master (M1), la majorité des cours fondamentaux sera prise dans la discipline d'origine de l'étudiant afin de renforcer ses compétences de bases. Le reste des cours consistera en un tronc commun méthodologique, ainsi qu'en une introduction intensive aux concepts et outils nécessaires dans les autres domaines des sciences cognitives. Les enseignements fondamentaux, répartis en 5 'majeures', seront fournis par l'un des partenaires universitaires du master :

- majeure psychologie expérimentale avec Paris V;

- majeure neurosciences cognitives avec ENS, Paris V et Paris VI ;

- majeure philosophie et sciences sociales avec EHESS, ENS et Paris IV ;

- majeure modèles mathématiques et physiques avec Paris V

et ENS-Cachan ;

- majeure logique, linguistique et sciences de l'information avec ENS et Paris VIII.

Des stages de spécialités et mini-stages, au sein des laboratoires partenaires du master, viennent compléter ces enseignements. À l'issue de l'année de M 1, les étudiants devront avoir renforcé 
Niveau (master 1, 2)

Master Pro ou recherche

Objectif(s) de

l'enseignement

(compétences

interdisciplinaires

recherchées) leur discipline majeure à un niveau équivalent à celui d'un $\mathrm{M} 1$ monodisciplinaire. On attend en outre qu'ils aient assimilé les concepts, méthodes et enjeux centraux en sciences cognitives, et qu'ils connaissent les sources bibliographiques dans ce domaine. Enfin, que leurs connaissances leur permettent d'analyser et critiquer la littérature existante, afin d'élaborer un projet de recherche cohérent en vue d'une candidature en deuxième année de master (M 2).

En deuxième année de master (M 2), l'étudiant(e) sera confronté(e) aux thèmes de recherche actuels en sciences cognitives, et devra mener un travail de recherche dans l'un des laboratoires partenaires. Les sciences cognitives représentant une aventure intellectuelle d'une ampleur considérable, notre formation n'a pas la prétention d'englober toutes les approches possibles. Nous avons donc préféré nous concentrer sur les forces en présence, et sélectionner un petit nombre de domaines dans lesquels l'étudiant(e) bénéficiera d'un encadrement adéquat, dont est garante l'excellence scientifique de laboratoires d'accueil compétitifs au plan international.

Cette délimitation nécessaire est heureusement compensée par notre réseau international de partenaires européens, qui permettent aux étudiants du master d'accéder à des enseignements complémentaires dans les domaines laissés de côté.

Le champ couvert par le master correspond ainsi à ce qui représente la force principale des laboratoires présents dans notre programme ; nous l'avons divisé en 4 grands thèmes:

- perception et action ;

- langage et communication ;

- fonctions cognitives supérieures ;

- cognition sociale.

Ces thèmes, regroupant les cours avancés du master, permettent de proposer aux étudiants des Unités d'enseignement (UE)

véritablement multidisciplinaires, enseignant pour un même objet d'étude trois voire quatre approches méthodologiques différentes. Les majeures en M 2 n'existent plus que pour refléter la formation initiale de l'étudiant. Les cours, stages, et élèves sont censés être pluridisciplinaires.

\section{Masters 1 et 2}

Master recherche

L'enseignement du master s'appuie sur cinq principes :

1. Formation monodisciplinaire forte. Renforcement de la discipline initiale de l'étudiant, grâce à une sélection de modules parmi les cours offerts par les partenaires universitaires ;

2. Enseignement méthodologique solide. Acquisition des outils méthodologiques et conceptuels de base utilisés en sciences cognitives (protocoles expérimentaux, statistiques, méthodes d'imagerie cérébrale, programmation, etc.) ;

3. Ouverture interdisciplinaire ciblée. En master 2, offre de toute une série de séminaires interdisciplinaires, axés sur l'intégration multidisciplinaire ou ayant pour vocation d'explorer des thèmes émergents ;

4. Immersion rapide dans les programmes et les équipes de recherche. Les étudiants de $2^{\mathrm{e}}$ année sont intégrés à une équipe de recherche tout au long de l'année ; les étudiants de $1^{\text {re }}$ année sont 


\author{
Descriptif de \\ l'enseignement \\ (cours, TP, terrain, etc.)
}

\section{Comment est organisée l'interdisciplinarité dans les enseignements ?}

Nombre moyen d'étudiants (par année) Origines disciplinaires
des étudiants

Comment est prise en charge l'hétérogénéité des étudiants? incités à mener plusieurs stages ou mini-stages en laboratoire. Les étudiants sont ainsi entraînés à la rédaction de rapports scientifiques ainsi qu'à à leur présentation orale ;

5. Flexibilité. Chaque étudiant se voit désigner un tuteur, choisi au sein de l'équipe pédagogique du master. Celui-ci veille en particulier au choix de cours de l'étudiant, en l'aidant à sélectionner, parmi le large éventail d'enseignements monodisciplinaires et interdisciplinaires offerts tant par le master que par ses partenaires universitaires, ceux qui sont susceptibles de servir au mieux son projet scientifique.

Le but du master n'est pas de former des généralistes dont le discours engloberait l'ensemble des sciences cognitives, mais bien plutôt des scientifiques solides dans leur discipline. Il a pour but de former par la recherche à l'étude scientifique et interdisciplinaire des grandes fonctions mentales (perception, mémoire, raisonnement, langage, émotions, etc.).

- Pour les M 1 : l'objectif général poursuivi est le renforcement de la discipline de base de l'étudiant, accompagné par une ouverture interdisciplinaire aux sciences cognitives.

- Pour les M 2 : l'objectif général est l'ouverture interdisciplinaire et l'acquisition des outils nécessaires au stage long.

Le Cogmaster est une formation à l'intégration interdisciplinaire en sciences cognitives, c'est-à-dire à une démarche de recherche consistant à utiliser les outils de toute discipline possible qui permette d'éclairer une question scientifique particulière. Le principe d'ensemble est de consolider les bases disciplinaires de l'étudiant, en l'orientant vers l'acquisition d'outils propres à sa discipline de départ et ayant une interface avec les sciences cognitives. Dans cette première phase (principalement en M 1), l'étudiant sera introduit aux axes principaux des recherches en sciences cognitives. Dans un second temps (principalement en M 2), l'étudiant sera orienté vers les outils et disciplines complémentaires qui sont les plus importantes par rapport à son projet et ses intérêts scientifiques. En outre, l'année universitaire s'ouvre sur une quinzaine de mise à niveau. Elle est obligatoire et concerne aussi bien les étudiants de $M 1$ que de M 2. Cette quinzaine démarre avec une présentation générale de la formation et des cours proposés par le master ; elle permet de rencontrer les professeurs et tuteurs de la formation. Les autres journées ont pour objectif de présenter de façon accélérée quelques-uns des concepts et outils fondamentaux pour les sciences cognitives.

\section{M $1: 25$ \\ M 2:35}

Solide formation dans l'une des disciplines fondamentales des sciences cognitives : psychologie, biologie, mathématiques, physique, informatique, linguistique, philosophie, anthropologie, médecine et élèves ingénieurs des Grandes Écoles.

Les étudiants sont répartis dans des majeures en fonction de leur discipline d'origine. En M1, ils suivent ensemble un tronc commun, destiné à leur fournir des concepts et notions de base, puis un enseignement adapté à leurs connaissances. En M2, les cours sont 
Existe-t-il des échanges entre étudiants

à l'occasion

de travaux collectifs?

Débouchés

envisagés et/ou effectifs

\section{Nombre d'enseignants}

Statuts des enseignants (universitaire, chercheur, ingénieur, etc.)

Y a-t-il concertation entre enseignants?

Sous quelle forme?

\section{Difficultés rencontrées}

Propositions, pistes pour des améliorations

Source eux-mêmes interdisciplinaires, et les étudiants s'orientent en fonction de leurs intérêts spécifiques. Dans les deux années, nous organisons des cours de mise à niveau ou tutoriums, permettant aux étudiants de rattraper les notions qu'ils n'ont pas. Les tuteurs orientent les parcours individuels (choix des cours et des ministages).

Les ateliers et les tutoriums comportent des travaux en petits groupes (2 ou 3).

À l'issue de son master, l'étudiant(e) pourra postuler à l'une des allocations de recherche proposées par les Écoles doctorales associées au programme de master et en liaison avec le laboratoire où l'étudiant(e) souhaite faire son doctorat.

Plusieurs types de bourses et d'allocations permettent de mener à bien un doctorat à la sortie du master.

Une centaine

Chercheurs, enseignants chercheurs, anciens élèves,

Le master organise une réunion du Conseil pédagogique par an dont l'objectif est de faire le bilan et de proposer des restructurations au niveau global. Au niveau de chaque UE, le responsable réunit et coordonne les enseignants qui y participent.

L'hétérogénéité des niveaux en maths est difficile à gérer. Les autres hétérogénéités sont surmontables.

Cours miroirs (un cours pour matheux, un cours pour non matheux).

$\underline{\text { www.cogmaster.net }}$

\section{Vie, Terre, Environnement, Santé, Société (VTESS) (Patrick GIRAUDOUX)}

Intitulé de la formation

Intitulé de la (des) spécialité(s) ou option(s)

Niveau (master 1, 2)

Master Pro ou recherche

Responsable(s)
Sciences, technologies, santé

Spécialité : environnement, santé, société

Vie, Terre, Environnement, Santé, Société (VTESS) à partir de 2008

Master 1 et 2

Master à orientation recherche

(mais débouchés professionnels possibles)

Resp. pédagogique : Patrick GIRAUDOUX

tél. : 0381665745

fax : 0381665797

patrick.giraudoux@univ-fcomte.fr

Resp. pédagogique adjoint : Jean-François VIEL

tél. : 0381218738

fax : 0381668735

jean-francois.viel@ufc-chu.univ-fcomte.fr 
Date de création

\section{Historique}

Institution de rattachement

Laboratoire(s) d'accueil

Disciplines enseignées

\author{
Objectif(s) \\ de l'enseignement \\ (compétences \\ interdisciplinaires \\ recherchées)
}

Septembre 2000

Créé sous forme de DEA « Environnement, santé, société », le diplôme a été transformé en master en 2004.

Université de Franche-Comté

- Chronoenvironnement, UMR CNRS associée à l'Inra en janvier 2008 et regroupant les laboratoires de Biologie environnementale, Chrono Écologie, le département de Géosciences, Santé et environnement rural Franche-Comté et Physiopathologie, Épidémiologie, Résistance des agents infectieux

- Théoriser et modéliser pour aménager (UMR CNRS 6049)

- Biologie et gestion des pullulations (UMR Inra/IRD 1062)

- Laboratoire d'études et de recherches sur la rage et la pathologie des animaux sauvages, Agence française de sécurité sanitaire des aliments (AFSSA)

- Unité de recherches en technologie et analyses laitières (UP Inra)

- Mycotoxines et écotoxicologie terrestre (École nationale vétérinaire de Lyon, UMR Inra 123)

- Institut de recherche pour le développement (UR 024

Épidémiologie et prévention)

Écologie fonctionnelle, Biologie fonctionnelle, Génie de l'environnement, Géologie

- L'objectif prioritaire de cette spécialité est d'apprendre aux étudiants à partir d'un champ disciplinaire charpente à travailler avec des chercheurs de différents champs disciplinaires, à définir des problématiques scientifiquement et socialement pertinentes, et à participer à la mise au point d'approches pluridisciplinaires intégrées. En d'autres termes il s'agit, à partir du champ disciplinaire d'attache de l'étudiant renforcé en première année de master, de l'amener à combiner des connaissances et savoir-faire (actuellement éclatés entre disciplines) avec les besoins effectifs en recherche d'une société s'interrogeant sur l'environnement et ses dégradations. L'accent est notamment mis sur l'aptitude de l'étudiant à problématiser des questions scientifiques en tenant compte de la multiplicité des niveaux d'organisation et des échelles spatiales et temporelles organisant les processus des systèmes biologiques et géologiques.

- Les enseignements s'appuient sur des unités de recherche travaillant dans le domaine du diagnostic, de la diffusion et du devenir (de matière, d'énergie, de population, etc.) dans la biogéosphère à tous les niveaux d'organisation biologique et géologique et de l'impact de ces systèmes sur la santé. Ils s'appuient notamment sur l'organisation thématique de l'UMR

Chronoenvironnement support principal de la formation.

- Les unités de recherche du master cherchent à comprendre l'intégration spatiale, temporelle et fonctionnelle des composantes des systèmes environnementaux en question et participent à des grands projets internationaux dans ce domaine. 


\section{Descriptif \\ de l'enseignement}

(cours, TP, terrain, etc.)

\section{Comment est organisée l'interdisciplinarité dans les enseignements ?}

\section{Nombre moyen d'étudiants (par année)}

\author{
Origines disciplinaires \\ des étudiants
}

\section{Comment est prise en charge l'hétérogénéité des étudiants?}
Existe-t-il des échanges entre étudiants à l'occasion de travaux collectifs ?

Les champs disciplinaires charpente sont organisés au sein du M 1 en plusieurs parcours :

- Écosystèmes et environnement (UFR ST) ancre les étudiants dans les domaines écophysiologiques, populationnels et écosystémiques du corpus ;

- Biologie environnementale (UFR ST) ancre les étudiants dans les domaines moléculaires, cellulaires et éco-physiologiques du corpus ; - Géologie (UFR ST) ancre les étudiants dans les domaines relatifs aux écoulements, déformations et transferts, et les applications ; - Sciences de la vie et de la terre pour l'enseignement (UFR ST) qui prépare les étudiants aux concours de l'enseignement ;

- Génie de l'environnement (UFR STGI) ancre les étudiants dans les domaines liés au génie des procédés de l'environnement.

Les étudiants peuvent, par leur choix d'unité, moduler ces parcours types et les recomposer ou en additionner les composantes.

Ces parcours donnent accès, en deuxième année, aux spécialités VTESS et à d'autres spécialités de master.

Une attention particulière est portée sur les stages et écoles terrain à la fois en M 1 et M 2. Ceux-ci conduisent l'étudiant en M 1 à bénéficier d'une expérience en entreprise ou/et en unité de recherche universitaire (ex. unité Travaux d'étude et de recherche). Il bénéficie également d'occasions multipliées de mise en situation (première école terrain en début d'année universitaire, puis unités optionnelles dont la plupart incluent une école terrain au deuxième semestre). L'étudiant peut donc progressivement affiner ses choix de spécialité sur cette base.

La spécialité VTESS inclut en deuxième année une école terrain d'intégration pluridisciplinaire dès le premier semestre, et un stage de recherche plein temps au deuxième semestre du M 2, l'accueil en situation étant réalisé à temps partiel (hors enseignements) dès l'inscription en M 2.

Chaque module de deuxième année est piloté par des responsables pédagogiques appartenant à deux disciplines différentes, les exercices et sujets d'examens portent une dimension intégrative et pluridisciplinaire importante.

En deuxième année de la spécialité : 15-20 étudiants

Étudiants de toutes les disciplines impliquées (biologistes, géologues, archéologues, sociologues, médecins, pharmaciens...) ayant totalisé 30 crédits d'une première année de master dans ces disciplines ou titulaires d'une maitrise (ou d'un diplôme équivalent).

À travers l'existence d'un tronc commun important et d'écoles terrain intégratives où les synergies entre disciplines sont recherchées.

Oui 


\section{Débouchés envisagés et/ou effectifs}

Nombre d'enseignants

Statuts des enseignants (universitaire, chercheur, ingénieur, etc.)

$Y$ a-t-il concertation entre enseignants?

Sous quelle forme?

Difficultés rencontrées

Propositions, pistes pour des améliorations

Source
108 diplômés de 2000 (date de création de la spécialité) à 2007 (M : $46 \%, \mathrm{~F}: 54 \%$ ).

Suivi 6 mois après date d'obtention du diplôme (dernière promotion prise en compte : 2006) :

Études doctorales dans le cadre

d'un contrat salarial (allocation, etc.) $\quad 57 \%$

Professionnel sciences médicales

et pharmaceutiques $\quad 12 \%$

Chargé de mission environnement $\quad 9 \%$

Autres situations professionnelles $\quad 8 \%$

Réorientation $\quad 1 \%$

Recherche d'emploi $\quad 1 \%$

Abandon $\quad 1 \%$

Ajourné $\quad 1 \%$

Pas de réponse $\quad 9 \%$

40

Enseignants-chercheurs universitaires et chercheurs CNRS, Inra, etc.

Essentiellement en comité de pilotage, et en co-pilotage des unités.

- Se forger une culture et des pratiques communes prend du temps !

- Double coordination pédagogique des modules.

- Nécessité de trouver des enseignants ayant une pratique de recherche pluridisciplinaire au plus haut niveau réelle.

- Compartimentation du système d'évaluation de la recherche en

France (DS, CNU, sections, etc.), des structures internes aux universités (UFR, etc.).

- Hétérogénéité des pratiques pédagogiques et des modes d'évaluation entre sciences biologiques et géologiques / sciences humaines et sociales.

-Évolution démographique des formations « recherche » en rapport avec l'attractivité décroissante des métiers de la recherche.

Les éléments structurels évoqués dans la cellule plus haut sont actuellement profondément figés. La seule latitude de manœuvre est locale et interne à l'organisation pédagogique du diplôme. L'attractivité décroissante des métiers de la recherche incite à la conception de masters intégrés pro/recherche.

http://sciences.univ-

fcomte.fr/formations/descriptif/masters/environnement.htm 


\section{Espaces, sociétés rurales et logiques économiques (ESSOR) \\ (Anne-Marie GRANIE)}

Intitulé de la formation

Intitulé de la (des)

spécialité(s) ou option(s)

Niveau (master 1, 2)

Master Pro ou recherche

Responsable(s)

Date de création

Historique

Institutions

de rattachement

\section{Laboratoires}

\section{Disciplines enseignées}

ESSOR (Espaces, sociétés rurales et logiques économiques)

Domaine : Sciences humaines et sociales

Mention : Géographie et aménagement

Spécialité : Ruralités et dynamiques territoriales

Master 2

Master recherche

Laurien UWIZEYIMANA

uwizeyim@univ-tlse2.fr

1991 par des géographes, économistes et sociologues

Tutelles à l'origine : Université Toulouse le Mirail (UTM), Institut national polytechnique de Toulouse (INP), École nationale de formation agronomique de Toulouse-Auzeville (ENFA). Organisation dès les années 1990 d'un doctorat de $3^{\mathrm{e}}$ cycle de formation à la recherche centré sur la ruralité.

1. UTM, département géographie-aménagement, UFR sciences, espaces, sociétés

2. ENFA, département Économie, sociologie du développement

3. INP /École nationale supérieure agronomique de Toulouse

(ENSAT), département Sciences économiques. et sociales

Laboratoire d'accueil : Dynamiques rurales (UTM, ENFA, INPENSAT)

Directrice : Anne Marie GRANIE (anne-marie.granie@educagri.fr) ; directrices adjointes Hélène GUETAT (guetat@univ-tlse2.fr)

et Geneviève N'GUYEN (n'guyen@ensat.fr)

Laboratoires associés :

- Certop - « Travail, Organisations, Pouvoirs » UTM

- Etiq - « Emploi Innovation Compétence » Inra Toulouse

- Agir - Inra Toulouse

- « Intervention publique, espaces, sociétés », IRD/CNRS, Maison

des Suds à Bordeaux

- « Aménités et Dynamiques de l'Espace Rural » Ader, Cemagref

à Bordeaux

- «Architecture, paysages, sociétés et modèles » MAP/ASM-CNRS

à Toulouse

- Autres collaborations : Cirad - CNEARC (Centre national d'études agronomiques des régions chaudes) à Montpellier

Géographie, sociologie, économie, développement rural.

La formation s'articule autour des thématiques suivantes :

- production et gestion des ressources dans les espaces ruraux, au travers des filières agro-alimentaires, la qualité des produits, le développement durable ;

- nouvelles fonctions et organisations de l'espace : logiques territoriales, ruralités émergentes, modes d'habiter, institutions territoriales, formation et compétences des acteurs du développement, rapports de genre. 


\author{
Objectif(s) de \\ l'enseignement \\ (compétences \\ interdisciplinaires \\ recherchées) \\ Descriptif de \\ l'enseignement \\ (cours, TP, terrain, etc.)
}

\section{Comment est organisée l'interdisciplinarité dans les enseignements ?}

Nombre moyen d'étudiants (par année)

\author{
Origines disciplinaires \\ des étudiants
}

\section{Comment est prise en charge l'hétérogénéité des étudiants?}

Les compétences acquises concernent la recherche scientifique applicable également à la recherche-action.

40 heures de cours +85 heures d'encadrement collectif

M 1 - Simulation pratique des premières étapes d'une recherche

M 2 - Collecte et traitement de l'information

M3 - Traitement et analyse statistique des données

M4 - Epistémologie de la recherche et construction du mémoire

L'interdisciplinarité est présente dans des situations concrètes:

- dans l'explication de l'histoire de la construction de la discipline :

par exemple en montrant comment la géographie a emprunté à la sociologie le concept de représentation sociale ;

- l'exigence de travailler à partir de l'empirie d'objets de recherche mobilisés durant les cours : notamment les identités territoriales (communales), la question des limites socio-spatiales, le développement. On mobilise alors nos travaux de chercheurs engagés dans l'interdisciplinarité (socio-géographie), tout en insistant sur l'importance de l'affirmation des champs au sein de la discipline ;

- les manières d'expliquer comment on construit nos objets : sur l'approche genre, l'approche territoriale et identitaire par exemple ; - les méthodologies utilisées et explicitées : l'approche compréhensive (comme la co-construction de la rencontre), l'écriture filmique (par exemple, interrogation sur le sens du lieu, sur la manière de prendre en considération l'émotion, les gestuelles, comment filmer les récits de pratiques ou d'itinéraires de vie) ; - partir des savoirs situés des étudiants : comment interroger leurs expériences, leurs témoignages de situations ;

- le dispositif de formation :

- les séminaires propres à la formation doctorale et ceux de la MSH sont des lieux d'échanges inter laboratoires, notamment avec des chercheurs en audio-visuel. Entre la sociologie et la géographie des univers communs unissent les approches et les réflexions; le cinéma ouvre à des langages différents : la prise en compte de l'esthétique, de l'émotion, du verbe ; - les co-encadrements et les comités de thèse permettent une réelle interactivité qui nourrit une co-construction permanente.

25

- Des étudiants en formation initiale ayant obtenu un niveau de diplôme $\mathrm{Bac}+4$ minimum (priorités aux géographes, agronomes, économistes, sociologues et, de manière complémentaire, aux juristes).

- Des professionnels des organismes d'aménagement et du développement territorial.

- Des étudiants en formation continue.

Les travaux par groupe sont effectués dans une configuration interdisciplinaire et interculturelle. 


\section{Existe-t-il des échanges entre étudiants \\ à l'occasion \\ de travaux collectifs ?}

Débouchés envisagés et/ou effectifs

\section{Nombre d'enseignants}

Statuts des enseignants (universitaire, chercheur, ingénieur, etc.)

Y a-t-il concertation entre enseignants? Sous quelle forme?

\section{Contraintes et atouts}

\author{
Propositions, pistes \\ pour des améliorations
}

\section{Source}

La simulation de recherche (une semaine de stage de terrain avec évaluation orale et écrite) donne l'occasion aux étudiants de construire de l'interconnaissance et de confronter leurs systèmes de représentations scientifiques.

-Les concours d'entrée dans l'enseignement supérieur. - Les organismes et instituts de recherche tels que le CNRS, l'Inra et l'IRD, le Cirad.

- Les services privés, publics de l'aménagement et du développement agricole et rural, nationaux et internationaux, et les collectivités territoriales.

\section{5}

Enseignants-chercheurs des trois établissements + chercheurs Inra, CNRS et des conférenciers

Ingénierie de formation, co-encadrement de mémoire, séminaires thématiques, travaux de recherche et d'écriture

-La visibilité de la formation doit être renforcée. Elle reçoit de plus en plus de demandes d'étudiants en reprise d'étude (inscrits dans des parcours professionnels dans les métiers du développement, des travailleurs sociaux, d'une activité professionnelle agricole, d'administrateurs, de personnels d'ONG, etc.), notamment des pays du Sud.

-L'interdisciplinarité est recherchée pour résoudre des questions d'ordre pratique. Elle est opérationnelle pour rendre compte de la complexité des objets d'études. Elle est appréciée par le placement de nos étudiants, en regard de leurs compétences à comprendre des objets de recherche complexe en mobilisant des pluri regards et aussi en mobilisant des méthodologies appropriées. L'interdisciplinarité correspond à une demande sociale rendue lisible par la constitution des pôles de compétences régionaux (par exemple à Toulouse autour de la production et de la consommation de produits alimentaires, du territoire et de la santé). En revanche, elle apparaît plus " périlleuse » pour de plus jeunes étudiants souhaitant s'inscrire dans un projet de concours de l'enseignement supérieur (voire de la recherche) en raison de l'appréhension du passage de la qualification auprès du CNU.

-Insuffisance du temps de la réflexivité individuelle et collective. -Difficulté de positionnement entre disciplines (hiérarchisation) et positionnement de l'identité professionnelle de l'enseignantchercheur.

Conduire une réflexion action sur les avancées de la recherche avec l'interdisciplinarité pour mieux maîtriser l'interdisciplinarité dans l'enseignement des contenus et des méthodes. L'objectif étant de former à la compréhension de la complexité.

http://www.ensat.fr/NosFormations/MasterEssor.htm 


\section{Ingénierie et management de l'environnement et du développement durable (IMEDD) (Bertrand GUILLAUME)}

\author{
Intitulé de la formation \\ (titre)
}

Niveau (master 1, 2)

Master Pro ou recherche

Responsable(s)

Date de création

Historique

Institution de rattachement

Institutions associées

Laboratoire(s) d'accueil

Disciplines enseignées

Objectif(s)

de l'enseignement

(compétences

interdisciplinaires

recherchées)

Descriptif de

l'enseignement

(cours, TP, etc.)

Comment est organisée

l'interdisciplinarité

dans les enseignements ?

Nombre moyen d'étudiants (par année)

Origines disciplinaires des étudiants

\section{Comment est prise} en charge l'hétérogénéité des étudiants?
Ingénierie et management de l'environnement et du développement durable (IMEDD)

Master 1 et Master 2

Historiquement master pro, mais distinction obsolète

Bertrand GUILLAUME, Ing., PhD, MC

UTT-CREIDD, 12 rue Marie Curie, 10000 Troyes

2004 (en instance de renouvellement par le ministère)

2001 : DESS Ecologie industrielle

2004-2007 : Master MEDD

Université de technologie de Troyes

Université de Sherbrooke (Canada) ; Université de Lausanne (Suisse) ; Université polytechnique de Turin (Italie)...

Centre de recherches et d'études interdisciplinaires sur le développement durable (CREIDD)

Technologie, management, sciences de l'homme et de la société

Ingénierie et gestion de projet, appliquées à des stratégies de dématérialisation des activités économiques des entreprises et des territoires (écologie industrielle, éco-conception, mise en œuvre raisonnée d'écotechnologies)

Cours, travaux dirigés, travaux pratiques, séminaires, visites, projets individuels et collectifs, stages

- Directement, dans les enseignements d'intervenants professionnels et académiques issus d'horizons différents et placés sous la responsabilité d'enseignants-chercheurs ayant déjà une culture interdisciplinaire

- Indirectement, par un enseignement appelant des synthèses, des recompositions et des arbitrages autonomes ( $c f$. supra)

24

Recrutement varié (ingénieurs, universitaires en sciences et techniques, mais aussi diplômés en sciences politiques, géographie, économie et sciences humaines...) en formation initiale ou continue ; recrutement et ouverture ambitieuse à l'international (recrutements, mobilité...)

Activités de travaux dirigés, jeux de rôle et séminaires, projets en petits collectifs, encadrement varié de l'équipe 


\author{
Existe-t-il \\ des échanges \\ entre étudiants \\ à l'occasion \\ de travaux collectifs? \\ Débouchés envisagés \\ et/ou effectifs
}

\section{Nombre d'enseignants}

Statuts des enseignants (universitaire, chercheur, ingénieur, etc.)

$Y$ a-t-il concertation entre enseignants ?

Sous quelle forme?

\section{Difficultés rencontrées}

Propositions, pistes pour des améliorations

Source
Oui, avec des résultats très intéressants :

- gain de capacités d'adaptation et de questionnements des étudiants ;

- mise en perspective des compétences disciplinaires ;

- reconstruction prometteuse des schémas de pensée...

- Cadres et décideurs du secteur public ou privé

- Gestionnaires de systèmes socio-techniques innovants

- Chargés de mission développement durable

- Responsable environnement / éco-conception /énergie

- Thèse de doctorat

Une dizaine de permanents + vacataires extérieurs

Enseignants-chercheurs, chercheurs, ingénieurs, le plus souvent avec une double formation

Oui : réunions pédagogiques et pilotage en petite équipe interdisciplinaire appuyée sur un laboratoire de recherche

- Articuler transmission de savoir-faire spécifiques et développement de capacités génériques des étudiants (personnelles, managériales, critiques...)

- Assurer un socle commun de connaissances malgré la diversité des profils, et promouvoir dans le même temps une souplesse suffisante pour assurer le développement de projets professionnels spécifiques. - Poursuivre l'ouverture des enseignements à des développements prospectifs/aux frontières de la recherche avec les contraintes évoquées ( $c f$. supra).

- Poursuivre l'articulation des connaissances par l'approche systémique et densifier le tutorat.

- Promouvoir l'innovation pédagogique en conciliant autonomie des étudiants et encadrement proche.

www.utt.fr/medd

\section{1. Économie et gestion de l'environnement et du développement durable (Muriel MAILLEFERT)}

Intitulé de la formation en 2007

Intitulé de la (des) spécialité(s) ou option(s)

Niveau (master 1, 2)

Master Pro ou recherche

Responsable(s)

Date de création
Économie et gestion de l'environnement et du développement durable

Ce master est lui-même une spécialité du master « Économie et gestion des organisations »

Master 1 et 2

Master Pro

Muriel MAILLEFERT, économiste, MCF, université de Lille 3 2004 


\section{Historique}

Institutions de rattachement

Institutions associées

Laboratoire(s) d'accueil

Disciplines enseignées

Objectif(s) de

l'enseignement

(compétences

interdisciplinaires

recherchées)

Descriptif de l'enseignement

(cours, TP, terrain, etc.)

Comment est organisée

l'interdisciplinarité

dans les enseignements ?

Nombre moyen d'étudiants

(par année)

Origines disciplinaires

des étudiants

Comment est prise en charge l'hétérogénéité des étudiants?

Existe-t-il des échanges entre étudiants à l'occasion de travaux collectifs?

Débouchés envisagés et/ou effectifs

\section{Nombre d'enseignants}

Master issu d'un DESS créé en 1998 (GPE : Gestion et protection de l'environnement)

Université de Lille 3

Universités de Lille 1, Lille 2 et l'Université du littoral côte d'opale (Ulco)

Réseau « Développement durable et Territoires fragiles »

Clerse-Ifresi, axe « Territoire, Environnement, Développement

Durable »

Économie, gestion, droit

Formation à « double compétence » : une dans une discipline de sciences sociales ou de sciences de la vie ou de la Terre et une en économie et en gestion. Il s'agit de combler un vide du côté des sciences sociales dans les formations aux sciences de l'environnement.

-Cours,

-Stage en entreprise dans chacune des deux années, visites de terrain

-Projets par équipes mixtes

- Travail en groupe avec rendu

Il s'agit plus d'une double formation que d'une formation interdisciplinaire. Celle-ci se fait à travers des initiations aux disciplines complémentaires à celles des formations initiales des étudiants, ainsi qu'à travers des « modules interdisciplinaires et interuniversitaires», des projets par équipes mixtes et un travail en groupe avec rendu.

M $1: 15$ à 20

M $2: 20$ à 25

Les étudiants proviennent d'un cursus qui est soit de sciences sociales, soit de sciences de la vie ou de la Terre.

Une partie importante accède directement en M 2.

Les étudiants suivent des cours d'initiation aux disciplines qu'ils n'ont pas étudiées auparavant.

Ils participent à des projets transversaux qui permettent une mise en commun des connaissances (disciplines de sciences appliquées d'une part, disciplines de sciences humaines et sociales, de l'autre).

Les échanges s'effectuent avec les étudiants de la formation et avec les étudiants de formations d'autres universités (scientifiques) et intéressées à développer une politique de site.

Il s'agit de donner à ces étudiants une compétence leur permettant d'occuper des fonctions en rapport avec la protection de l'environnement dans les organisations territoriales ou dans les entreprises.

Le secteur d'embauche reste en fait très lié à la formation initiale (ex :les biologistes dans les PNR, les géographes et les économistes dans les collectivités, les physiciens et les ingénieurs dans les entreprises ISO).

Une vingtaine 


\section{Statuts des enseignants (universitaire, chercheur, ingénieur...) \\ Y a-t-il concertation entre enseignants? \\ Sous quelle forme?}

\section{Difficultés rencontrées}

Propositions, pistes pour des améliorations
Universitaires, chercheurs (conférences)

Professionnels (entreprises, collectivités locales)

- Réunions pédagogiques, échanges par messagerie électronique, discussions informelles lors des jurys.

-Fiches d'évaluation des étudiants en stage demandées aux étudiants et au tuteur présent à la soutenance.

- La mise à niveau des étudiants dans les disciplines qui sont nouvelles pour eux pose des problèmes d'ajustements pédagogiques. Il s'agit en effet de croiser des cultures scientifiques de sciences de la vie ou de la Terre et de sciences sociales.

- Il faut aussi ajuster l'enseignement aux attentes des offreurs de débouchés.

- Le contenu technique des formations est parfois insuffisant au regard d'une spécialisation disciplinaire de type économie-gestion.

- Approfondir les coopérations avec les universités scientifiques de manière à mieux produire le melting pot attendu.

- Faire connaître les recherches de type pluridisciplinaires associées au master.

- Développer le suivi post universitaire de manière à avoir plus de retours sur la formation.

\section{Archéologie et histoire (Dominique MARGUERIE)}

Intitulé de la formation en 2007

Intitulé de la (des)
spécialité(s) ou option(s)

Niveau (master 1, 2)

Master Pro ou recherche

Responsable(s)

Date de création

Institutions de rattachement

Institutions associées
Archéologie et histoire

Master 2 recherche :

1. Archéologie et archéosciences : Préhistoire, Protohistoire

2. Archéologie et histoire des mondes antiques

3. Archéologie et histoire des mondes médiévaux et modernes

Master 2 Pro

4. Métiers de l'archéologie

Master 1 (Rennes 2)

Master 2 (Rennes 1, Rennes 2, Nantes)

Master 2 recherche : spécialité 1 (Rennes 1), spécialités 2 et 3

(Rennes 2)

Master Pro : spécialité 4 (Nantes)

Annie ANTOINE (Professeur d'histoire moderne, université Rennes 2, annie.antoine@uhb.fr)

Septembre 2004

Originalité : association de trois universités :

- Rennes 2 (Master 1et Master 2 recherche)

- Rennes 1 (Master 2 recherche)

- Nantes (Master Pro)

CNRS, ministère de la Culture, Inrap 


\section{Laboratoire(s) d'accueil}

\section{Disciplines enseignées}

\author{
Objectif(s) de \\ l'enseignement \\ (compétences \\ interdisciplinaires \\ recherchées)
}

Descriptif de l'enseignement (cours, TP, terrain, etc.)

\section{Comment est organisée l'interdisciplinarité dans les enseignements ?}

Nombre moyen d'étudiants (par année)

Origines disciplinaires des étudiants

Comment est prise en charge l'hétérogénéité des étudiants ?

Existe-t-il des échanges entre étudiants à l'occasion de travaux collectifs?

\section{Débouchés envisagés et/ou effectifs}

Nombre des enseignants

Statuts des enseignants (universitaire, chercheur, ingénieur, etc)

Y a-t-il concertation entre enseignants? Sous quelle forme?
UMR 6566 CReAAH (ses laboratoires de Rennes 1, Rennes 2 et Nantes et son musée de la Préhistoire de Penmarch)

-Archéologie de la préhistoire, de la protohistoire, des périodes antiques et médiévales.

- Sciences de la vie et de la matière appliquées à l'archéologie : paléoécologie, palynologie, anthracologie, dendrologie, chimie organique et minérale, pétrographie, céramologie, paléométallurgie, malacologie, sédimentologie.

Former des archéologues dotés d'une culture archéologique approfondie et d'une bonne expérience de terrain et de laboratoire.

L'interdisciplinarité recherchée est celle de l'archéologie. Mais s'ajoute le souci de faire le lien entre science archéologique et histoire. Une partie des enseignements se situe au point de rencontre de ces disciplines.

\section{Deux semestres :}

- le premier axé sur une formation théorique dense qui porte autant sur la culture archéologique générale que sur la connaissance des disciplines archéométriques et des techniques et méthodes de l'archéologie ;

- le second consacré à des stages de laboratoire et de fouille.

Par les spécialités de recherche des enseignants (chercheurs ou enseignant-chercheurs) et par les stages de laboratoire.

\section{0 en M 1}

14 en M 2

Histoire, Histoire de l'Art et Archéologie, Géologie, Physique, Chimie, Biologie...

- Adaptation des enseignements ;

- aide bibliographique ;

- cours de mise à niveau.

Oui

Tous les métiers exigeant une compétence en archéologie programmée ou préventive (recherche, muséographie, éducation, administration...)

Une vingtaine en master 2

30 en M 2-1

- Chercheurs CNRS

-Enseignant-Chercheurs des universités de Rennes 1, Rennes 2

et Nantes

- Ingénieurs du CNRS, ministère de la Culture

- Ingénieurs-chargés de recherche de l'Inrap

Oui, sous la coordination des responsables des enseignements. 


\section{Difficultés rencontrées}

Propositions, pistes pour des améliorations

Source
- Le principal problème est l'insuffisance notoire des heures de «présentiel »-Étudiants à Rennes 2 (le M I est cadré par Rennes 2) : la formation des archéologues nécessiterait, en M 1, un nombre important d'heures de travaux dirigés et de travaux pratiques, en plus des heures de cours magistraux (apprentissage du dessin archéologique, de la techno-typologie céramique et lithique, etc.); - Une difficulté corrélative est le trop faible nombre d'enseignantschercheurs à l'université de Rennes 1 (1 seul MCF) ;

- Parallèlement, maintenir un mémoire en M I n'est pas indispensable (sa place est en M II Recherche, si possible sous la forme d'un article scientifique à l'issue du stage en laboratoire) - http://www.uhb.fr/

- Augmenter le « présentiel » étudiants

- Créer des TP et TD adaptés

- Supprimer le mémoire en M I : Le master I n'est plus la « maîtrise »!

http://www.uhb.fr/jsp/fiche pagelibre.jsp?

STNAV $=904 \&$ RUBNAV $=\&$ CODE $=36462303 \&$ LANGUE $=0$

\section{Didactique des sciences et techniques (Jean-Louis MARTINAND)}

Intitulé de la formation en 2006

\section{Domaine}

Intitulé de la (des)

spécialité(s) ou option(s)

Niveau (master 1, 2)

Master Pro ou recherche

\section{Responsable(s)}

Date de création

Historique

Institutions de rattachement Institutions associées
Didactique des sciences et techniques

\section{Sciences et technologie}

Cette formation à la didactique des sciences et techniques est le parcours recherche du master : «Communication scientifique et technique : contenus, outils, pratiques » de l'ENS Cachan.

Master 2 : recrutement sur master 1 scientifique ou technologique

Master 2 recherche (il existe par ailleurs un master II pro dans le master « Communication scientifique et technique : contenus, outils, pratiques »)

Jean-Louis MARTINAND, Joël LEBEAUME, professeurs, UMR STEF, École normale supérieure de Cachan

2004

Suite d'un DEA de didactique des sciences et techniques créé à l'ENS Cachan en 1992 ; DEA issu d'un DEA de Paris 7 créé en 1974.

École normale supérieure de Cachan

Deux séminaires pris dans deux universités peuvent faire partie des options du master : le séminaire «Recherches historiques sur l'enseignement et la diffusion des sciences et des techniques » (Paris Sud Orsay) et le séminaire du « Groupe d'Étude didactique et d'intervention dans le domaine des activités physiques et sportives » (Paris 12).

Le Palais de la Découverte fournit un séminaire, un enseignant et une structure d'accueil pour les étudiants. 
Laboratoire(s) d'accueil

\section{Disciplines enseignées (cours)}

\author{
Objectif(s) de \\ l'enseignement \\ (compétences \\ interdisciplinaires \\ recherchées)
}

Descriptif de l'enseignement
(cours, TP, terrain, etc.)

Comment est organisée

l'interdisciplinarité

dans les enseignements ?

Nombre moyen d'étudiants

(par années)

Origines disciplinaires

des étudiants

Comment est prise en charge l'hétérogénéité des étudiants?

\author{
Existe-t-il des échanges \\ entre étudiants à l'occasion \\ de travaux collectifs?
}

- UMR Sciences Techniques Éducation Formation (STEF) de l'ENS de Cachan et de l'INRP

- Unité Études sur les sciences et les techniques (Paris Sud Orsay)

- Groupe d'étude didactique et intervention dans le domaine des Activités physiques et sportives (Paris 12)

- Laboratoire de pédagogie des sciences (Université de Louvain, Belgique)

- Laboratoire de didactique et d'épistémologie des sciences

(Université de Genève, Suisse)

- Palais de la Découverte

- Méthodologie et techniques de la recherche en éducation ;

- Epistémologie et concepts pour la didactique des sciences et techniques ;

- Tendances des recherches en :

- didactique des sciences expérimentales et des disciplines techniques

- technologie de l'information pour l'éducation

- médiatique des sciences et techniques

- histoire de l'enseignement et de la diffusion des sciences.

Préparer aux recherches sur l'enseignement, l'apprentissage et la diffusion des sciences et des techniques, sur leur histoire, sur la technologie de l'éducation et de la diffusion. Le point de vue privilégié est celui des contenus scientifiques enseignés ou diffusés ou des techniques d'information et de communication utilisées. Ces recherches visent aussi bien la connaissance que l'intervention.

L'enseignement comporte des enseignements généraux et quatre options :

- didactique des sciences expérimentales et des disciplines

technologiques ;

- technologie de l'information et de la communication pour

l'enseignement et la diffusion;

- médiatique des sciences ;

- histoire de l'enseignement et de la diffusion des sciences.

Il donne une place importante aux travaux personnels.

Les enseignements sont par nature transversaux par rapport aux disciplines « objets » (sciences, technologie) et disciplines « outils » (sciences humaines et sociales, ingénierie culturelle).

Une vingtaine

Sciences et génies techniques

Par le caractère transversal du contenu de l'enseignement qui s'appuie sur la culture scientifique acquise, quelle qu'elle soit, pour l'aborder sous l'angle de la didactique. Par ailleurs, une place importante est donnée aux travaux personnels (stage de recherche, note bibliographique, séminaires spécialisés).

Pas de travaux spécifiquement collectifs, mais séances collectives de discussions pour les mémoires. 


\section{Débouchés envisagés et/ou effectifs}

Nombre des enseignants

Statuts des enseignants (universitaire, chercheur, ingénieur, etc.)

$Y$ a-t-il concertation entre enseignants?

Sous quelle forme?

Difficultés rencontrées

Propositions, pistes pour des améliorations
La recherche en didactique des sciences et des techniques, technologie de l'éducation, la formation des enseignants et des médiateurs.

Sept; participation des chercheurs à l'encadrement individuel.

Universitaires (ENS de Cachan, IUFM, INRP, universités, Palais de la Découverte)

Construction des enseignements en fonction des compétences et responsabilités des enseignants ; jurys collectifs pour les soutenances.

- Passage de la posture d'enseignant et surtout de formateur qui jugent la conformité de réponses, à la posture de chercheur qui veut comprendre une pensée différente, ou concevoir des contenus ou dispositifs nouveaux.

- Absence d'habitude de rédaction développée et de lecture analytique, manque d'exigence de rigueur conceptuelle concernant l'éducation, à la fois pratique familière et objet d'étude scientifique.

Travaux d'analyse de textes; proposition d'ateliers d'écriture.

\section{4. Écologie humaine : enjeux environnementaux des activités de production et de consommation (Francis RIBEYRE)}

Intitulé de la

formation(titre)

Intitulé de la (des)

spécialité(s) ou option(s)

Niveau (master 1, 2)

Master Pro ou recherche

Responsable(s)

Date de création

Institution(s)

de rattachement

Institutions associées
Écologie humaine : enjeux environnementaux des activités de production et de consommation

Mention : Géographie et Aménagement (UFR Géographie et Aménagement)

Spécialité : Territoires, Développement et cultures, Gestion environnementale

Master 2

Master Pro

Francis RIBEYRE, Professeur

E-mail : ribeyre@egid.u-bordeaux.fr

Institut Environnment, géo-ingénierie et développement (Egid)

Université Bordeaux 3

1 allée Daguin

33607 Pessac Cedex

Tél. : 0557121033

2007

Institut Egid et université Bordeaux 3

Université Michel de Montaigne Bordeaux 3 


\section{Disciplines enseignées}

\author{
Objectif(s) de \\ l'enseignement \\ (compétences \\ interdisciplinaires \\ recherchées)
}

\author{
Descriptif de \\ l'enseignement \\ (cours, TP, terrain, etc.)
}

Écologie entrepreneuriale (systèmes de management environnementaux, écoconception, écotourisme...), écologie urbaine (écosites, Agendas $21 \ldots$ ), écologie familiale (écoachats, écocitoyenneté, écohabitat...).

1. Objectifs scientifiques : il s'agit de former les étudiants à prévoir, diagnostiquer et gérer les incidences des nouveaux modes de vie et des évolutions socio démographiques sur les écosystèmes, notamment au travers des modifications de la consommation de biens et services par les citoyens : habitat, transport, alimentation, équipements domestiques, loisirs, services publics... Il s'agit également de fournir aux étudiants des stratégies d'analyse et d'intervention applicables au fonctionnement des organisations, à l'évaluation des risques, à la gestion de crise permettant de renforcer la cohérence des diverses actions sectorielles conduites (gestion des déchets, économies d'énergie, qualité environnementale des logements ...) et d'optimiser les coûts financiers.

2. Objectifs professionnels : le but est de former des spécialistes capables d'appréhender la complexité des problématiques environnementales, de comprendre les liens entre écologie et acteurs du territoire, et ainsi de positionner les enjeux écologiques en relation avec les attentes sociales, dans le cadre de programmes de développement local et de stratégies d'entreprises.

- UE 1 (96 h) : tronc commun partagé par les trois parcours de la spécialité :

- 1 séminaire à choisir parmi 3 : Nouveaux enjeux géographiques et politiques de développement au Sud ; Lieux, territoires, terroirs, épistémologie des structures socio-spatiales ; Environnement, biens publics et développement durable ;

-5 cours à choisir parmi 10 : Systèmes productifs spatialisés et développement durable ; Systèmes d'acteurs, gouvernance et échelles de l'action; Formes, dynamiques et aménagement des territoires urbains ; Composantes culturelles et sociales et politiques des systèmes environnementaux ; Modes de gestion des biens publics environnementaux ; Fonctionnement des systèmes environnementaux; Outils et méthodes d'analyse (représentations de l'espace 1 et 2 ; enquêtes ; écosystèmes.

- UE 2 (288 h, dont $120 \mathrm{~h}$ de projet tutoré) : spécifique au parcours d'Écologie humaine :

- 5 modules obligatoires (Impacts écologiques des activités humaines 1 - production et distribution; Impacts écologiques des activités humaines 2 - consommation des ménages et rejets ; Méthodes intégrées de diagnostic environnemental; Formation à la communication et gestion de crise ; Éducation à l'environnement) ; - 1 Module d'échange à choisir parmi 5 (Approche interdisciplinaire des enjeux écologiques ; Gestion économique de l'environnement ; Histoire et usages des Sciences ; Maîtrise des risques naturels et techniques ; Gestion globale de la ressource en eau );

- Projets tutorés et gestion de projet.

- UE 3 : stage de 6 mois en situation professionnelle (entreprise, collectivité locale...). 


\author{
Comment est organisée \\ l'interdisciplinarité \\ dans les enseignements ?
}

Nombre moyen d'étudiants (par années)

\author{
Origines disciplinaires \\ des étudiants
}
Existe-t-il des échanges entre étudiants à l'occasion de travaux collectifs?

\section{Nombre d'enseignants}

Statuts des enseignants (universitaire, chercheur, ingénieur, etc.)
Acquisition de connaissances pluridisciplinaires et approfondies dans les domaines des sciences de la vie, de la terre et des sciences humaines et, surtout, nécessité de les interconnecter. L'apport de ces connaissances est orienté vers la compréhension des relations existant entre les activités humaines et l'environnement, en s'appuyant sur diverses facettes de l'écologie.

Au cours de ce parcours, l'intervention de professionnels, les nombreuses études de cas, la réalisation de projets, ainsi qu'un stage de six mois dans un organisme, prépareront les étudiants au monde professionnel.

\section{0}

Étudiants titulaires d'un M 1 de sciences biologiques, sciences de la terre, sciences humaines et sociales, sciences économiques, sciences politiques

Dans le cadre de projets tutorés

L'objectif de ce parcours professionnalisant est de préparer les étudiants aux métiers sectoriels, mais aussi et surtout transversaux, de l'environnement, dans un contexte où l'incidence des activités humaines sur l'environnement est de plus en plus prise en compte dans des secteurs aussi variés que la production, la distribution, la consommation ou encore la gestion territoriale.

Cette formation doit donc permettre aux futurs diplômés de mettre en œuvre les politiques de management environnemental d'écogestion et d'écoconseil dans les collectivités territoriales, les entreprises, les organismes de gestion, les associations... Il s'agit également de former des acteurs de la médiation environnementale et de l'éducation à l'environnement et à la consommation durable en réponse à une demande de formation des personnels des entreprises et des collectivités.

Sont visés les métiers nécessitant des compétences permettant d'aborder les problèmes environnementaux dans leur globalité et non pas seulement de façon sectorielle ou purement technique. Les structures susceptibles de recruter les étudiants de ce parcours relèvent de domaines d'activité très variés tels que les secteurs de la production, de la distribution, des études et projets, de la consommation durable, de la gestion publique, de la formation et des services : entreprises industrielles ; secteur tertiaire ; bureaux d'études ; PME et PMI ; grande distribution (alimentaire, bricolage, jardinage ...) ; collectivités territoriales ; secteur associatif.

Une trentaine d'intervenants

Universitaires, professionnels

Enseignants : outre les chercheurs et enseignants il est fait appel à des professionnels extérieurs sensibilisés à l'écologie humaine, volontaires souhaitant relever le défi, personnes intéressées par cette formation, personnes capables de vulgariser. 
Difficultés rencontrées

Propositions, pistes pour des améliorations

Sources
Expérience trop récente

En relation avec les activités de recherche et d'enseignement en écologie humaine dispensés à l'Institut Egid et dans diverses formations depuis plusieurs années, un réseau de collaborations est en place avec les acteurs du territoire dans différents domaines d'activité : entreprises privées, bureaux d'études d'ingénierie, administrations et services des collectivités qui exercent une activité dans les domaines de la gestion de l'environnement, des géoressources, de l'eau, de la géoimagerie, des risques, des banques.

http://www.egid.u-bordeaux.fr/presentation.htm http://www.egid.u-bordeaux.fr/formation/iframes/masterecohumaine.htm

\section{Certificat international d'écologie humaine (CIEH) (Francis RIBEYRE)}

Intitulé de la formation
(titre)

Niveau (master 1, 2)

Responsable(s)

Date de création

Institution(s)

de rattachement

Institutions associées

Disciplines enseignées

Objectif(s) de

l'enseignement

(compétences

interdisciplinaires

recherchées)

Descriptif de

l'enseignement (cours, TP, terrain, etc.)

Comment est organisée

l'interdisciplinarité

dans les enseignements ?

Nombre moyen d'étudiants (par années)
Certificat international d'écologie humaine (CIEH)

Master 1 et 2

Francis RIBEYRE, Professeur

E-mail : ribeyre@egid.u-bordeaux.fr

Institut Egid, Université Bordeaux 3

1 allée Daguin

33607 Pessac Cedex

Tél. : 0557121033

1974

Université Bordeaux 1

Universités de Bordeaux 2, Bordeaux 3 et Bordeaux 4 et neuf universités européennes

Écologie humaine, environnement, gestion des risques, santé, communication, psycho-sociologie, économie...

Le certificat international d'Écologie humaine au travers des nombreux thèmes abordés, contribue à élargir le champ des connaissances acquises antérieurement et incite à une réflexion interdisciplinaire sur les interactions entre l'Homme et les écosystèmes.

- 100 heures de séminaire en première année

- Travail de recherche en seconde année

Séminaires pluridisciplinaires complétés par des synthèses

30-40 issus de différentes formations initiales. Une dizaine d'étudiants sont en activité professionnelle dans divers domaines (enseignement, santé...) 
Origines disciplinaires des étudiants

Existe-t-il des échanges entre étudiants à l'occasion de travaux collectifs ?

Débouchés envisagés et/ou effectifs

Nombre d'enseignants

Statuts des enseignants (universitaire, chercheur, ingénieur, etc.)

Y a-t-il concertation entre enseignants?

Sous quelle forme?

Difficultés rencontrées

Source
Minimum requis : master 1 en formation initiale VAE et VAP pur les personnes en activité professionnelle

En seconde année : présentation mensuelle de l'état d'avancement des travaux de recherche

Pas de ciblage professionnel, mais complément à une formation initiale (médecine, architecture, droit, écologie...)

Une quarantaine d'intervenants

Universitaires, professionnels

Entre les responsables pédagogiques

Système fonctionnant depuis plus de trente ans

http://www.dfc.u-bordeaux1.fr

\section{Environnement : milieux, techniques, sociétés (EMTS) (Marie ROUE)}

Intitulé de la formation(titre)

Intitulé de la (des) spécialité(s) ou option(s)

\section{Niveau (master 1, 2)}

Master Pro ou recherche

Responsable(s)

Date de création

Historique

Institution de rattachement

Institutions associées
Master « Évolution, patrimoine naturel et sociétés » du Museum national histoire naturel (MNHN)

Spécialité « Environnement : milieux, techniques, sociétés « (EMTS) Parcours recherche :

- Ethnoécologie, savoirs locaux et gestion de la biodiversité (MNHN);

- Paysages, milieux et développement durable (Paris 7) ;

- Développement durable et agriculture (AgroParisTech).

M 1 et M 2, mais seulement M 2 cohabilité

Master recherche

- Pour le MNHN : Serge BAHUCHET, Professeur ; Marie ROUÉ, DR CNRS

- Pour Paris 7 : Marianne COHEN, MC

- Pour AgroParisTech : Jean LOSSOUARN, Professeur

1995

DEA Environnement : temps, espaces, sociétés (ETES) (1995-2000) DEA EMTS (2000-2004)

Master EMTS (2004-8)

Muséum national d'histoire naturelle

AgroParisTech et université Paris 7 Denis Diderot pour le M 2 
Laboratoire(s) d'accueil

\section{Disciplines enseignées}

Objectif(s) de l'enseignement (compétences interdisciplinaires recherchées)

\section{Descriptif de l'enseignement (cours, TP, terrain, etc.)}

Comment est organisée l'interdisciplinarité dans les enseignements?

Nombre moyen d'étudiants (par années)
Principaux :

-UMR 5145 « Eco-anthropologie et Ethnobiologie » pour MNHN; -Ladyss (laboratoire dynamiques sociales et recomposition des espaces) pour Paris 7 ;

-Sadapt (Sciences, action, développement, activités, produits, territoires) pour AgroParisTech.

Sciences humaines et sociales, sciences agronomiques et sciences écologiques

Approche interdisciplinaire qui associe sciences sociales et humaines, sciences agronomiques et sciences écologiques afin d'éclairer les relations entre sociétés et nature. Mieux comprendre les relations entre processus écologiques et activités humaines permet d'agir sur elles avec davantage de pertinence et d'efficacité. La préservation de l'environnement passe par le développement de recherches interdisciplinaires.

Au moment où l'on passe de la protection de l'environnement qui était du ressort des seuls décideurs et scientifiques à une problématique de co-gestion, les points de vue des acteurs sociaux se confrontent. Aucune discipline ne dispose à elle seule des outils nécessaires pour comprendre cette complexité.

L'année s'organise en trois temps : tronc commun, parcours au choix, stage de recherche :

- le tronc commun approfondit et croise les regards portés par ces sciences sur l'environnement. Il propose de dépasser les clivages disciplinaires et cherche à favoriser le dialogue entre étudiants de cursus différents et chercheurs de domaines variés ;

- les trois parcours déclinent l'interdisciplinarité en environnement selon des angles de vue distincts :

1.Ethnoécologie, savoirs locaux et gestion de la biodiversité (ESLGB),

2.Développement durable et agricultures (DDA),

3.Paysages, milieux et développement durable (PMDD) ; - stage donnant lieu à un mémoire.

- Ouverture des étudiants de sciences sociales aux questions environnementales et des étudiants de sciences écologiques aux questions de société pour réaliser des programmes de recherche et de développement pluri- et interdisciplinaires.

- Capacité à travailler en équipe et à sortir de son champ disciplinaire.

- Sensibilisation aux problèmes d'éthique dans les relations avec les sociétés locales.

- Acquisition de méthodes d'enquêtes et d'analyse de données de plusieurs disciplines

- Capacité de synthèse de données d'origine variée (résultats d'enquêtes, littérature scientifique et de vulgarisation, données iconographiques et sonores, données écologiques et géographiques, etc.) et de rédaction de rapports scientifiques. 


\section{Origines disciplinaires des étudiants}

Comment est prise en charge l'hétérogénéité des étudiants?

Existe-t-il des échanges entre étudiants à l'occasion de travaux collectifs?

Débouchés envisagés et/ou effectifs

Nombre d'enseignants

Statuts des enseignants (universitaire, chercheur, ingénieur, etc.)

$\mathrm{Y}$ a-t-il concertation entre enseignants? Sous quelle forme?

Difficultés rencontrées

\section{Résultats}

Source
Agronomie, anthropologie, biologie,droit, écologie, ethnologie, géographie, sciences de l'environnement, sciences de la santé, sociologie, etc.

Tutorat, mais aussi prise en compte par le groupe d'étudiants lui même qui passe en dehors des cours beaucoup de temps à discuter et confronter les points de vue.

Oui. Organisation de «mini-colloques » ou de jeux de rôle où les points de vue des disciplines se complètent et se confrontent. Egalement rédaction de rapports communs sur la base des différentes origines disciplinaires.

- Préparation d'un doctorat (3 ans) nécessaire pour être candidat à des postes de chercheurs et d'enseignants-chercheurs. Le doctorat peut se dérouler à l'École doctorale du Muséum « Sciences de la nature et de l'homme » ou dans une autre École doctorale (universités, institutions de recherche et d'enseignement).

- Emplois dans les services d'aménagement et d'environnement des collectivités territoriales (département, région, etc.), dans les établissements publics ou privés, les entreprises et les bureaux d'études chargés de l'aménagement du territoire et de l'environnement, de l'agriculture, de l'énergie, de l'équipement et des transports.

- Emplois dans des Organisations non gouvernementales (ONG), des Institutions non gouvernementales (ING), des associations œuvrant dans les domaines de l'environnement, du développement, de la reconnaissance des sociétés locales, de la protection de la nature, de la santé, etc.

- Chargés de mission au sein de structures de gestion d'espaces protégés (Parcs nationaux, Parcs naturels régionaux, Réserves naturelles, etc.), d'organismes chargés de la gestion forestière (ONF, IDF), du gibier et de la faune sauvage (ONCFS), etc.

Environ $60+$ intervenants ponctuels

Enseignants-chercheurs et chercheurs du Muséum et du CNRS, Professeurs d'AgroParisTech et de Paris 7 Denis Diderot

Réunions pédagogiques et cours à 2 ou 3 voix

Temps d'adaptation nécessaire pour passer de la discipline d'origine à une bonne pratique de l'interdisciplinarité

Satisfaction des étudiants en fin de M 2 ; au MNHN, selon les années : entre $1 / 3$ et $1 / 2$ de la promotion en thèse, $1 / 3$ d'emplois dans le secteur environnement, $1 / 3$ de poursuite d'études (autres masters, agrégations...)

www.mnhn.fr/master et Marie Roué 


\section{Modèles et systèmes en biologie : approches interdisciplinaires du vivant (AIV) (François TADDEİ, Livio RIBOLI-SASCO)}

\author{
Intitulé de la formation \\ en 2007 \\ Intitulé de la (des) \\ spécialité(s) ou option(s)
}

Niveau (master 1 et 2)

Master Pro ou recherche

Responsable(s)

Date de création

Historique

Institutions de rattachement

Institutions associées

Laboratoire(s) d'accueil

\section{Disciplines enseignées}

\author{
Objectif(s) de \\ l'enseignement \\ (compétences \\ interdisciplinaires \\ recherchées)
}

\author{
Descriptif \\ de l'enseignement \\ (cours, TP, terrain, etc.)
}

Sciences de la vie et de la santé

Mention : Modèles et systèmes en biologie : approches interdisciplinaires du vivant (AIV)

Master 2

Master recherche et pro

François TADDEÏ, Inserm, faculté de médecine de Paris Descartes CRI (Centre de recherches interdisciplinaires)

Université Paris Descartes - Site Cochin Port-Royal

24, rue du Faubourg St Jacques, 75014 - Paris

Tél. : 0144412222

2004

Création de chercheurs et d'étudiants motivés

Universités de Paris VII - Diderot

École normale supérieure et Paris V

Les laboratoires peuvent appartenir à toute université ou institution de Paris et de ses environs de toutes les disciplines prises en compte dans le master.

Les Sciences du Vivant, en s'appuyant sur les différentes disciplines des étudiants (Mathématiques, Informatique, Biologie, Médecine, Chimie, Physique, Science de l'ingénieur, Sciences cognitives).

Former des chercheurs susceptibles d'ouvrir la biologie à d'autres disciplines. L'accent est mis sur le développement de l'esprit critique, des capacités d'analyse et de synthèse. Le master veut contribuer à une réflexion intégrée sur l'interdisciplinarité et son apport pédagogique. Il se définit plutôt par une méthode pédagogique et par le domaine auquel cette méthode est appliquée : les sciences du vivant.

- Stages : Les étudiants d'AIV effectuent trois stages d'un trimestre chacun. Les laboratoires peuvent appartenir à toute université ou institution de Paris et de ses environs. La seule obligation est de réaliser un stage théorique et un stage expérimental. Les étudiants sont encouragés à contacter et visiter autant de laboratoires que nécessaire afin de choisir un projet et un laboratoire qui correspondent à leur intérêt personnel. A la fin de chaque stage, les étudiants présentent leur travail sous la forme d'un séminaire de 30 minutes.. C'est au cours de ces stages qu'a lieu l'ouverture à de nouvelles pratiques méthodologiques, mais aussi à des façons d'envisager le travail scientifique propres à chaque discipline, et parfois propres à chaque laboratoire ou institut de recherche.

- Atelier : Regroupement des étudiants et des chercheurs provenant de disciplines différentes pour concevoir ensemble des projets créatifs à l'interface avec les sciences de la vie. Les participants apprennent à travailler et à réfléchir ensemble à travers différents 


\section{Comment est organisée l'interdisciplinarité dans les enseignements ?}

ateliers et séances de brainstorming sur les questions ouvertes des sciences de la vie. À cette exercice s'ajoute l'évaluation des besoins financiers. Cet apprentissage, absent de la plupart des formations, est essentiel dans le contexte actuel de la recherche où l'obtention de financements est la base de toute réalisation d'étude. En fin d'année, les étudiants sont également encadrés afin d'établir leur projet de thèse.

- Cours : L'interdisciplinarité se pratique aussi à travers les exercices bibliographiques réalisés en binôme pluridisciplinaire et exposés à l'ensemble des étudiants. Ces exposés sont à chaque fois suivis de longues discussions et de propositions de travaux expérimentaux qui pourraient être mis en place afin de répondre à certains des questionnements suscités par ces échanges.

$-1^{\text {er }}$ trimestre : Analyse critique d'un article scientifique ${ }^{28}$ Une série de séminaires est présentée par des binômes d'étudiants, chacun des membres étant issu d'une formation distincte. Chacun des binômes présente en détail un article scientifique de son choix : énoncé des hypothèses sous-jacentes, description des techniques utilisées et discussion des résultats. Suite à leur analyse, les étudiants proposent des approches expérimentales ou des modélisations complémentaires.

$-2^{\mathrm{e}}$ trimestre : Synthèse bibliographique. Une série de séminaire, chacun préparé par deux étudiants de formations différentes présentant leur point de vue sur un sujet scientifique de leur choix à travers l'exploration de la littérature spécialisée. Une présentation concise du résultat de cette exploration, du point de vue de leur discipline initiale est attendue de la part des étudiants, suivie d'une discussion autour des questions ouvertes du thème choisi ainsi que la proposition d'un projet de recherche. $-3^{\mathrm{e}}$ trimester : 'Zoom out-Zoom in ${ }^{29}$. Avec un ouvrage scientifique comme point de départ, les étudiants (en binôme) présentent la thèse de l'ouvrage et proposent un projet de recherche cohérent sous la forme d'une demande de financement (e.g. ANR) pour un travail de thèse. Pour chaque dossier de demande, un binôme sera désigné comme rapporteur.

- Clubs : Les étudiants mettent en place en cours d'année des petits groupes de discussion thématique sur les sujets qui les intéressent le plus. Avec l'aide des encadrants, ils se réunissent autour de lectures de publications récentes ou en invitant des chercheurs du domaine. Ces groupes offrent notamment un appui critique aux étudiants dans l'élaboration de leur projet de thèse.

Il ne s'agit pas à proprement parler d'enseignements, mais d'un ensemble d'activités qui ont comme objectif de faire l'apprentissage d'une conception et d'une mise en œuvre de l'interdisciplinarité autour du vivant.

Au sein du master AIV, l'interdisciplinarité est déclinée sous trois formes :

- un enrichissement méthodologique par la pratique en laboratoire de différentes disciplines ;

- un questionnement à la frontière entre disciplines ;

- des regards croisés sur les pratiques scientifiques au quotidien.

\footnotetext{
${ }^{28} \mathrm{http}: / /$ www.lille.webstore.fr/cri/index.php?option=com_content\&task=blogcategory\&id=83\&Itemid=105

${ }^{29} \mathrm{http}$ ://www.lille.webstore.fr/cri/index.php?option=com_content\&task=blogcategory\&id=85\&Itemid=107
} 


\author{
Nombre moyen d'étudiants \\ (par années)
}

\author{
Origines disciplinaires \\ des étudiants
}

\section{Comment est prise en charge l'hétérogénéité des étudiants?}

\section{Existe-t-il des échanges entre étudiants à l'occasion de travaux collectifs?}

\section{Débouchés envisagés et/ou effectifs}

\section{Nombre d'enseignants \\ Statuts des enseignants (universitaire, chercheur, ingénieur...)}

$Y$ a-t-il concertation entre enseignants? Sous quelle forme?

\section{Difficultés rencontrées}

\section{Propositions, pistes pour des améliorations}

Une quinzaine

Étudiants des Grandes Écoles et des universités françaises et étrangères ayant suivi un cursus approfondi dans les disciplines suivantes : biologie, médecine, physique, chimie, mathématiques, informatique, science de l'ingénieur.

À travers la multiplicité et la souplesse des modalités du cursus impliquant toutes les disciplines couvertes par le master.

Cette pédagogie interdisciplinaire offre aux étudiants :

-une plus grande mobilité dans la pratique de recherche ;

- une souplesse de questionnement et de formulation d'hypothèses innovantes face aux découvertes expérimentales ;

-une plus grande maturité critique.

Les étudiants prenant en charge eux-mêmes la conception et la réalisation de leur formation, la coopération sur des objectifs communs d'étudiants de disciplines différentes induit un processus de formation mutuelle à travers les modalités de formation choisies d'un commun accord. De plus pour chaque exercice décrit ci-dessus les étudiants : travaillent en binômes interdisciplinaires.

Taux de réussite ( 3 ans): $95 \%$. Insertion professionnelle: $80 \%$ thèse; $8 \%$ thèse étranger; $3 \%$ chercheur secteur prive ; $6 \% \mathrm{MD} ; 3 \%$ chercheur titulaire (CNRS) Débouchés visés : chercheurs dans les organismes de recherche ou privé.

\section{4}

Universitaires (ENS, universités), chercheurs

Réunion hebdomadaire à l'issue des cours

Le master est nouveau. Il découvre les difficultés de l'apprentissage de l'interdisciplinarité. Il exige un travail beaucoup plus intense que pour un master 2 classique. Il exige un niveau d'excellence des étudiants. Son caractère interdisciplinaire a parfois créé des difficultés administratives pour le passage en thèse. Le suivi de la formation en thèse s'est parfois confronté au découpage disciplinaire des écoles doctorales. La mise en place d'une ED interdisciplinaire européenne Frontières du Vivant a permis de dépassé ces difficultés.

Le dynamisme de ce groupe d'étudiants et de chercheurs, sa richesse méthodologique due à la diversité des cursus des étudiants, sa capacité à poser des questions originales et à offrir une critique puissante et constructive du fait des regards croisés de disciplines variées, nous a incités à établir des structures permettant de maintenir cette interaction scientifique fructueuse.

Nous avons donc ouvert une structure accueillant les étudiants et chercheurs désireux de travailler dans cet état d'esprit : le CRI ${ }^{30}$.

\footnotetext{
${ }^{30}$ Situé au cœur de Paris à 5 minutes du jardin du Luxembourg, au sein de la faculté de Médecine Paris Descartes sur le site de Cochin, le CRI (Centre de recherches interdisciplinaires) a été fondé en 2005. Sa raison
} 
Par ailleurs, l'association Adefis (Association d'expérimentation en formation interdisciplinaire et scientifique, siège à l'ENS, 45 rue d'Ulm, Paris) dont l'objet est la promotion et le soutien financier des approches interdisciplinaires lors des formations universitaires offre un lien entre les différentes générations d'étudiants qui ont suivi la formation $\mathrm{AIV}^{31}$.

Source $\quad$ http://www.master-aiv.fr/

http://www.univ-paris5.fr/spip.php?article1713

\section{Une maîtrise en sciences de l'environnement à l'université du Québec, Montréal (Laurent LEPAGE)}

\section{Intitulé de la formation (titre)}

Niveau (master 1, 2)

Master Pro ou recherche

Responsable(s)

Date de création

\begin{abstract}
Maîtrise en sciences de l'environnement, Université du Québec à Montréal
\end{abstract}

Masters 1 et 2

Profil avec stage en milieu professionnel

Profil recherche

Pierre DRAPEAU, Directeur du programme, professeur à l'Institut des sciences de l'environnement, et au département des sciences biologiques - UQAM

1973

\footnotetext{
d'être est d'offrir un lieu convivial d'échanges aux carrefours des sciences de la vie, des sciences exactes, sciences naturelles, cognitives et sociales Ce lieu comprend une salle de séminaire entièrement équipée, une salle de réunion, une salle d'échanges informels (salle café), des bureaux d'accueil destinés aux professeurs visiteurs, une bibliothèque, un laboratoire de modélisation ainsi qu'un laboratoire expérimental. Il accueille les cours du M 2 des clubs de discussion d'étudiants, dont le club de biologie synthétique qui a donné naissance à la première équipe française de biologie synthétique. Créé par des étudiants du master AIV, ils ont remporté le prix de la recherche fondamentale au concours iGEM organisé par le MIT devançant ainsi une cinquantaine d'équipes anglo-saxonnes travaillant sur ce sujet.

Le CRI accueille aussi les étudiants et les enseignants (dont des professeurs visiteurs prestigieux venant des plus grandes universités mondiales) de l'École doctorale interdisciplinaire européenne « Frontières du Vivant ». Enfin le CRI abrite la chaire de recherche de France Télécom dédié aux interfaces entre le vivant, la modélisation et les nouvelles technologies.

Une autre perspective d'avenir sera à partir de la rentrée 2009 la mise en place d'un master M 1 basé sur les mêmes principes mais avec plus de cours dont des approches de modélisation du vivant, des cours sur de nouvelles méthodologies expérimentales et de nouvelles approches de la modélisation et de l'analyse de données, des cours de biologie systémique, de biologie synthétique, de biologie intégrative et de médecine moléculaire.

${ }^{31}$ Elle offre également un cadre pour la réalisation de projets initiés par les étudiants. Ainsi, en avril 2008, 15 étudiant en M 2 ou en thèse rencontreront 15 homologues chinois à l'université de Pékin, pour 15 jours de création de projets de recherche et visite de laboratoire chinois. Depuis septembre 2007, Adéfis contribue à la mise en place d'un réseau européen d'associations étudiantes proposant des contenus pédagogiques au sein de universités (EAGER : European Association for a Greater Enthusiasm in Research). En février 2008, les workshops animés par les étudiants du CRI pourront être partagés par vidéoconférence avec des étudiants de l'université de Zagreb en Croatie.

Régulièrement en collaboration avec le CRI, Adéfis organise des Scicamp. Inauguré par Nature et Google aux États-Unis en 2006, le Scicamp est un lieu de débat et de discussion ouvert à tous. Les participants proposent les thématiques qui seront discutées en suivant. Rien n'est donc fixé à l'avance.
} 


\section{Historique}

Institution de rattachement

\section{Disciplines enseignées}

Objectif(s) de

l'enseignement

(compétences

interdisciplinaires

recherchées)
Ouverte initialement en 1973 sous le nom de «Maîtrise en écologie», cette formation traduisait les préoccupations environnementales de ce temps (les aménagements majeurs et leurs conséquences sur le milieu biophysique ainsi que la notion encore récente de pollution). Entre 1973 et 1987, la maîtrise évolue selon certaines caractéristiques :

- elle inclut à la fois des aspects axés sur la pratique (stages) et des aspects plus traditionnels de l'Université (recherche); - elle permet l'ouverture aux différentes disciplines qui constituent les sciences de l'environnement (pas uniquement la discipline de premier cycle des étudiants) mais elle vise principalement un approfondissement disciplinaire à travers des problématiques à connotation environnementale.

1987 : Réforme majeure du programme, dont l'introduction d'un choix entre deux profils : «professionnel» et «recherche» et des modifications aux cours.

1990 : Création de l'Institut des sciences de l'environnement auquel sont rattachés les programmes de maîtrise et de doctorat en sciences de l'environnement (ces programmes ne relevant d'aucun département). Quoique officiellement rattaché à la Faculté des sciences, l'Institut rassemble des professeurs provenant de toutes les facultés et plusieurs départements de l'Université.

1997 : Seconde réforme majeure :

- disparition des objectifs d'approfondissement disciplinaire au profit de l'interdisciplinarité ;

- changements dans la base d'admission et ouverture explicite aux diplômés de sciences humaines et sociales ;

- restructuration complète de la banque de cours (élimination des cours «disciplinaires» et «complémentaires») : création d'un tronc commun et d'un nouveau cursus complet de cours, basé non plus sur des disciplines mais intégrant l'interdisciplinarité.

Institut des sciences de l'environnement, Université du Québec à Montréal (UQAM)

Sciences naturelles, sciences sociales ou sciences humaines.

- Objectif général et fondamental : Comprendre et évaluer l'articulation entre la dynamique des systèmes naturels, les dynamiques économique, sociale, politique, culturelle et la santé humaine. Développer des compétences permettant de participer, par la mise en commun d'expertises disciplinaires, thématiques ou sectorielles, à la définition, la gestion et la résolution de problèmes environnementaux dans le but de contribuer à la mise en œuvre d'un développement durable.

- Objectif particulier au profil recherche : Développer des compétences de recherche interdisciplinaire sur des problématiques environnementales pouvant conduire à des études doctorales ou à une application sur le marché du travail.

- Objectif particulier au profil professionnel : Développer la capacité d'intervenir sur le marché du travail de manière innovatrice dans la définition, la gestion et la résolution de problèmes environnementaux. 


\section{Descriptif de \\ l'enseignement}

(cours, TP, terrain, etc.)

\section{Comment est organisée l'interdisciplinarité dans les enseignements ?}

\author{
Nombre moyen d'étudiants \\ (par années)
}

Origines disciplinaires des étudiants

\author{
Comment est prise \\ en charge \\ l'hétérogénéité \\ des étudiants?
}

\section{Existe-t-il des échanges entre étudiants à l'occasion de travaux collectifs?}

\section{Débouchés envisagés et/ou effectifs}

A. Tronc commun avec quatre activités :

1. Perspectives interdisciplinaires dans l'étude des problématiques environnementales : cette activité qui constitue la majeure partie du tronc commun du programme vise deux objectifs :

- la compréhension du rôle, de l'apport et des limites des différentes pratiques scientifiques dans l'analyse et la résolution de problèmes environnementaux ;

- la compréhension et l'analyse de la dynamique des systèmes naturels et de la dynamique du développement économique et social qui interviennent dans l'évolution des problématiques environnementales. Évolution des paradigmes en sciences de l'environnement. Aspects historiques et éthiques des enjeux environnementaux. Composantes bio-géophysiques des systèmes et leurs interactions. Principes d'analyse systémique, de développement durable et de gestion intégrée des ressources. Rôle des acteurs. Interactions multidisciplinaires. Exemples vécus par des équipes de recherche. Problématiques locales, régionales et globales. Possibilités de sorties sur le terrain.

2. Éléments d'épistémologie.

3. La méthode scientifique (hors programme).

4. Communication scientifique.

B. Cours de spécialisation avec stage pour le profil pro et mémoire pour le profil recherche.

L'enseignement est généralement dispensé sous forme de «Team teaching». Les équipes sont composées de deux ou trois professeurs provenant de disciplines différentes mais intervenant dans le cadre d'une même problématique environnementale.

Environ 40-50 étudiants sont admis à la maîtrise chaque année à la session d'automne. La durée prévue de leurs études est de deux ans (6 sessions).

Sciences naturelles, sciences humaines ou sociales.

Le tronc commun donné lors de la première session aborde les principaux concepts liés aux sciences de l'environnement et donne l'occasion aux étudiants de développer une plate-forme permettant les échanges interdisciplinaires. Le tronc commun utilise à la fois des cours magistraux (au sein du groupe élargi) et des ateliers en plus petits groupes de travail (comprenant des étudiants provenant de différentes disciplines). La " résolution de problème » s'avère le liant le plus utile.

Oui. Des travaux collectifs en équipe interdisciplinaire sont prévus dans la plupart des cours, que ce soit les cours du tronc commun ou les cours de spécialisation.

Nos diplômés travaillent dans divers milieux, soit gouvernementaux (au niveau fédéral, provincial ou municipal), para-gouvernementaux, communautaires, en enseignement ou en entreprise privée. 


\section{Nombre d'enseignants}

Statuts des enseignants

$Y$ a-t-il concertation entre enseignants? Sous quelle forme?

\section{Difficultés rencontrées}

Propositions, pistes pour des améliorations

Sources
Environ 50 (professeurs, professeurs associés, chargés de cours), cependant un premier cercle d'une dizaine d'enseignants est plus présent. À lui seul, le cours de « Perspectives interdisciplinaires » fait intervenir plus d'une vingtaine d'enseignants. Au niveau de l'enseignement, il y a aussi les autres cours de tronc commun et les cours de spécialisation. De plus, il faut considérer que plusieurs enseignants agissent comme directeurs de recherche et tuteurs (profil pro).

\section{Universitaires}

Comité de programme où siègent professeurs et étudiants Concertation dans le cadre du cours ENV7000 (Perspectives interdisciplinaires dans l'analyse des problématiques environnementales). Réunions de planification des cours de tronc commun auxquelles les professeurs intéressés sont conviés.

- Disponibilité des ressources professorales par rapport aux besoins d'encadrement (nombre élevé d'inscriptions) : nouvelles embauches par le programme de maîtrise ne sont pas possibles car il n'est pas rattaché à un département (les professeurs enseignant à la maîtrise proviennent de divers départements).

- Il y a risque de niveler vers le bas à cause de l'hétérogénéité du groupe, les formules pédagogiques sont donc a géométrie variable. - L'interdisciplinarité en environnement faisant appel tant aux sciences naturelles qu'aux sciences humaines ou sociales, des difficultés administratives découlent du rattachement du programme de maîtrise à une seule faculté.

- En ce qui a trait au financement de la recherche, les organismes subventionnaires de bourses reconnaissent très peu

l'interdisciplinarité ; leurs programmes sont divisés par domaines d'études, domaines souvent très conventionnels et disciplinaires. - De même, les mémoires de recherche interdisciplinaires peuvent être moins bien évalués ou compris par certains correcteurs parce que sortant des normes de leur discipline respective.

- Afin d'éviter de niveler par le bas lorsque les groupes d'étudiants ne sont pas homogènes, il peut être intéressant de s'arrimer à ce que les étudiants savent déjà afin de construire à partir de ce qu'ils ont déjà intégré.

- Sensibiliser les gestionnaires de l'université et les instances décisionnelles (administration, syndicats) aux particularités de l'interdisciplinarité afin que les normes administratives relatives à l'embauche de professeurs puissent être adaptées aux besoins de l'interdisciplinarité.

- Sensibiliser les organismes subventionnaires et les membres des comités d'évaluation des mémoires afin qu'ils comprennent mieux l'interdisciplinarité et considèrent de façon juste et équitable les projets (pour les bourses) et les mémoires (pour leur évaluation finale) des étudiants en sciences de l'environnement.

Notes et site $:$ http://www.ise.uqam.ca/maitrise/index.php Institut des sciences de l'environnement, UQAM (2003). Rapport sur l'évaluation du programme de maîtrise en sciences de l'environnement 1997-2001 


\title{
Annexes
}

\section{Argumentaire et programme des Journées}

\author{
LES FORMATIONS INTERDISCIPLINAIRES : \\ EXPÉRIENCES, PROBLÈMES, PERSPECTIVES \\ Mercredi 7 et jeudi 8 février 2007 \\ École normale supérieure, salle Dussane, 45 rue d'Ulm, Paris V
}

L'idée d'une formation impliquant l'apprentissage conjoint de plusieurs disciplines ne date pas d'aujourd'hui. Toutefois, elle connaît un regain d'intérêt, s'étend à de nouveaux domaines et suscite des innovations qui ont souvent du mal, du fait même de leur caractère novateur, à se construire, tant sur le plan de leur contenu que sur celui de leurs démarches de formation. Ce que nous vous proposons ici est une confrontation des expériences pour les clarifier, pour formuler les questions de fond qu'elles soulèvent et réfléchir en commun aux façons d'y répondre. Cette réflexion devrait permettre de mieux faire face aux difficultés que ces initiatives rencontrent. On ne se lance pas dans la conception et la mise en œuvre d'une formation interdisciplinaire sans raisons. Elle suppose l'explicitation et l'élaboration d'un projet de formation original qui est conçu en fonction de sa raison d'être et qui doit donc répondre à ses exigences. Elle est le résultat d'une réflexion nécessairement collective. Au-delà de cette réflexion, c'est dans son incarnation pratique que se joue l'essentiel. En effet, qu'il l'exprime ou non, le projet implique une conception de l'interdisciplinarité. Celle-ci est fonction des finalités poursuivies, mais renvoie aussi à une posture intellectuelle. Les disciplines associées, les méthodologies privilégiées, les modalités de la formation et de l'évaluation, etc. en sont l'expression. Le cursus peut se contenter de juxtaposer des enseignements disciplinaires donnés dans des formes tout à fait habituelles et, dans ce cas, on peut parler de "pluridisciplinarité ».

À l'opposé, il peut être délibérément construit autour d'une démarche visant à réfléchiret à faire réfléchir - sur le découpage disciplinaire qui est au cour du projet de formation et à trouver les voies d'un dialogue entre les disciplines concernées ; alors, on pourrait parler simultanément de "formation interdisciplinaire » et de "formation à l'interdisciplinarité ».

Mais les distinctions à faire sont sans doute plus subtiles. Elles dépendent en effet des disciplines associées, de leur rapport relatif, ainsi que des objets, des thèmes et des questions de recherche autour desquels la formation est conçue. Une polarisation forte des disciplines convoquées autour d'objets, de thèmes et de questions de recherche largement partagés peut produire une interdisciplinarité de fait que l'on pourrait qualifier d'implicite et elle est encore renforcée si la polarisation en question est par ailleurs associée à une longue expérience d'enseignement en commun. Dans d'autres cas la formation sur et par l'interdisciplinarité impliquera une relation plus réflexive entre les disciplines et les enseignants, et donc une véritable initiation à l'épistémologie. 
Mercredi 7 février 2007

8h30 Accueil

9h Présentation, ClaUde MiLlier, Président de NSS-Dialogues, INAPG et Engref, et Introduction, MARCEL JOLLIVET, CNRS

9h45 Les sciences cognitives : un champ de recherche en quête de son interdisciplinarité, DANIEL ANDLER, ENS

10h30 Archéologie et histoire : un dialogue ancien, DOMINIQUE MARGUERIE, paléo-environnementaliste et JEAN-LAURENT MONNIER, université Rennes 1 Président discutant : MARIE-Christine MARINVAL, université Paris 1

11h15 Écologie et sciences sociales, LUC ABBADIE, CNRS ET JACQUES WEBER, Institut français de la biodiversité

Président discutant : SERGE MORAND, Ensam, Inra

$12 \mathrm{~h} \quad$ Repas

14h Former à l'environnement : un chantier en cours, CATHERINE CARRE, université Paris 1 et Cheminements et questionnements sur l'interdisciplinarité et l'interculturel, ANNE-MARIE GRANIE, École nationale de formation agronomique de Toulouse

Président discutant : DANIEl DelahaYe, université de Caen-Basse-Normandie

14h45 Environnement et sociétés : l'expérience d'un master, MARIE ROUE, CNRS Président discutant : LAURA RIVAL, université d'Oxford, EHESS

$15 h 30$ Pause

$15 \mathrm{~h} 45 \quad$ Présentations d'expériences :

Modélisation : • Informatique et biologie, DANIEL AUCLAIR, Inra

- Mathématiques et sciences sociales, CECILIA CLAEYSMEKDADE, université de la Méditerranée

- Modélisation entre natures et sociétés, DOMINIQUE HERVE/FRANCIS LALOË, IRD

Environnement : • «Environnement, santé, société » : enjeux pédagogiques et scientifiques d'une formation, PATRICK GIRAUDOUX, université de Franche-Comté

- Un exemple canadien : l'Institut des sciences de l'environnement de l'UQAM, LAURENT LEPAGE, ISE, UQAM, Canada/ÉRIC DUCHEMIN, directeur de VertigO, ISE, UQAM, Canada

- Le master « Économie et gestion de l'environnement et du développement durable " : avenir d'une illusion ou illusion d'avenir? MURIEL MAILLEFERT, université de Lille 3 
Jeudi 8 février 2007

$9 \mathrm{~h}$

L'écologie humaine : carrefour de disciplines et représentations transdisciplinaires, FRANCIS RIBEYRE, université de Bordeaux Président discutant : BERNARD BRUN, Société d'écologie humaine

$9 \mathrm{~h} 45$ Mélange des cultures et pédagogie de projet. Réflexions sur la formation des ingénieurs des Eaux et des Forêts, JACQUES BREGER, Engref Président discutant : Nicolas BoulEaU, École des Ponts et Chaussée

$10 h 30$ Pause

10h45 Approche interdisciplinaire du vivant, FRANÇOIS TADDEÏ, ENS Président discutant : ANNICK LESNE, CNRS

$12 \mathrm{~h} \quad$ Repas

14h Table ronde : Le point de vue des jeunes diplômés interdisciplinaires, animée par NICOLE MATHIEU

- Sciences cognitives : Élise Mettereau et François-Xavier Penicaud, représentants de Fresco (Fédération française des étudiants et jeunes chercheurs en sciences cognitives)

- Sciences sociales : JUHANE DASCON ET REMI BENOS, représentants d'Essor (Espaces, sociétés rurales et logiques économiques), Toulouse

- Sciences de l'environnement : FRANÇOISE BENEVISE, RICHARD DUMEZ et DELPHINE GRAMOND, représentants de Com'etes (assoc. des anciens étudiants du DEA ETES, Environnement : temps, espaces, sociétés).

- Sciences humaines : ALICE BENOIT, présidente d'Écosens, Association des étudiants et des diplômés du master de psychologie environnementale de Paris V)

$15 h 15 \quad$ Pause

15h30 Table ronde : Problèmes, contraintes et pistes, Président : ÉRIC VINDIMIAN, chef du service Recherche et Prospective, ministère de l'Environnement et du Développement durable avec HenRi BUC, CNRS/Institut Pasteur ; JeAn-PaUl Deleage, université d'Orléans ; LAURENT LEPAGE, université du Québec à Montréal ; JEAN-LOUIS MARTINAND, ENS Cachan

16h45 Bilan critique des Journées

$17 \mathrm{~h} \quad$ Clôture des Journées 


\section{Textes sur la formation parus dans la revue Natures Sciences Sociétés}

«L'écologie à l'école. Inventer un avenir pour la planète », Yves Bertrans, Paul Valois et France Jutras, Natures Sciences Sociétés, 1997, vol. 5, n³ 3, p. 82.

«Formation et environnement Les métiers de l'environnement. De nouvelles formations pour un avenir vert », Natures Sciences Sociétés, 1999, vol. 7, n 4, p. 65.

«Pourquoi étudier la vulgarisation des sciences forestières? Études des formations à la gestion des forêts », Pascal Marty, Natures Sciences Sociétés, 2000, vol. 8, n 1, p. 63-65.

"Compte-rendu de colloque: 'Les case studies, une approche spécifique pour la formation en environnement des universitaires et des ingénieurs' », Magda Zanoni, Natures Sciences Sociétés, 2000, vol. $8, \mathrm{n}^{\circ} 1$, p. 66-68.

«L'interdisciplinarité vue et pratiquée par les chercheurs en Sciences de la vie », Jean-Marie Legay, la table ronde des journées NSS 2002, Natures Sciences Sociétés, vol. 12, n 1, 2004, p. 63-74.

«Jeunes chercheurs doctorants : professionnaliser, mais aussi (ré)former », Jean-Paul Billaud, Henri Décamps et Bernard Hubert, Natures Sciences Sociétés, 2004, vol. 12, n 4, p. 373-374.

«Cécilia Claeys-Mekdade, une sociologue face à l'interdisciplinarité : éloge du doute méthodologique. Propos recueillis par Agnès Pivot ", Cécilia Claeys-Mekdade et Agnès Pivot, Natures Sciences Sociétés, 2005, vol. 13, n² 2 p. 189-193.

«Une expérience de formation interdisciplinaire aux recherches sur le développement durable : la chaire Unesco de l'université fédérale du Paraná (Curitiba, Brésil)», Magda Zanoni, Claude Raynaut et Francisco Mendonça, Natures Sciences Sociétés, 2005, vol. 13, n² 2, p. 198-205.

«Dossier Interdisciplinarité. Évaluer les pratiques interdisciplinaires », Frédéric Joulian, Suzanne de Cheveigné et Joëlle Le Marec, Natures Sciences Sociétés, 2005, vol. 13, n 3, p. 284-290.

"Interdisciplinarity and young rural researchers », Dossier Interdisciplinarité, Ella Mustakangas, Petra Derkzen, Mélanie Gambino and Eva Kučerová, Natures Sciences Sociétés, 2006, vol. 14, $\mathrm{n}^{\circ} 2$, p. $179-184$.

«Interdisciplinarité et production de connaissances : quelles recherches voulons-nous ? \Jean-Paul Billaud, Bernard Hubert, Natures Sciences Sociétés, 2006, vol. 14, n 3, p. 233-234.

«Dossier Interdisciplinarité. Le jeune chercheur et l'interdisciplinarité en sciences sociales. Des pratiques remises en question », Ève Anne Bühler, Fabienne Cavaillé et Mélanie Gambino, Natures Sciences Sociétés, 2006, vol. 14, n 4, p. 392-398.

«L'institutionnalisation de l'interdisciplinarité au sein de l'université brésilienne: le cas de l'environnement », Marcel Burzstyn, Natures Sciences Sociétés, 2007, vol. 15, n 1, p. 57-64.

«Agnès Pivot: l'écriture, processus heuristique et outil pour le dialogue dans une démarche interdisciplinaire », Agnès Pivot, Nicole Mathieu, Natures Sciences Sociétés, 2007, vol. 15, n 4, p. 411-416.

«Environnement et interdisciplinarité : paradoxes dans le champ éducatif», Jean-Louis Martinand, Natures Sciences Sociétés, 2008, vol. 16, n 1, p. 1-2.

«Environnement et disciplines scolaires », Christine Vergnolle Mainar, Natures Sciences Sociétés, 2008 , vol. $16, \mathrm{n}^{\circ} 1$, p. $60-66$. 